\title{
HDDA-enabled carbazolyne chemistry: Single step, de novo construction of the pyranocarbazole core of alkaloids of the Murraya koenigii (curry tree) family
}

\author{
Tao Wang and Thomas R. Hoye* \\ Department of Chemistry, University of Minnesota, 207 Pleasant St. SE, Minneapolis, MN 55455 \\ E-mail: hoye@umn.edu
}

\section{Table of Contents}

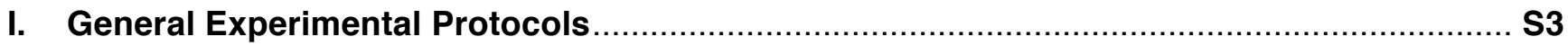

II. Preparation and characterization data for all key compounds ....................................... S4

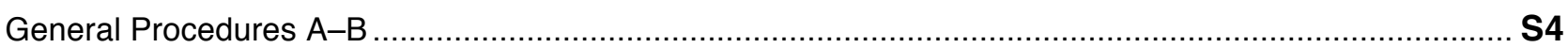

Preparation of the multi-yne precursors for HDDA cycloisomerization ................................................S5

Preparation of carbazole derivatives from HDDA cycloisomerization reaction ......................................S13

III. Computational methods and coordinates of structures ............................................... S22

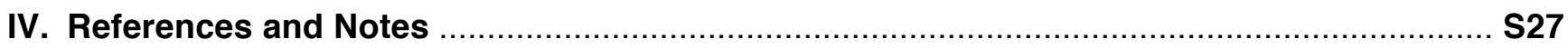

V. Copies of NMR spectra

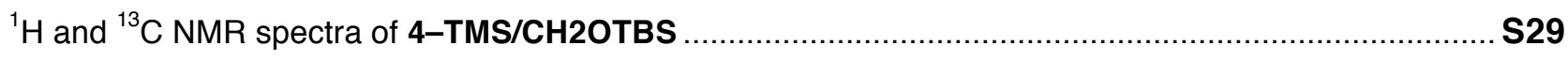

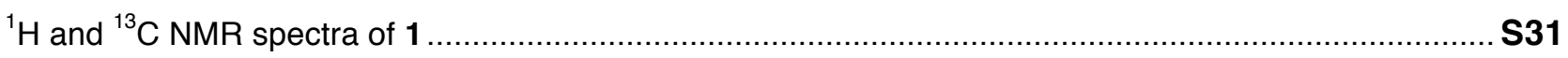

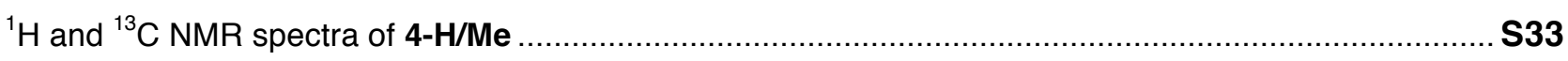

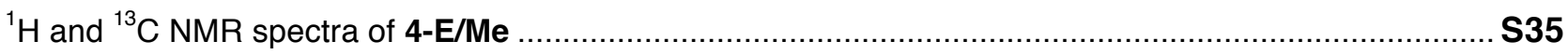

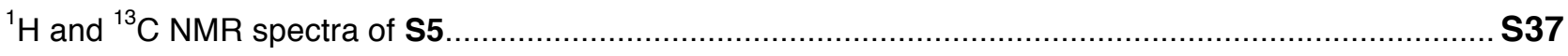

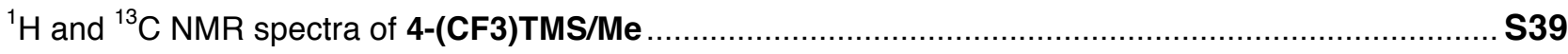

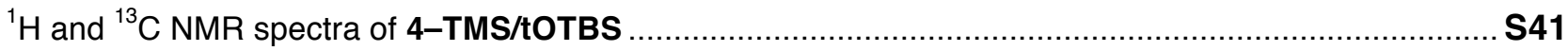

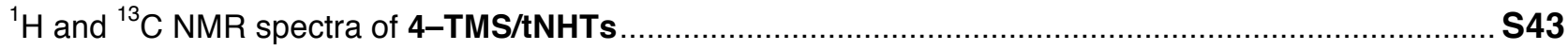

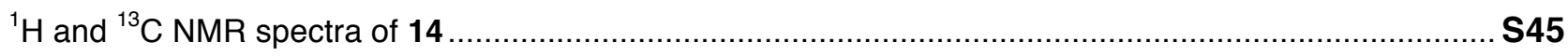

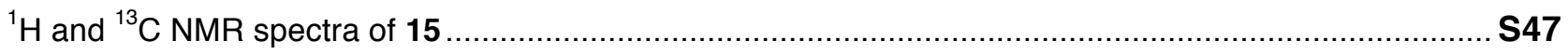

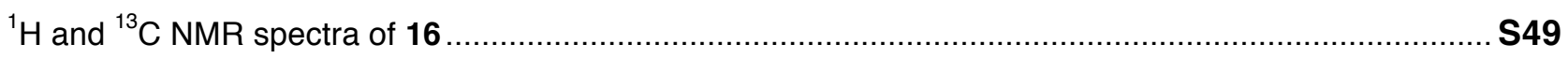

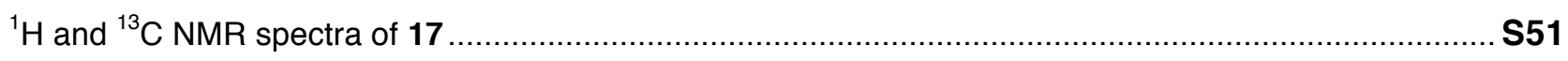

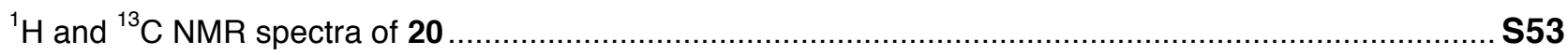




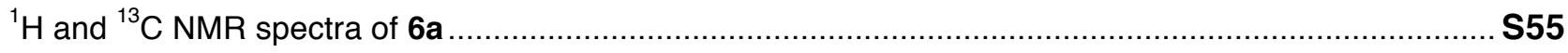

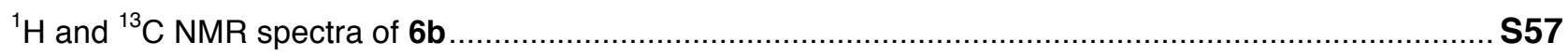

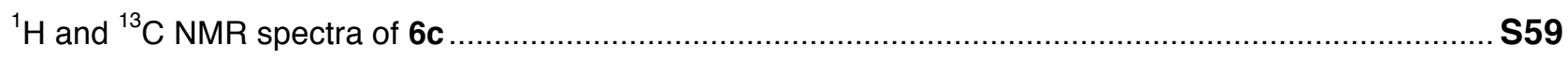

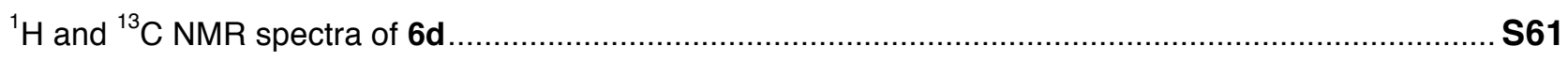

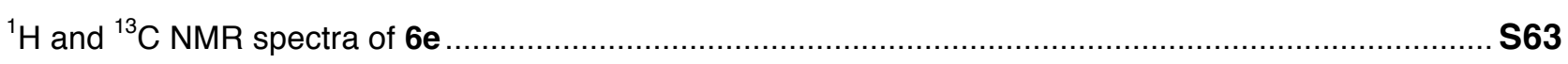

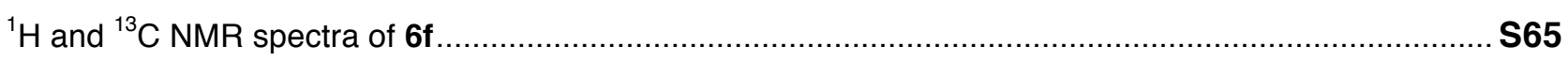

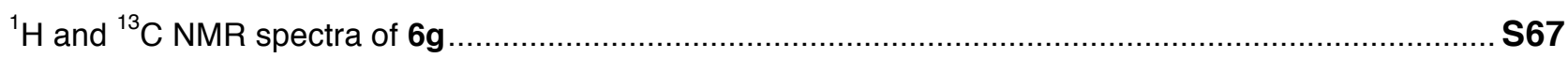

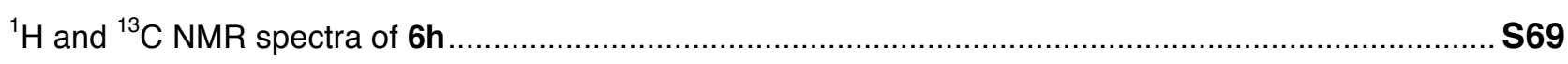

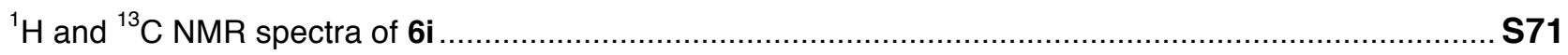

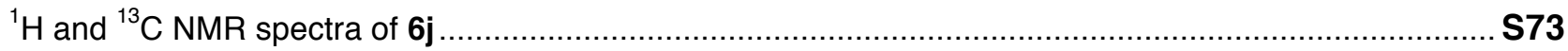

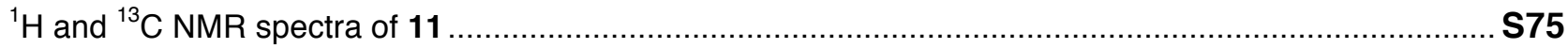

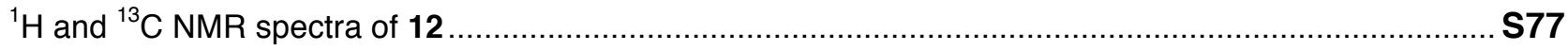

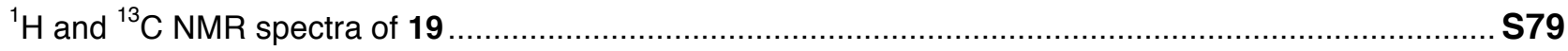

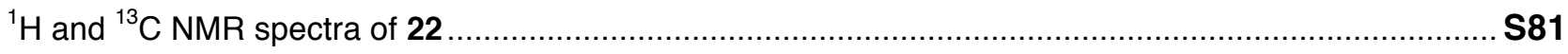




\section{General Experimental Protocols}

NMR spectra were recorded on either a Bruker Avance 400 or 500 (400 and $500 \mathrm{MHz}$, respectively) spectrometer in $\mathrm{CDCl}_{3}$. The following format is used to report the ${ }^{1} \mathrm{H}$ NMR data: chemical shift in ppm [multiplicity, coupling constant(s) in Hz, integral (to the nearest whole number of protons), and assignment]. Coupling constant analysis was guided by methods we have previously reported. ${ }^{1}$ Chemical shifts for proton spectra are referenced to TMS $(\delta 0.00 \mathrm{ppm})$. Non-first order multiplets are listed as "nfom". Chemical shifts for carbon spectra are referenced to $\mathrm{CHCl}_{3}(\delta 77.23 \mathrm{ppm})$. TMS is detectable in some of the ${ }^{13} \mathrm{C}$ NMR samples $(\delta$ ca. $0.0 \mathrm{ppm})$. Several compounds [4-(CF3)TMS/Me, $\mathbf{6 h}, \mathbf{2 0}$, and 22] contain $\mathrm{CF}_{3}$ groups. The resonances for several carbon atoms in the ${ }^{13} \mathrm{C}$ NMR spectra for these are observed as quartets; for each, the midpoint of the multiplet (i.e., the actual chemical shift) is given in the line listing.

Infrared spectral data were recorded on an Midac Corporation Prospect 4000 FT-IR spectrometer. Spectra were taken of thin film samples in attenuated total reflectance (ATR) mode on a germanium window.

Electrospray ionization (ESI) mass spectrometry data were collected on a Bruker BioTOF II (ESI-TOF) instrument. Samples for high-resolution mass spectral (HRMS) analysis were doped with PEG as the internal calibrant. Samples were introduced as methanolic solutions.

Flash chromatography was performed on E. Merck silica gel (230-400 mesh). Thin layer chromatography (TLC) was performed on glass or plastic backed plates of silica gel. Spots were visualized by dipping in a solution of ceric ammonium molybdate followed by heat treatment and/or UV irradiation.

Reactions requiring anhydrous conditions were performed in flame- or oven-dried glassware under an atmosphere of nitrogen or argon. Anhydrous toluene, diethyl ether, THF, and methylene chloride were passed through a column of activated alumina and taken immediately prior to use. $\mathrm{CHCl}_{3}$ used for HDDA reactions was ethanol-free. Reported reaction temperatures are the temperature of the external heating oil-bath. HDDAinitiated reactions, including those that were carried out at temperatures above the boiling point of the solvent, were typically performed in a threaded vial fitted with an inert, Teflon ${ }^{\circledR}$-lined screw-cap.

Procedures for preparation and spectral data are provided for i) all new compounds specifically shown in the manuscript and ii) all new (previously unreported) intermediates used in the synthetic route by which the former

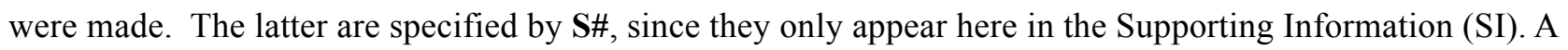
citation is provided for each known but non-commercially available compound that is used; these have not been given a structure number. 


\section{Preparation and characterization data for all key compounds}

\section{General Procedures A-B}

\section{A. Alkyne coupling with the Cadiot-Chodkiewicz reaction}

To a solution of $\mathrm{CuCl}(0.10$ equiv or $10 \%$ with respect to (wrt) the terminal alkyne substrate) in 40:60 (v:v) $n$ $\mathrm{BuNH}_{2}: \mathrm{H}_{2} \mathrm{O}(5 \mathrm{~mL} / \mathrm{mmol})$ was added an excess of $\mathrm{NH}_{2} \mathrm{OH} \cdot \mathrm{HCl}$ (typically a few crystals on a reaction scale $\leq 1$ $\mathrm{mmol}$ ) with stirring. The reaction vessel was immediately capped with a Teflon ${ }^{\circledR}$-lined cap and placed in an icewater bath. The color of this mixture turned from deep purple-blue to colorless within seconds, indicating full reduction of $\mathrm{Cu}(\mathrm{II})$ to $\mathrm{Cu}(\mathrm{I})$. A mixed solution of the terminal alkyne (1.0 equiv) and the 1-bromoalkyne (1.21.5 equiv) in $\mathrm{CH}_{2} \mathrm{Cl}_{2}(5 \mathrm{~mL} / \mathrm{mmol}$ wrt the terminal alkyne) was added dropwise. The mixture was stirred for $1 \mathrm{~h}$ at $0{ }^{\circ} \mathrm{C}$, during which time a few crystals of $\mathrm{NH}_{2} \mathrm{OH} \cdot \mathrm{HCl}$ were periodically added whenever the solution turned bluish. After complete consumption of the terminal alkyne substrate (as monitored by TLC analysis), the mixture was diluted with saturated aqueous $\mathrm{NH}_{4} \mathrm{Cl}$ and extracted with EtOAc. The organic extracts were washed with brine, dried $\left(\mathrm{Na}_{2} \mathrm{SO}_{4}\right)$, filtered, and concentrated. The residue was typically purified by flash chromatography on silica gel.

\section{B. Carbazole synthesis with the HDDA reaction}

The indicated triyne (or tetrayne) precursor was added to a glass vial. 1,2-Dichloroethane (DCE) or chloroform was added to bring the concentration of the multi-yne to $0.02 \mathrm{M}$. The indicated trapping agent ( $2-10$ equiv) was then added. The vial was sealed with a Teflon-lined cap and placed in an oil bath held at 90,100 , or $120^{\circ} \mathrm{C}$.

After $15 \mathrm{~h}$, the reaction vessel was allowed to cool to room temperature, concentrated, and directly subjected to flash column chromatography on silica gel for purification. 
Preparation of the multi-yne precursors (i.e., 1, 4, 17, and 20) for HDDA cycloisomerization
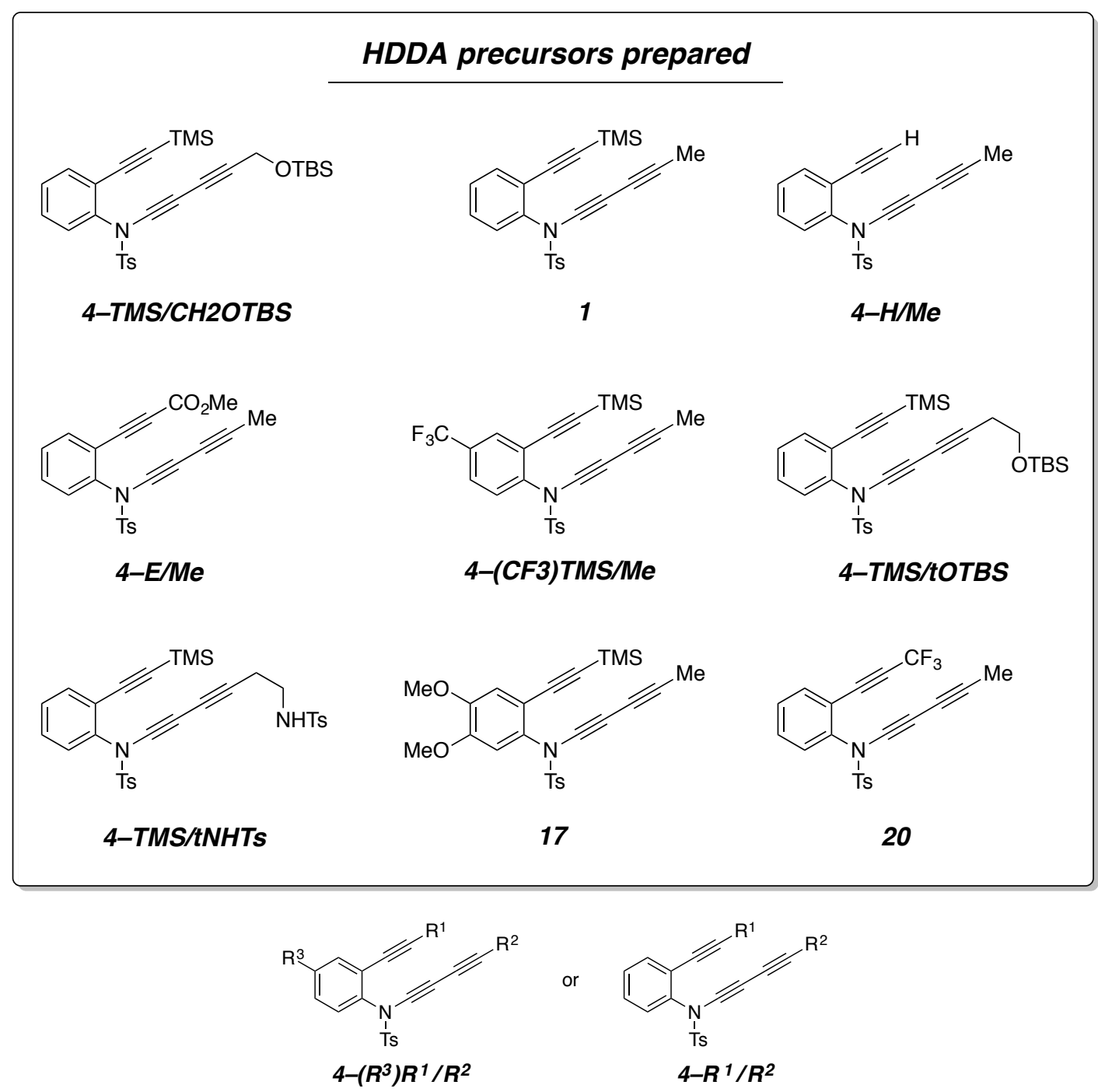

Naming logic for substrates 4: 4-( $\left.\mathbf{R}^{3}\right) \mathbf{R}^{\mathbf{1}} / \mathbf{R}^{\mathbf{2}}$ or $\mathbf{4}-\mathbf{R}^{\mathbf{1}} / \mathbf{R}^{\mathbf{2}}$ is used for HDDA precursors with the following structure unless otherwise defined. For any substrate with an intramolecularly tethered trapping group $R$, " $t R$ " is used in lieu of $\mathrm{R}^{2}$ (e.g., "tOTBS" signifies that an OTBS group is tethered as the intramolecular trapping agent).

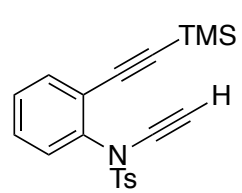

S1

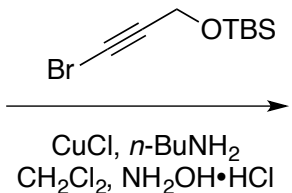

$\mathrm{CH}_{2} \mathrm{Cl}_{2}, \mathrm{NH}_{2} \mathrm{OH} \cdot \mathrm{HCl}$

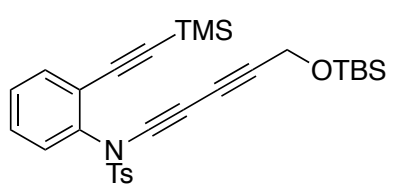

4-TMS/CH2OTBS

Preparation of $N$-(5-((tert-butyldimethylsilyl)oxy)penta-1,3-diyn-1-yl)-4-methyl- $N$-(2((trimethylsilyl)ethynyl)phenyl)benzenesulfonamide (4-TMS/CH2OTBS)

Triyne 4-TMS/CH2OTBS was prepared following General Procedure A using diyne precursor $N$-ethynyl-4methyl- $N$-(2-((trimethylsilyl)ethynyl)phenyl)benzenesulfonamide ${ }^{2}$ (43 mg, $\left.0.12 \mathrm{mmol}\right)$, ((3-bromoprop-2-yn-1yl)oxy)(tert-butyl)dimethylsilane $(50 \mu \mathrm{L}), \mathrm{CuCl}(1 \mathrm{mg}, 0.01 \mathrm{mmol}), n-\mathrm{BuNH}_{2}(0.5 \mathrm{~mL})$, and $\mathrm{CH}_{2} \mathrm{Cl}_{2}(0.5 \mathrm{~mL})$. Purification by flash column chromatography (hexanes: ethyl acetate $=5: 1$ ) gave the triyne 4-TMS/CH2OTBS (46.1 mg, $0.086 \mathrm{mmol}, 74 \%$ ) as a pale yellow oil. 
${ }^{1}$ H NMR (500 MHz, $\mathrm{CDCl}_{3}$ ): $\delta 7.68\left(\mathrm{~d}, J=8.3 \mathrm{~Hz}, 2 \mathrm{H}, \mathrm{SO}_{2} \mathrm{Ar} H_{\text {ortho }}\right), 7.48-7.45$ (nfom, $\left.1 \mathrm{H}, \mathrm{ArH}\right), 7.35-7.28$ (nfom, 5H, ArH), 4.40 (s, 2H, CH$\left.H_{2} \mathrm{OTBS}\right), 2.46$ (s, 3H, SO $\left.\mathrm{Ar}_{2} \mathrm{CH}_{3}\right), 0.89$ (s, 9H, Si $\left.\left(\mathrm{CH}_{3}\right) \mathrm{C}\left(\mathrm{CH}_{3}\right)_{3}\right), 0.16$ (s, 9H, $\left.\mathrm{Si}\left(\mathrm{CH}_{3}\right)_{3}\right)$, and $0.10\left(\mathrm{~s}, 6 \mathrm{H}, \mathrm{Si}\left(\mathrm{CH}_{3}\right) \mathrm{C}\left(\mathrm{CH}_{3}\right)_{3}\right)$.

${ }^{13}$ C NMR (125 MHz, $\left.\mathrm{CDCl}_{3}\right): \delta 145.3,138.4,134.1,130.0,129.32,129.30,129.2,128.7,127.4,122.7,102.2$, $99.4,81.9,70.1,69.6,58.6,52.5,26.0,22.0,18.5,-0.1$, and -5.0 .

IR: $2955,2929,2857,2246,2166,1483,1445,1380,1250,1175,1089,1023$, and $842 \mathrm{~cm}^{-1}$.

HRMS (ESI-TOF): Calcd for $\mathrm{C}_{29} \mathrm{H}_{37} \mathrm{NNaO}_{3} \mathrm{SSi}_{2}{ }^{+}[\mathrm{M}+\mathrm{Na}]^{+}$requires 558.1925; found 558.1931.

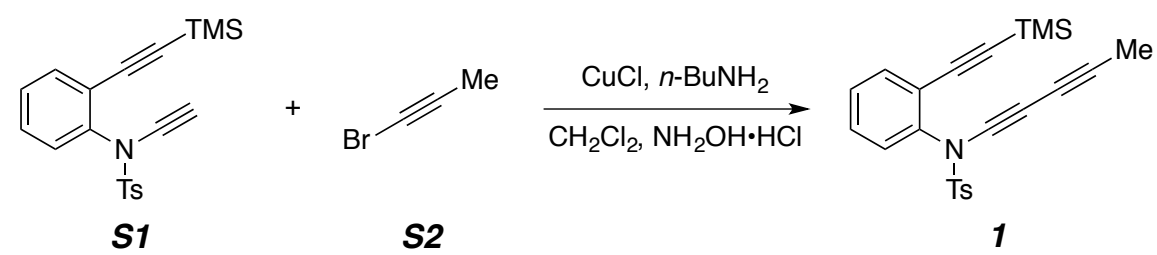

Preparation of 4-methyl- $N$-(penta-1,3-diyn-1-yl)- $N$-(2-((trimethylsilyl)ethynyl)phenyl)benzenesulfonamide (1)

Triyne 1 was prepared following General Procedure A using the diyne precursor $N$-ethynyl-4-methyl- $N$-(2((trimethylsilyl)ethynyl)phenyl)benzenesulfonamide ${ }^{3}(\mathbf{S 1}, 201 \mathrm{mg}, 0.55 \mathrm{mmol}), 1$-bromopropyne ${ }^{4}(\mathbf{S 2}, 0.8 \mathrm{~mL}$, 1.4 $\mathrm{M}$ in hexanes), $\mathrm{CuCl}(6 \mathrm{mg}, 0.06 \mathrm{mmol}), n-\mathrm{BuNH}_{2}(2 \mathrm{~mL})$, and $\mathrm{CH}_{2} \mathrm{Cl}_{2}(2 \mathrm{~mL})$. Purification by flash column chromatography (hexanes: ethyl acetate 12:1 to 5:1) gave the triyne $1(193 \mathrm{mg}, 0.48 \mathrm{mmol}, 87 \%)$ as a pale yellow oil.

${ }^{1}$ H NMR $\left(500 \mathrm{MHz}, \mathrm{CDCl}_{3}\right): \delta 7.67\left(\mathrm{~d}, J=8.3 \mathrm{~Hz}, 2 \mathrm{H}, \mathrm{SO}_{2} \mathrm{Ar} H_{\text {ortho }}\right), 7.48-7.44$ (nfom, $\left.1 \mathrm{H}, \mathrm{ArH}\right), 7.34-7.27$ (nfom, 5H, $\mathrm{ArH}), 2.45\left(\mathrm{~s}, 3 \mathrm{H}, \mathrm{SO}_{2} \mathrm{Ar}-\mathrm{CH}_{3}\right), 1.94\left(\mathrm{~s}, 3 \mathrm{H}, \mathrm{C} \equiv \mathrm{C}-\mathrm{CH}_{3}\right)$, and $0.17\left(\mathrm{~s}, 9 \mathrm{H},-\mathrm{Si}\left(\mathrm{CH}_{3}\right)_{3}\right)$.

${ }^{13}$ C NMR (125 MHz, $\left.\mathrm{CDCl}_{3}\right): \delta 145.1,138.7,134.7,134.2,130.0$ (br, 2C), 129.3, 129.1, 128.7, 122.7, 102.2, $99.6,80.4,66.4,64.2,59.1,22.0,4.8$, and 0.2 .

IR: $2958,2257,2172,1596,1483,1445,1379,1174,857$, and $845 \mathrm{~cm}^{-1}$.

HRMS (ESI-TOF): Calcd for $\mathrm{C}_{23} \mathrm{H}_{23} \mathrm{NNaO}_{2} \mathrm{SSi}^{+}[\mathrm{M}+\mathrm{Na}]^{+}$requires 428.1111; found 428.1107.

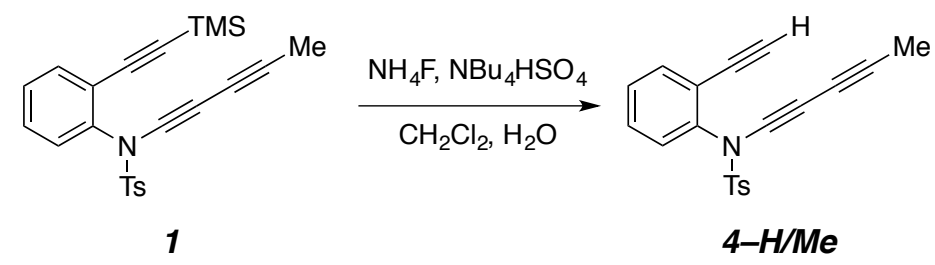

Preparation of $\mathrm{N}$-(2-ethynylphenyl)-4-methyl- $\mathrm{N}$-(penta-1,3-diyn-1-yl)benzenesulfonamide (4-H/Me)

Triyne 1 (30 mg, $0.074 \mathrm{mmol}), \mathrm{CH}_{2} \mathrm{Cl}_{2}(1 \mathrm{~mL})$, and $\mathrm{NBu}_{4} \mathrm{HSO}_{4}(2 \mathrm{mg}, 5.9 \mu \mathrm{mol})$ were combined in a small glass vial. A stir bar was added. $\mathrm{NH}_{4} \mathrm{~F}(\mathrm{aq}, 10 \mathrm{M})$ was added to the suspension at room temperature. The reaction mixture was stirred vigorously at room temperature for $2 \mathrm{~h}$. The mixture was partitioned between $\mathrm{NH}_{4} \mathrm{Cl}$ (aq) and ethyl acetate. The organic layer was washed with brine, dried with anhydrous $\mathrm{Na}_{2} \mathrm{SO}_{4}$, concentrated, and purified by column chromatography (hexanes: ethyl acetate $=5: 1)$ to give $\mathbf{4}-\mathbf{H} / \mathbf{M e}(15 \mathrm{mg}$, $0.045 \mathrm{mmol}, 61 \%$ ) as a pale yellow oil.

${ }^{1}$ H NMR $\left(500 \mathrm{MHz}, \mathrm{CDCl}_{3}\right): \delta 7.71\left(\mathrm{~d}, J=8.3 \mathrm{~Hz}, 2 \mathrm{H}, \mathrm{SO}_{2} \mathrm{Ar} H_{\text {ortho }}\right)$, 7.52-7.48 (nfom, 1H, ArH), 7.37-7.33 (nfom, 2H, $\mathrm{Ar} H$ ), $7.33\left(\mathrm{~d}, J=8.1 \mathrm{~Hz}, 2 \mathrm{H}, \mathrm{ArSO}_{2} \mathrm{Ar} H_{\text {meta }}\right.$ ), 7.22-7.18 (m, 1H, $\left.\mathrm{Ar} H\right), 3.03(\mathrm{~s}, 1 \mathrm{H}, \mathrm{C} \equiv \mathrm{CH}), 2.46$ (s, $\left.3 \mathrm{H}, \mathrm{SO}_{2} \mathrm{Ar}-\mathrm{CH}_{3}\right)$, and $1.95\left(\mathrm{~s}, 3 \mathrm{H}, \mathrm{C} \equiv \mathrm{C}-\mathrm{CH}_{3}\right)$. 
${ }^{13}$ C NMR $\left(125 \mathrm{MHz}, \mathrm{CDCl}_{3}\right): \delta 145.4,139.3,134.32,134.27,129.9,129.8,129.4,129.2,128.7,122.3,83.5$, $80.5,78.6,66.7,63.9,58.5,21.9$, and 4.9.

IR: $3283,2923,2256,2173,1482,1445,1375,1172,1089$, and $918 \mathrm{~cm}^{-1}$.

HRMS (ESI-TOF): Calcd for $\mathrm{C}_{20} \mathrm{H}_{15} \mathrm{NNaO}_{2} \mathrm{~S}^{+}[\mathrm{M}+\mathrm{Na}]^{+}$requires 356.0716; found 356.0719.

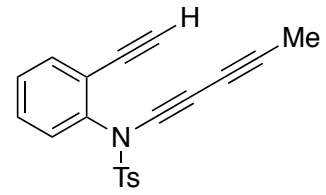

4-H/Me

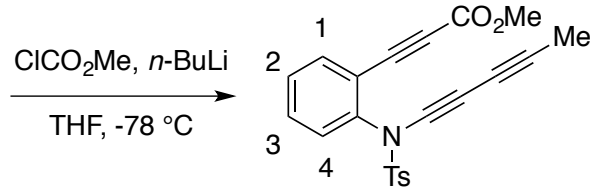

4-E/Me

Preparation of methyl 3-(2-((4-methyl- $N$-(penta-1,3-diyn-1-yl)phenyl)sulfonamido)phenyl)propiolate (4E/Me)

A solution of $N$-(2-ethynylphenyl)-4-methyl- $N$-(penta-1,3-diyn-1-yl)benzenesulfonamide (4-H/Me, $40 \mathrm{mg}, 0.12$ mmol) in THF $(2 \mathrm{~mL})$ was cooled to $-78{ }^{\circ} \mathrm{C}$ inside of a septum-sealed vial under a slight positive pressure of $\mathrm{N}_{2}$. A solution of $n$ - BuLi $(0.07 \mathrm{~mL}, 0.18 \mathrm{mmol}, 2.5 \mathrm{M}$ in hexanes $)$ was added dropwise via a syringe. This mixture was stirred at $-78{ }^{\circ} \mathrm{C}$ for $2 \mathrm{~h}$, and methyl chloroformate $(30 \mu \mathrm{L}, 0.39 \mathrm{mmol})$ was added. The vial was allowed to warm to room temperature over ca. $30 \mathrm{~min}$. The reaction mixture was partitioned between ethyl acetate and saturated aqueous solution of $\mathrm{NH}_{4} \mathrm{Cl}$. The organic layer was washed with brine, dried with anhydrous $\mathrm{Na}_{2} \mathrm{SO}_{4}$, filtered, and concentrated. The residue was purified by column chromatography on silica gel (hexanes:ethyl acetate $=5: 1)$ to give methyl 3-(2-((4-methyl- $N$-(penta-1,3-diyn-1-yl)phenyl)sulfonamido)phenyl)propiolate (4E/Me, $38 \mathrm{mg}, 0.1 \mathrm{mmol}, 81 \%$ yield $)$ as a yellow oil.

${ }^{1} \mathbf{H}$ NMR (500 MHz, $\mathrm{CDCl}_{3}$ ): $\delta 7.69\left(\mathrm{~d}, J=8.3 \mathrm{~Hz}, 2 \mathrm{H}, \mathrm{SO}_{2} \mathrm{Ar} H_{\text {ortho }}\right), 7.55$ (br dd, $\left.J=7.6,1.5 \mathrm{~Hz}, 1 \mathrm{H}, \mathrm{Ar} H_{1}\right)$, 7.47 (br ddd, $J=1.6,7.7,7.8 \mathrm{~Hz}, 1 \mathrm{H}, \mathrm{Ar} H_{3}$ ), 7.39 (br ddd, $J=1.1,7.6,7.6 \mathrm{~Hz}, 1 \mathrm{H}, \mathrm{ArH}_{2}$ ), 7.36 (br dd, $J=8.0$, $\left.0.8 \mathrm{~Hz}, 1 \mathrm{H}, \mathrm{Ar} H_{4}\right), 7.30$ (d, $\left.J=8.0 \mathrm{~Hz}, 2 \mathrm{H}, \mathrm{ArSO}_{2} \mathrm{ArH}_{\text {meta }}\right), 3.81\left(\mathrm{~s}, 3 \mathrm{H}, \mathrm{CO}_{2} \mathrm{CH}_{3}\right), 2.44\left(\mathrm{~s}, 3 \mathrm{H}, \mathrm{SO}_{2} \mathrm{Ar}-\mathrm{CH}_{3}\right)$, and $1.95\left(\mathrm{~s}, 3 \mathrm{H}, \mathrm{C} \equiv \mathrm{C}-\mathrm{CH}_{3}\right)$.

${ }^{13}$ C NMR $\left(125 \mathrm{MHz}, \mathrm{CDCl}_{3}\right): \delta 153.9,145.7,139.8,135.0,133.6,131.6,130.0,129.9,129.5,128.7,119.7$, $85.4,81.1,80.6,66.2,63.8,59.2,52.9,21.9$, and 4.8 .

IR: 2950 (w), 2900 (w), 2229, 2173, 1714, 1596, 1486, 1446, 1378, 1301, 1210, 1174, 1089, and $1029 \mathrm{~cm}^{-1}$.

HRMS (ESI-TOF): Calcd for $\mathrm{C}_{22} \mathrm{H}_{17} \mathrm{NNaO}_{4} \mathrm{~S}^{+}[\mathrm{M}+\mathrm{Na}]^{+}$requires 414.0770; found 414.0783.

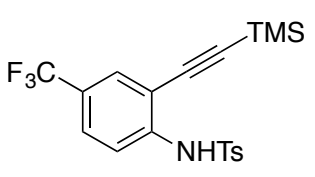

S3

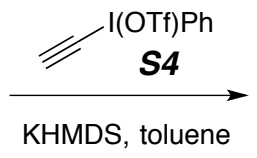

KHMDS, toluene

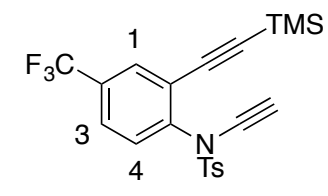

S5

Preparation of $\mathrm{N}$-ethynyl-4-methyl- $\mathrm{N}$-(4-(trifluoromethyl)-2-((trimethylsilyl)ethynyl)phenyl)benzenesulfonamide (S5)

A solution of amide $\mathbf{S 3}^{5}$ (105 mg, $0.26 \mathrm{mmol}$ ) in anhydrous toluene $(13 \mathrm{~mL})$ was added to a $100 \mathrm{~mL}$ round bottom flask. The reaction flask was cooled to $0{ }^{\circ} \mathrm{C}$ in an ice-water bath under $\mathrm{N}_{2}$ protection. A solution of KHMDS $(0.8 \mathrm{~mL}, 0.5 \mathrm{M}$ in toluene, $0.4 \mathrm{mmol})$ was added dropwise. After $45 \mathrm{~min}$, alkynyliodonium salt $\mathbf{S} 4^{6}$ (200 mg, $0.53 \mathrm{mmol}$ ) was added as a slurry in $\mathrm{CH}_{2} \mathrm{Cl}_{2}(25 \mathrm{~mL})$. After overnight stirring at room temperature, the reaction mixture was concentrated and partitioned between ethyl acetate and brine. The organic layer was dried with anhydrous $\mathrm{Na}_{2} \mathrm{SO}_{4}$, filtered, and concentrated. The residue was purified with column chromatography 
(hexanes: ethyl acetate $=12: 1$ to $5: 1)$ to give $\mathbf{S 5}(82 \mathrm{mg}, 0.19,74 \%)$ as a pale yellow oil.

${ }^{1} \mathbf{H}$ NMR $\left(500 \mathrm{MHz}, \mathrm{CDCl}_{3}\right): \delta 7.75\left(\mathrm{ddq}, J=2.2,0.5,0.7 \mathrm{~Hz}, 1 \mathrm{H}, \mathrm{Ar} H_{1}\right), 7.70(\mathrm{~d}, J=8.3 \mathrm{~Hz}, 2 \mathrm{H}$, $\left.\mathrm{ArSO}_{2} \mathrm{Ar}_{\text {ortho }}\right), 7.57$ (ddq, $\left.\left.J=8.4,2.2,0.7 \mathrm{~Hz}, 1 \mathrm{H}, \mathrm{ArH}\right)_{3}\right), 7.44$ (ddq, $\left.J=8.4,0.5,0.8 \mathrm{~Hz}, 1 \mathrm{H}, \mathrm{Ar} H_{4}\right), 7.33(\mathrm{~d}, J$ $\left.=8.1 \mathrm{~Hz}, 2 \mathrm{H}, \mathrm{ArSO}_{2} \mathrm{Ar} H_{\text {meta }}\right), 2.85(\mathrm{~s}, 1 \mathrm{H}, \mathrm{C} \equiv \mathrm{C}-H), 2.46\left(\mathrm{~s}, 3 \mathrm{H}, \mathrm{SO}_{2} \mathrm{Ar}-\mathrm{CH}_{3}\right)$, and $0.17\left(\mathrm{~s}, 9 \mathrm{H},-\mathrm{Si}\left(\mathrm{CH}_{3}\right)_{3}\right)$.

${ }^{13}$ C NMR (125 MHz, $\left.\mathrm{CDCl}_{3}\right): \delta$ 145.5, 141.6 (br), 134.5, 131.4 (q, $\left.J=33.3 \mathrm{~Hz}\right), 131.3$ (q, $\left.J=3.7 \mathrm{~Hz}\right), 130.03$, 129.97, 128.7, 125.9 (3.6 Hz), 123.8, 123.4 (q, $J=272.5 \mathrm{~Hz}), 104.2,98.4,74.9,60.1,22.0$, and -0.2.

IR: $3299,2961,2135,1597,1493,1413,1379,1332,1251,1173,1132,884$, and $845 \mathrm{~cm}^{-1}$.

HRMS (ESI-TOF): Calcd for $\mathrm{C}_{21} \mathrm{H}_{20} \mathrm{~F}_{3} \mathrm{NNaO}_{2} \mathrm{SSi}^{+}[\mathrm{M}+\mathrm{Na}]^{+}$requires 458.0828; found 458.0831 .

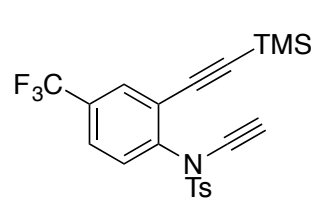

S5

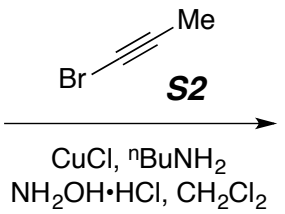

$\mathrm{NH}_{2} \mathrm{OH} \cdot \mathrm{HCl}, \mathrm{CH}_{2} \mathrm{Cl}_{2}$

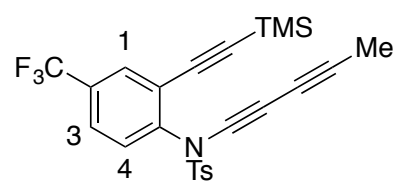

4-(CF3)TMS/Me

Preparation of 4-methyl- $N$-(penta-1,3-diyn-1-yl)- $N$-(4-(trifluoromethyl)-2((trimethylsilyl)ethynyl)phenyl)benzenesulfonamide (4-(CF3)TMS/Me)

Triyne 4-(CF3)TMS/Me was prepared following General Procedure A using the diyne precursor $N$-ethynyl-4methyl- $N$-(2-((trimethylsilyl)ethynyl)phenyl)benzenesulfonamide (S5, $40 \mathrm{mg}, 0.092 \mathrm{mmol})$, 1-bromopropyne (S2, $0.13 \mathrm{~mL}, 1.4 \mathrm{M}$ in hexanes), $\mathrm{CuCl}$ (1 mg, $0.01 \mathrm{mmol}), n-\mathrm{BuNH}_{2}(0.5 \mathrm{~mL})$, and $\mathrm{CH}_{2} \mathrm{Cl}_{2}(0.5 \mathrm{~mL})$.

Purification by flash column chromatography (hexanes: ethyl acetate 12:1 to 5:1) gave the triyne 4-

(CF3)TMS/Me (41.4 mg, $0.087 \mathrm{mmol}, 95 \%)$ as a pale yellow oil.

${ }^{1} \mathbf{H}$ NMR $\left(500 \mathrm{MHz}, \mathrm{CDCl}_{3}\right): \delta 7.72\left(\mathrm{ddq}, J=2.2,0.5,0.8 \mathrm{~Hz}, 1 \mathrm{H}, \mathrm{Ar} H_{l}\right), 7.67(\mathrm{~d}, J=8.3 \mathrm{~Hz}, 2 \mathrm{H}$, $\left.\mathrm{ArSO}_{2} \mathrm{Ar} H_{\text {ortho }}\right), 7.56$ (ddq, $\left.J=8.4,2.2,0.7 \mathrm{~Hz}, 1 \mathrm{H}, \mathrm{Ar} H_{3}\right), 7.45$ (ddq, $\left.J=8.4,0.8,0.4 \mathrm{~Hz}, 1 \mathrm{H}, \mathrm{Ar} H_{4}\right), 7.33(\mathrm{~d}, J$ $\left.=8.1 \mathrm{~Hz}, 2 \mathrm{H}, \mathrm{ArSO}_{2} \mathrm{ArH} H_{\text {meta }}\right), 2.46\left(\mathrm{~s}, 3 \mathrm{H}, \mathrm{SO}_{2} \mathrm{Ar}-\mathrm{CH}_{3}\right), 1.95\left(\mathrm{~s}, 3 \mathrm{H}, \mathrm{C} \equiv \mathrm{C}-\mathrm{CH}_{3}\right)$, and $0.17\left(\mathrm{~s}, 9 \mathrm{H}, \mathrm{Si}\left(\mathrm{CH}_{3}\right)_{3}\right)$.

${ }^{13}$ C NMR (125 MHz, $\left.\mathrm{CDCl}_{3}\right): \delta$ 145.6, 141.5 (br), 134.4, 131.3 (q, $\left.J=33.5 \mathrm{~Hz}\right), 131.3$ (q, $\left.J=3.7 \mathrm{~Hz}\right), 130.1$, 129.9, 128.6, 125.8 (q, $J=3.5 \mathrm{~Hz}), 123.3,123.3(\mathrm{q}, J=273.2 \mathrm{~Hz}), 104.6,98.0,80.9,65.5,63.9,60.1,22.0,4.7$, and -0.3 .

IR: $2960,2920,2258,2173,1492,1414,1382,1330,1251,1174,1133,1122,878$, and $845 \mathrm{~cm}^{-1}$.

HRMS (ESI-TOF): Calcd for $\mathrm{C}_{24} \mathrm{H}_{22} \mathrm{~F}_{3} \mathrm{NNaO}_{2} \mathrm{SSi}^{+}[\mathrm{M}+\mathrm{Na}]^{+}$requires 496.0985; found 496.0987.

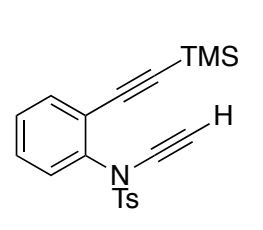

S1

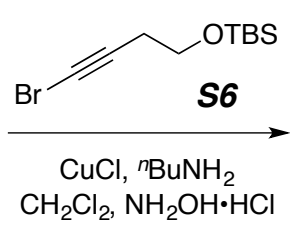

$\mathrm{CH}_{2} \mathrm{Cl}_{2}, \mathrm{NH}_{2} \mathrm{OH} \cdot \mathrm{HCl}$

4-TMS/TOTBS

$N$-(6-((tert-butyldimethylsilyl)oxy)hexa-1,3-diyn-1-yl)-4-methyl- $N$-(2-((trimethylsilyl)ethynyl)phenyl) benzenesulfonamide (4-TMS/tOTBS)

Triyne 4-TMS/tOTBS was prepared following General Procedure A using the diyne precursor $N$-ethynyl-4methyl- $N$-(2-((trimethylsilyl)ethynyl)phenyl)benzenesulfonamide (S1, $20 \mathrm{mg}, 0.054$ mmol), ((4-bromobut-3-yn1-yl)oxy)(tert-butyl)dimethylsilane ${ }^{7}$ (S6, $\left.21.5 \mathrm{mg}, 0.082 \mathrm{mmol}\right), \mathrm{CuCl}$ (1 mg, $\left.0.01 \mathrm{mmol}\right), n-\mathrm{BuNH}_{2}(0.3 \mathrm{~mL})$, 
and $\mathrm{CH}_{2} \mathrm{Cl}_{2}(0.5 \mathrm{~mL})$. Purification by flash column chromatography (hexanes: ethyl acteate 12:1 to 5:1) gave the triyne 4-TMS/tOTBS (17.2 $\mathrm{mg}, 0.031 \mathrm{mmol}, 57 \%)$ as a pale yellow oil.

${ }^{1}$ H NMR $\left(500 \mathrm{MHz}, \mathrm{CDCl}_{3}\right.$ ): $\delta 7.67$ (d, $J=8.3 \mathrm{~Hz}, 2 \mathrm{H}, \mathrm{SO}_{2} \mathrm{ArH}_{\text {ortho }}$ ), 7.47-7.44 (nfom, $\left.1 \mathrm{H}, \mathrm{ArH}\right), 7.33-7.27$ (nfom, 5H, ArH), 3.71 (t, $J=7.3 \mathrm{~Hz}, 2 \mathrm{H}, \mathrm{CH}_{2} \mathrm{OTBS}$ ), 2.51 (t, $J=7.3 \mathrm{~Hz}, 2 \mathrm{H}, \mathrm{CH}_{2} \mathrm{CH}_{2} \mathrm{OTBS}$ ), 2.45 (s, 3H, $\left.\mathrm{SO}_{2} \mathrm{Ar}-\mathrm{CH} H_{3}\right), 0.89$ (s, 9H, $\left.\mathrm{Si}\left(\mathrm{CH}_{3}\right)_{2} \mathrm{C}\left(\mathrm{CH}_{3}\right)_{3}\right), 0.16\left(\mathrm{~s}, 9 \mathrm{H},-\mathrm{Si}\left(\mathrm{CH}_{3}\right)_{3}\right)$, and $0.06\left(\mathrm{~s}, 6 \mathrm{H}, \mathrm{Si}\left(\mathrm{CH}_{3}\right)_{2} \mathrm{C}_{(}\left(\mathrm{CH}_{3}\right)_{3}\right)$.

${ }^{13} \mathbf{C}$ NMR $\left(125 \mathrm{MHz}, \mathrm{CDCl}_{3}\right): \delta 145.1,138.6,134.7,134.1,129.9,129.3$ (2C), 129.1, 128.6, 122.7, 102.1, 99.5, $81.4,67.2,66.2,61.8,59.0,26.1,24.2,22.0,18.5,-0.1$, and -5.1 .

IR: $2955,2928,2857,2253,2169,1380,1250,1175,1104$, and $843 \mathrm{~cm}^{-1}$.

HRMS (ESI-TOF): Calcd for $\mathrm{C}_{30} \mathrm{H}_{39} \mathrm{NNaO}_{3} \mathrm{SSi}_{2}{ }^{+}[\mathrm{M}+\mathrm{Na}]^{+}$requires 572.2081; found 572.2092.

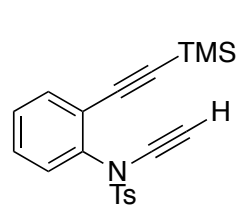

S1

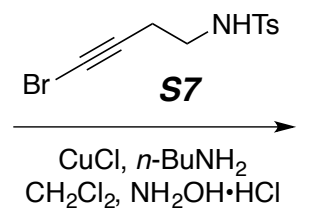

$\mathrm{CH}_{2} \mathrm{Cl}_{2}, \mathrm{NH}_{2} \mathrm{OH} \cdot \mathrm{HC}$

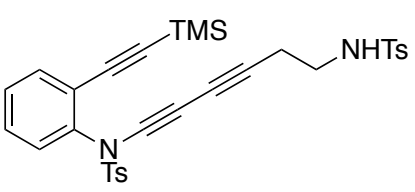

4-TMS/TNHTS

Preparation of 4-methyl- $N$-(6-((4-methylphenyl)sulfonamido)hexa-1,3-diyn-1-yl)- $N$-(2((trimethylsilyl)ethynyl)phenyl)benzenesulfonamide (4-TMS/tNHTs)

Triyne 4-TMS/tNHTs was prepared following General Procedure A using the diyne precursor $N$-ethynyl-4methyl- $N$-(2-((trimethylsilyl)ethynyl)phenyl)benzenesulfonamide (S1, $50 \mathrm{mg}, 0.14 \mathrm{mmol}), N$-(4-bromobut-3yn-1-yl)-4-methylbenzenesulfonamide ${ }^{8}$ (S7, $\left.62 \mathrm{mg}, 0.21 \mathrm{mmol}\right), \mathrm{CuCl}(1 \mathrm{mg}, 0.01 \mathrm{mmol}), n-\mathrm{BuNH}_{2}(1 \mathrm{~mL})$, and $\mathrm{CH}_{2} \mathrm{Cl}_{2}(1 \mathrm{~mL})$. Purification by flash column chromatography (hexanes: ethyl acteate $\left.=3: 1\right)$ gave the triyne 4-TMS/tNHTs (73 mg, $0.12 \mathrm{mmol}, 91 \%$ ) as a pale yellow oil. This yield is of chromatographed material that still contains a small amount of a co-eluting impurity containing $i$-Pr resonances.

${ }^{1}$ H NMR $\left(500 \mathrm{MHz}, \mathrm{CDCl}_{3}\right): \delta 7.74\left(\mathrm{~d}, J=8.2 \mathrm{~Hz}, 2 \mathrm{H}, \mathrm{SO}_{2} \mathrm{Ar}_{a} H_{\text {ortho }}\right), 7.66\left(\mathrm{~d}, J=8.2 \mathrm{~Hz}, 2 \mathrm{H}, \mathrm{SO}_{2} \mathrm{Ar}_{b} H_{\text {ortho }}\right)$, 7.48-7.45 (nfom, 1H, ArH), 7.36-7.28 (nfom, 7H, ArH), 4.77-4.72 (br m, 1H, NHTs), 3.09 (dt, $J=6.5,6.5 \mathrm{~Hz}$, $2 \mathrm{H}, \mathrm{CH}_{2} \mathrm{CH}_{2} \mathrm{NHTs}$ ), 2.46 (t, $J=6.5 \mathrm{~Hz}, 2 \mathrm{H}, \mathrm{CH}_{2} \mathrm{CH}_{2} \mathrm{NHTs}$ ), 2.46 (s, 3H, $\mathrm{SO}_{2} \mathrm{Ar}_{a}-\mathrm{CH}_{3}$ ), 2.42 (s, 3H, $\mathrm{SO}_{2} \mathrm{Ar}_{b^{-}}$ $\left.\mathrm{CH}_{3}\right)$, and $0.16\left(\mathrm{~s}, 9 \mathrm{H}, \mathrm{Si}\left(\mathrm{CH}_{3}\right)_{3}\right)$.

${ }^{13}$ C NMR (125 MHz, $\left.\mathrm{CDCl}_{3}\right): \delta 145.4,143.9,138.3,137.0,134.5,134.2,130.03,130.02,129.4,129.30$, $129.29,128.6,127.3,122.7,102.2,99.5,80.1,68.0,67.4,58.5,41.8,22.0,21.7,21.3$, and -0.1 .

IR: $3292,2962,2252,2169,1597,1482,1445,1378,1331,1161,1090$, and $845 \mathrm{~cm}^{-1}$.

HRMS (ESI-TOF): Calcd for $\mathrm{C}_{31} \mathrm{H}_{32} \mathrm{~N}_{2} \mathrm{NaO}_{4} \mathrm{~S}_{2} \mathrm{Si}^{+}[\mathrm{M}+\mathrm{Na}]^{+}$requires 611.1465; found 611.1494.

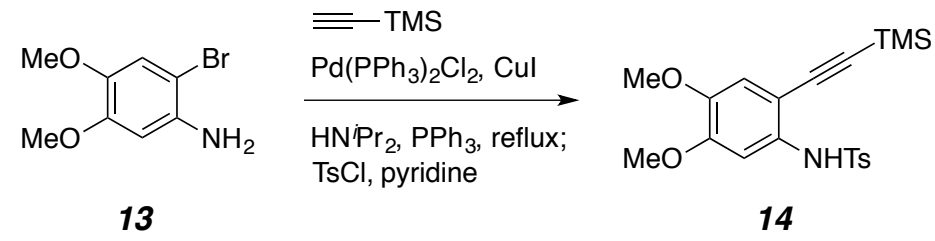

\section{Preparation of $N$-(4,5-dimethoxy-2-((trimethylsilyl)ethynyl)phenyl)-4-methylbenzenesulfonamide (14)}

A mixture of 2-bromo-4,5-dimethoxyaniline ${ }^{9}(\mathbf{1 3}, 7 \mathrm{~g}, 30 \mathrm{mmol})$, ethynyltrimethylsilane (7 $\left.\mathrm{mL}, 49 \mathrm{mmol}\right)$, triphenylphosphine $(510 \mathrm{mg}, 2 \mathrm{mmol})$, and diisopropylamine $(130 \mathrm{~mL})$ were combined in a $200 \mathrm{~mL}$ round bottom flask. A reflux condenser was attached with a rubber septum on the top. Oxygen dissolved in this solution was removed via bubbling of nitrogen gas through an 18-gauge needle with stirring at room 
temperature for $10 \mathrm{~min}$. A powdered mixture of $\mathrm{Pd}\left(\mathrm{PPh}_{3}\right)_{2} \mathrm{Cl}_{2}(680 \mathrm{mg}, 0.97 \mathrm{mmol})$ and $\mathrm{CuI}(185 \mathrm{mg}, 0.97$ mmol) was added. The reaction mixture was kept under a slight positive pressure of $\mathrm{N}_{2}$ and was brought to reflux through heating in an oil bath. After 2 days, the reaction was cooled to room temperature. Additional ethynyltrimethylsilane ( $1 \mathrm{~mL}, 7 \mathrm{mmol})$, triphenylphosphine ( $340 \mathrm{mg}, 1.3 \mathrm{mmol}), \mathrm{Pd}\left(\mathrm{PPh}_{3}\right)_{2} \mathrm{Cl}_{2}(450 \mathrm{mg}, 0.64$ $\mathrm{mmol})$ and $\mathrm{CuI}(120 \mathrm{mg}, 0.63 \mathrm{mmol})$ were added. The solution was sparged again with $\mathrm{N}_{2}$ gas for $10 \mathrm{~min}$. The mixture was heated to reflux temperature for one additional day under a slight positive pressure of $\mathrm{N}_{2}$. This reaction mixture was cooled to room temperature, filtered through a short pad of Celite, and concentrated to give a dark brown oil. This oil was redissolved in pyridine $(60 \mathrm{~mL})$. Toluenesulfonyl chloride $(5.72 \mathrm{~g}, 30 \mathrm{mmol})$ was added and the mixture was allowed to stir at room temperature overnight. This mixture was concentrated, and the residue was partitioned between ethyl acetate and $2 \mathrm{M} \mathrm{HCl} \mathrm{(aq).} \mathrm{The} \mathrm{organic} \mathrm{layer} \mathrm{was} \mathrm{washed} \mathrm{with}$ brine, dried with $\mathrm{Na}_{2} \mathrm{SO}_{4}$, filtered, and concentrated. The residue was purified by flash column chromatrography (hexanes:ethyl acetate $=12: 1$ to 3:1) to give $N$-(4,5-dimethoxy-2-((trimethylsilyl)ethynyl)phenyl)-4methylbenzenesulfonamide (14, $6.8 \mathrm{~g}, 16.8 \mathrm{mmol}, 56 \%)$ as an off-white solid.

${ }^{1} \mathbf{H}$ NMR $\left(500 \mathrm{MHz}, \mathrm{CDCl}_{3}\right): \delta 7.58\left(\mathrm{~d}, J=8.4 \mathrm{~Hz}, 2 \mathrm{H}, \mathrm{SO}_{2} \mathrm{Ar} H_{\text {ortho }}\right), 7.22(\mathrm{~s}, 1 \mathrm{H}, \mathrm{Ar} H), 7.18(\mathrm{~d}, J=8.5 \mathrm{~Hz}$, $\left.2 \mathrm{H}, \mathrm{ArSO}_{2} \mathrm{Ar}_{\text {meta }}\right), 6.93(\mathrm{br} \mathrm{s}, 1 \mathrm{H}, \mathrm{N} H \mathrm{Ts}), 6.72(\mathrm{~s}, 1 \mathrm{H}, \mathrm{Ar} H), 3.92\left[\mathrm{~s}, 3 \mathrm{H}, \mathrm{Ar}\left(\mathrm{OCH}_{3}\right)_{a}\right], 3.79\left[\mathrm{~s}, 3 \mathrm{H}, \operatorname{Ar}(\mathrm{OCH})_{b}\right]$, $2.37\left(\mathrm{~s}, 3 \mathrm{H}, \mathrm{SO}_{2} \mathrm{Ar}-\mathrm{CH} \mathrm{H}_{3}\right)$, and $0.24\left(\mathrm{~s}, 9 \mathrm{H}, \mathrm{C} \equiv \mathrm{C}-\mathrm{Si}\left(\mathrm{CH}_{3}\right)_{3}\right)$.

${ }^{13}$ C NMR (125 MHz, $\left.\mathrm{CDCl}_{3}\right): \delta 150.6,146.3,144.1,136.1,132.7,129.7,127.5,113.6,107.0,105.6,100.4$, $100.0,56.4,56.2,21.8$, and 0.2 .

IR: $3300,2959,2147,1609,1510,1391,1350,1215,1164,1005$, and $846 \mathrm{~cm}^{-1}$.

HRMS (ESI-TOF): Calcd for $\mathrm{C}_{20} \mathrm{H}_{25} \mathrm{NNaO}_{4} \mathrm{SSi}^{+}[\mathrm{M}+\mathrm{Na}]^{+}$requires 426.1166; found 426.1164.

mp: $119-120^{\circ} \mathrm{C}$.
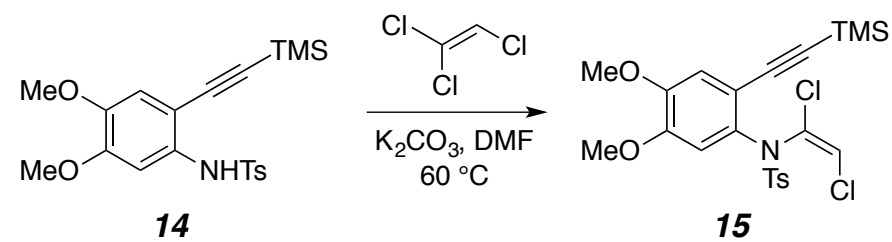

\section{Preparation of $(E)-N$-(1,2-dichlorovinyl)- $N$-(4,5-dimethoxy-2-((trimethylsilyl)ethynyl)phenyl)-4- methylbenzenesulfonamide (15)}

A mixture of $N$-(4,5-dimethoxy-2-((trimethylsilyl)ethynyl)phenyl)-4-methylbenzenesulfonamide (14, 2 g, 5 $\mathrm{mmol}), \mathrm{K}_{2} \mathrm{CO}_{3}(2.1 \mathrm{~g}, 15 \mathrm{mmol})$, trichloroethylene $(1.35 \mathrm{~mL}, 15 \mathrm{mmol})$, and DMF (4 mL) were combined in a $20 \mathrm{~mL}$ vial. This vial was closed with a Teflon-lined cap and placed in a $60{ }^{\circ} \mathrm{C}$ oil bath with stirring overnight. The crude reaction mixture was partitioned between ethyl acetate and brine. The organic layer was dried with anhydrous $\mathrm{Na}_{2} \mathrm{SO}_{4}$, filtered, and concentrated. The residue was purified by column chromatography on silica gel (hexanes:ethyl acetate $=3: 1)$ to give $(E)-N$-(1,2-dichlorovinyl)- $N$-(4,5-dimethoxy-2-

((trimethylsilyl)ethynyl)phenyl)-4-methylbenzenesulfonamide (15, $1.8 \mathrm{~g}, 3.6 \mathrm{mmol})$ as a white foam-like solid.

${ }^{1}$ H NMR $\left(500 \mathrm{MHz}, \mathrm{CDCl}_{3}\right): \delta 7.70\left(\mathrm{~d}, J=8.4 \mathrm{~Hz}, 2 \mathrm{H}, \mathrm{SO}_{2} \mathrm{Ar} H_{\text {ortho }}\right), 7.24\left(\mathrm{~d}, J=8.5 \mathrm{~Hz}, 2 \mathrm{H}, \mathrm{ArSO}_{2} \mathrm{Ar} H_{\text {meta }}\right)$, $7.06(\mathrm{~s}, 1 \mathrm{H}, \mathrm{ArH}), 6.91(\mathrm{~s}, 1 \mathrm{H}, \mathrm{ArH}), 6.36(\mathrm{~s}, 1 \mathrm{H}, \mathrm{NCCl}=\mathrm{CHCl}), 3.88\left[\mathrm{~s}, 3 \mathrm{H}, \mathrm{Ar}\left(\mathrm{OCH}_{3}\right)_{a}\right], 3.80[\mathrm{~s}, 3 \mathrm{H}$, $\left.\mathrm{Ar}\left(\mathrm{OCH}_{3}\right)_{b}\right], 2.42\left(\mathrm{~s}, 3 \mathrm{H}, \mathrm{SO}_{2} \mathrm{Ar}-\mathrm{CH}_{3}\right)$, and $0.15\left(\mathrm{~s}, 9 \mathrm{H}, \mathrm{C} \equiv \mathrm{C}-\mathrm{Si}\left(\mathrm{CH}_{3}\right)_{3}\right)$.

${ }^{13}$ C NMR (125 MHz, $\left.\mathrm{CDCl}_{3}\right): \delta 149.4,149.1,144.7,136.2,131.5,130.8,129.6,129.2,118.5,117.4,116.1$, $115.5,101.6,98.9,56.31,56.26,21.9$, and 0.0 .

IR: 2962, 2159, 1599, 1512, 1353, 1220, 1169, 1073, 1006, and $845 \mathrm{~cm}^{-1}$.

HRMS (ESI-TOF): Calcd for $\mathrm{C}_{22} \mathrm{H}_{25} \mathrm{Cl}_{2} \mathrm{NNaO}_{4} \mathrm{SSi}^{+}[\mathrm{M}+\mathrm{Na}]^{+}$requires 520.0543; found 520.0571. 
mp: $45-47{ }^{\circ} \mathrm{C}$.<smiles>CC#Cc1cc(OC)c(OC)cc1N([12CH3])/C(Cl)=C/Cl</smiles>

15

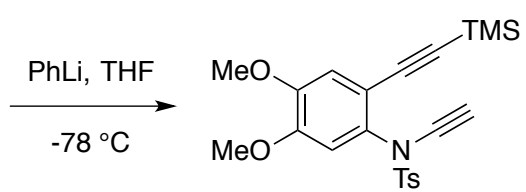

16

Preparation of $\mathrm{N}$-(4,5-dimethoxy-2-((trimethylsilyl)ethynyl)phenyl)- $N$-ethynyl-4-methylbenzenesulfonamide (16)

A solution of (E)-N-(1,2-dichlorovinyl)- $N$-(4,5-dimethoxy-2-((trimethylsilyl)ethynyl)phenyl)-4methylbenzenesulfonamide $(\mathbf{1 5}, 1.5 \mathrm{~g}, 3 \mathrm{mmol})$ in THF $(30 \mathrm{~mL}, 10 \mathrm{~mL} / \mathrm{mmol})$ was cooled to $-78^{\circ} \mathrm{C}$ in a septum-sealed round bottom flask equipped with a $\mathrm{N}_{2}$ line. A solution of PhLi (5.1 mL, 1.3 M in diethyl ether) was added slowly over $10 \mathrm{~min}$ to form an orange-red solution. This mixture was stirred at $-78{ }^{\circ} \mathrm{C}$ for $4 \mathrm{~h}$ before addition of $\mathrm{H}_{2} \mathrm{O} / \mathrm{Et}_{2} \mathrm{O}(2 \mathrm{~mL}, 1: 1)$ at this temperature. The reaction flask was then allowed to warm to room temperature. The reaction mixture was partitioned between ethyl acetate and a saturated aqueous solution of $\mathrm{NH}_{4} \mathrm{Cl}$. The organic layer was washed with brine, dried with anhydrous $\mathrm{Na}_{2} \mathrm{SO}_{4}$, filtered, and concentrated. The residue was purified with column chromatography on silica gel (hexanes:ethyl acetate $=5: 1)$ to give $N-(4,5$ dimethoxy-2-((trimethylsilyl)ethynyl)phenyl)- $N$-ethynyl-4-methylbenzenesulfonamide (16, $1.2 \mathrm{~g}, 2.8 \mathrm{mmol}$, $93 \%)$ as a pale yellow oil.

${ }^{1} \mathbf{H}$ NMR $\left(500 \mathrm{MHz}, \mathrm{CDCl}_{3}\right): \delta 7.71\left(\mathrm{~d}, J=7.9 \mathrm{~Hz}, 2 \mathrm{H}, \mathrm{SO}_{2} \mathrm{Ar} H_{\text {ortho }}\right), 7.30\left(\mathrm{~d}, J=8.0 \mathrm{~Hz}, 2 \mathrm{H}, \mathrm{ArSO}_{2} \mathrm{Ar}_{\text {meta }}\right)$, $6.90(\mathrm{~s}, 1 \mathrm{H}, \mathrm{Ar} H), 6.75(\mathrm{~s}, 1 \mathrm{H}, \mathrm{Ar} H), 3.87\left[\mathrm{~s}, 3 \mathrm{H}, \operatorname{Ar}\left(\mathrm{OCH}_{3}\right)_{a}\right], 3.82\left[\mathrm{~s}, 3 \mathrm{H}, \mathrm{Ar}\left(\mathrm{OCH}_{3}\right)_{b}\right], 2.84(\mathrm{~s}, 1 \mathrm{H}, \mathrm{C} \equiv \mathrm{CH})$, $2.44\left(\mathrm{~s}, 3 \mathrm{H}, \mathrm{SO}_{2} \mathrm{Ar}-\mathrm{CH}_{3}\right)$, and $0.15\left(\mathrm{~s}, 9 \mathrm{H}, \mathrm{C} \equiv \mathrm{C}-\mathrm{Si}\left(\mathrm{CH}_{3}\right)_{3}\right)$.

${ }^{13} \mathbf{C}$ NMR $\left(125 \mathrm{MHz}, \mathrm{CDCl}_{3}\right): \delta 149.7,149.3,145.0,134.7,132.1,129.8,128.7,115.2,115.1,112.3,100.0$, $99.8,75.8,58.9,56.3(2 \mathrm{C}), 21.9$, and 0.1 .

IR: $3294,2966,2160,2133,1600,1511,1374,1223,1173,1003$, and $848 \mathrm{~cm}^{-1}$.

HRMS (ESI-TOF): Calcd for $\mathrm{C}_{22} \mathrm{H}_{25} \mathrm{NNaO}_{4} \mathrm{SSi}^{+}[\mathrm{M}+\mathrm{Na}]^{+}$requires 450.1166; found 450.1172.

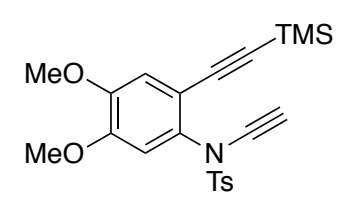

16

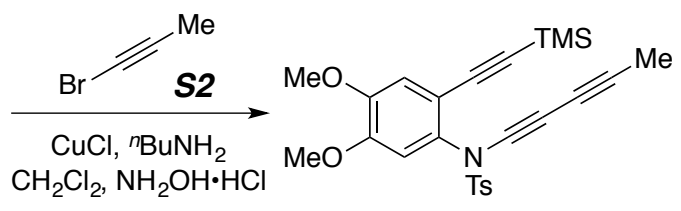

17

\section{Preparation of $N$-(4,5-dimethoxy-2-((trimethylsilyl)ethynyl)phenyl)-4-methyl- $N$-(penta-1,3-diyn-1-yl)- benzenesulfonamide (17)}

Triyne 17 was prepared following General Procedure A using the diyne precursor $N$-(4,5-dimethoxy-2((trimethylsilyl)ethynyl)phenyl)- $N$-ethynyl-4-methylbenzenesulfonamide (16, $630 \mathrm{mg}, 1.47 \mathrm{mmol}), 1$ bromopropyne (S2, $1.7 \mathrm{~mL}, 1.3 \mathrm{M}$ in hexanes), $\mathrm{CuCl}$ (15 mg, $0.15 \mathrm{mmol}), n-\mathrm{BuNH}_{2}(8 \mathrm{~mL})$, and $\mathrm{CH}_{2} \mathrm{Cl}_{2}(8$ $\mathrm{mL}$ ). Purification by flash column chromatography (hexanes:ethyl acetate=5:1) gave the triyne 17 (670 $\mathrm{mg}, 1.44$ mmol, 98\%) as a pale yellow oil.

${ }^{1} \mathbf{H}$ NMR $\left(500 \mathrm{MHz}, \mathrm{CDCl}_{3}\right): \delta 7.68\left(\mathrm{~d}, J=8.3 \mathrm{~Hz}, 2 \mathrm{H}, \mathrm{SO}_{2} \mathrm{ArH}_{\text {ortho }}\right), 7.30\left(\mathrm{~d}, J=8.0 \mathrm{~Hz}, 2 \mathrm{H}, \mathrm{ArSO}_{2} \mathrm{Ar}_{\text {meta }}\right)$, $6.87(\mathrm{~s}, 1 \mathrm{H}, \operatorname{Ar} H), 6.76(\mathrm{~s}, 1 \mathrm{H}, \mathrm{ArH}), 3.87\left[\mathrm{~s}, 3 \mathrm{H}, \operatorname{Ar}\left(\mathrm{OCH}_{3}\right)_{a}\right], 3.83\left[\mathrm{~s}, 3 \mathrm{H}, \operatorname{Ar}\left(\mathrm{OCH}_{3}\right)_{b}\right], 2.45\left(\mathrm{~s}, 3 \mathrm{H}, \mathrm{SO}_{2} \mathrm{Ar}-\right.$ $\left.\mathrm{CH}_{3}\right), 1.95\left(\mathrm{~s}, 3 \mathrm{H}, \mathrm{C} \equiv \mathrm{C}-\mathrm{CH}_{3}\right)$, and $0.15\left(\mathrm{~s}, 9 \mathrm{H}, \mathrm{C} \equiv \mathrm{C}-\mathrm{Si}\left(\mathrm{CH}_{3}\right)_{3}\right)$. 
${ }^{13}$ C NMR (125 MHz, $\left.\mathrm{CDCl}_{3}\right): \delta 149.6,149.2,145.1,134.6,132.1,129.9,128.7,115.1,114.9,112.3,100.2$, $99.7,80.3,66.5,64.2,58.9,56.4,56.3,22.0,4.8$, and -0.1 .

IR: $2965,2258,2161,1601,1510,1375,1220,1173,1033$, and $845 \mathrm{~cm}^{-1}$.

HRMS (ESI-TOF): Calcd for $\mathrm{C}_{25} \mathrm{H}_{27} \mathrm{NNaO}_{4} \mathrm{SSi}^{+}[\mathrm{M}+\mathrm{Na}]^{+}$requires 488.1322; found 488.1338.

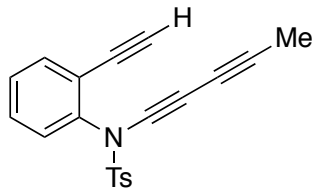

4-H/Me

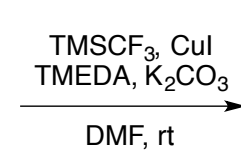

DMF, rt

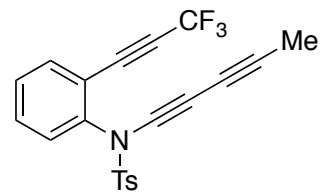

20

Preparation of 4-methyl- $\mathrm{N}$-(penta-1,3-diyn-1-yl)-N-(2-(3,3,3-trifluoroprop-1-yn-1-yl)phenyl)benzenesulfonamide (20) ${ }^{10}$

$\mathrm{CuI}$ (57 mg, $0.3 \mathrm{mmol}, 1.5$ equiv), $\mathrm{K}_{2} \mathrm{CO}_{3}\left(83 \mathrm{mg}, 0.6 \mathrm{mmol}, 3\right.$ equiv), and dry DMF $\left(1.0 \mathrm{~mL}\right.$, ca. $5 \mathrm{~mL} \cdot \mathrm{mmol}^{-1}$ of alkyne substrate) were combined in a $20 \mathrm{~mL}$ scintillation vial equipped with a stir bar and capped with a rubber septum. A large reaction container with a flat bottom is preferred, in order to facilitate $\mathrm{O}_{2}$ dissolution. A balloon filled with dry air was attached. The mixture was stirred vigorously at room temperature while anhydrous TMEDA was introduced via syringe. The white suspension immediately turned blue. This suspension was allowed to stir at ambient temperature for $15 \mathrm{~min}$ before addition of $\mathrm{TMSCF}_{3}(70 \mu \mathrm{L}, 2.4$ eq.) The resulting deep green solution was allowed to stir at room temperature for an additional $5 \mathrm{~min}$ before being cooled to $0{ }^{\circ} \mathrm{C}$ in an ice-water bath. A solution of triyne 4-H/Me (66 mg, $0.20 \mathrm{mmol})$ and $\mathrm{TMSCF}_{3}(70 \mu \mathrm{L}, 2.4 \mathrm{equiv})$ in dry DMF $\left(1.0 \mathrm{~mL}\right.$, ca. $5 \mathrm{~mL} \cdot \mathrm{mmol}^{-1}$ of alkyne substrate) was slowly added over $10 \mathrm{~min}$ at $0{ }^{\circ} \mathrm{C}$. The deep green reaction mixture was allowed to warm to room temperature overnight with stirring. After $16 \mathrm{~h}$ TLC analysis confirmed full consumption of the starting alkyne. The resulting mixture was partitioned between ethyl acetate and brine. The organic layer was washed with brine twice, dried over anhydrous $\mathrm{Na}_{2} \mathrm{SO}_{4}$, and concentrated. The residue was purified by column chromatography on silica gel (hexanes:ethyl acetate $=5: 1$ ) to give the trifluoromethylated triyne $\mathbf{2 0}(73 \mathrm{mg}, 0.18 \mathrm{mmol}, 92 \%)$ as a pale yellow oil.

${ }^{1}$ H NMR (500 MHz, $\left.\mathrm{CDCl}_{3}\right): \delta 7.64\left(\mathrm{~d}, J=8.3 \mathrm{~Hz}, 2 \mathrm{H}, \mathrm{SO}_{2} \mathrm{ArH}{ }_{\text {ortho }}\right), 7.55-7.49(\mathrm{~m}, 2 \mathrm{H}, \mathrm{Ar} H), 7.45(\mathrm{br} \mathrm{d}, J=$ $7.4 \mathrm{~Hz}, 1 \mathrm{H}, \mathrm{Ar} H$ ), 7.39 (br dd, $J=8,8 \mathrm{~Hz}, 1 \mathrm{H}, \mathrm{ArH}$ ), 7.32 (d, $J=8.0 \mathrm{~Hz}, 2 \mathrm{H}, \mathrm{ArSO}_{2} \mathrm{ArH}_{\text {meta }}$ ), 2.45 (s, 3H, $\left.\mathrm{SO}_{2} \mathrm{Ar}-\mathrm{CH}_{3}\right)$, and $1.96\left(\mathrm{~s}, 3 \mathrm{H}, \mathrm{C} \equiv \mathrm{C}-\mathrm{CH}_{3}\right)$.

${ }^{13}$ C NMR (125 MHz, $\left.\mathrm{CDCl}_{3}\right): \delta 146.0,139.7,134.7,133.6,132.0,130.3,130.2,129.5,128.4,118.2,114.5$ (q, J $=257.7), 81.5(\mathrm{q}, J=6.5 \mathrm{~Hz}), 81.0(\mathrm{q}, J=53.2 \mathrm{~Hz}), 80.9,65.8,63.5,59.6,21.9$, and 4.8.

IR: $2258,2174,1487,1448,1381,1316,1175,1143,1090$, and $813 \mathrm{~cm}^{-1}$.

HRMS (ESI-TOF): Calcd for $\mathrm{C}_{21} \mathrm{H}_{14} \mathrm{~F}_{3} \mathrm{NNaO}_{2} \mathrm{~S}^{+}[\mathrm{M}+\mathrm{Na}]^{+}$requires 424.0590; found 424.0607. 
Preparation of carbazole derivatives (i.e., 6, 11, 12, 19, and 22) from HDDA cycloisomerization reaction
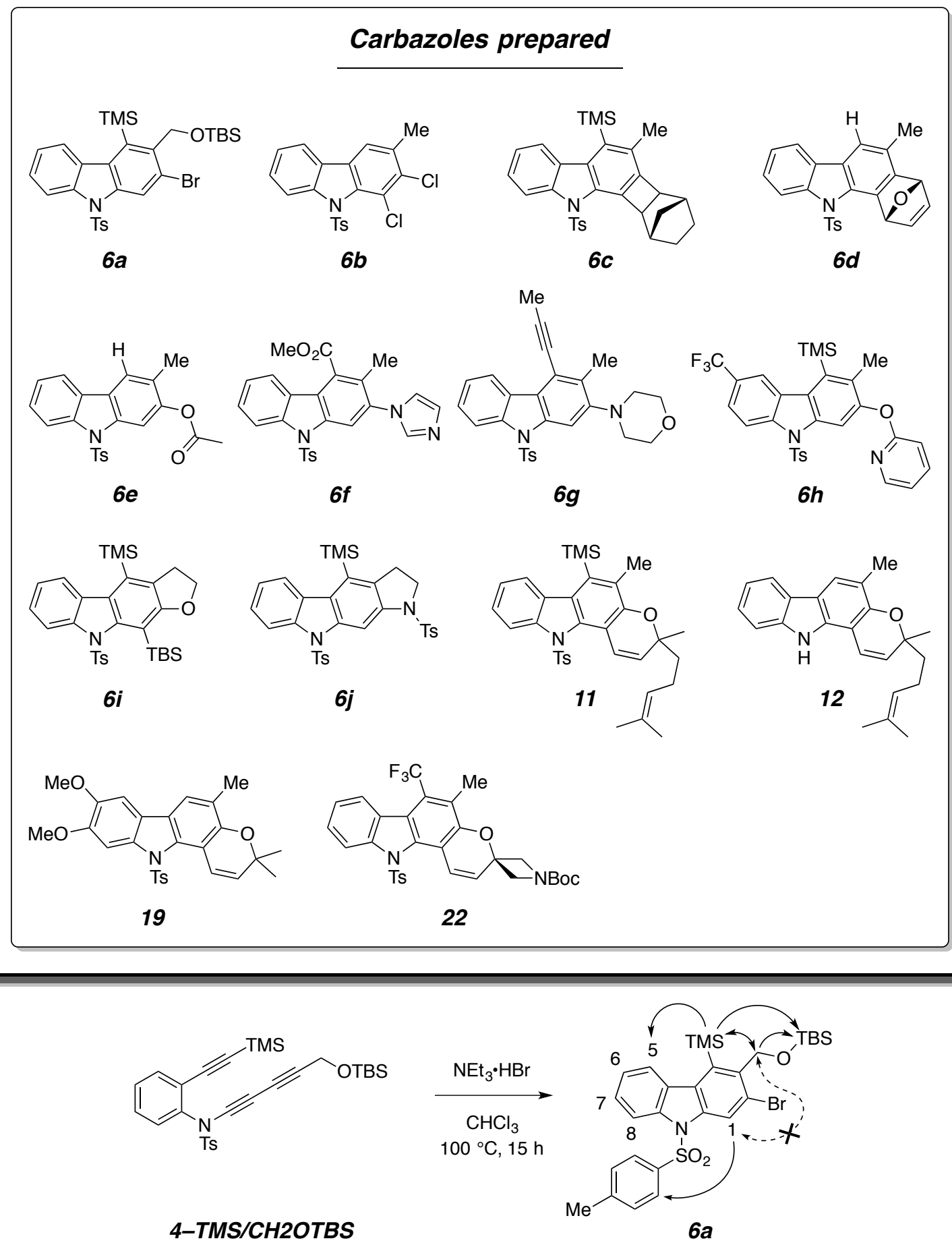

4-TMS/CH2OTBS

$6 a$

\section{Preparation of 2-bromo-3-methyl-9-tosyl-4-(trimethylsilyl)-9H-carbazole (6a)}

Compound 6a was prepared following General Procedure B using 1 ( $25 \mathrm{mg}, 47 \mu \mathrm{mol}), \mathrm{NEt}_{3} \cdot \mathrm{HBr}(18 \mathrm{mg}, 99$ $\mu \mathrm{mol})$, and $\mathrm{CHCl}_{3}(2.3 \mathrm{~mL})$. Purification by column chromatography (hexanes: ethyl acetate $\left.=5: 1\right)$ gave product $6 \mathbf{a}(25 \mathrm{mg}, 41 \mu \mathrm{mol}, 87 \%)$ as a pale yellow oil.

${ }^{1} \mathbf{H}$ NMR $\left(500 \mathrm{MHz}, \mathrm{CDCl}_{3}\right): \delta 8.66\left(\mathrm{~s}, 1 \mathrm{H}, \mathrm{Ar} H_{l}\right), 8.35\left(\mathrm{ddd}, J=8.4,1.1,0.6 \mathrm{~Hz}, 1 \mathrm{H}, \mathrm{Ar} H_{8}\right), 8.06(\mathrm{ddd}, J=$ 8.1, 1.2, $\left.0.6 \mathrm{~Hz}, 1 \mathrm{H}, \mathrm{Ar} H_{5}\right), 7.67$ (d, $J=8.4 \mathrm{~Hz}, 2 \mathrm{H}, \mathrm{ArSO}_{2} \mathrm{Ar}_{\text {ortho }}$ ), 7.46 (ddd, $J=8.4,7.2,1.2 \mathrm{~Hz}, 1 \mathrm{H}, \mathrm{ArH}_{7}$ ), 
7.31 (ddd, $\left.J=8.1,7.3,1.1 \mathrm{~Hz}, 1 \mathrm{H}, \operatorname{Ar} H_{6}\right), 7.12\left(\mathrm{~d}, J=8.7 \mathrm{~Hz}, 2 \mathrm{H}, \mathrm{ArSO}_{2} \mathrm{Ar} H_{\text {meta }}\right), 5.04$ (s, 2H, -CH $\mathrm{CHTBS}_{2} \mathrm{OTB}$ $2.28\left(\mathrm{tt}, J=0.6,0.3 \mathrm{~Hz}, 3 \mathrm{H}, \mathrm{SO}_{2} \mathrm{ArCH}_{3}\right), 0.86\left[\mathrm{~s}, 9 \mathrm{H}, \mathrm{Si}\left(\mathrm{CH}_{3}\right)_{2} \mathrm{C}\left(\mathrm{CH}_{3}\right)_{3}\right], 0.53\left(\mathrm{~s}, 9 \mathrm{H}, \mathrm{Si}\left(\mathrm{CH}_{3}\right)_{3}\right)$, and $0.09[\mathrm{~s}, 6 \mathrm{H}$, $\left.\mathrm{Si}\left(\mathrm{CH}_{3}\right)_{2} \mathrm{C}\left(\mathrm{CH}_{3}\right)_{3}\right]$. Key difference nOe results are indicated by the arrows in structure 6a above. These allow definitive assignment of protons $\mathrm{H} 5 \mathrm{vs}$. $\mathrm{H} 8$ as well as assessment of the sense of regioselectivity in the trapping of the benzyne by the bromide nucleophile.

${ }^{13}$ C NMR (125 MHz, $\left.\mathrm{CDCl}_{3}\right): \delta 145.3,141.4,139.1,138.6,138.4,135.0,131.5,130.0,127.3,126.8(2 \mathrm{C}), 125.7$, $124.2,123.1,120.0,115.1,65.3,26.3,21.7,18.6,3.1$, and -4.5 .

IR: $2954,2928,2856,1598,1567,1459,1368,1251,1187,1174,1082$, and $839 \mathrm{~cm}^{-1}$.

HRMS (ESI-TOF): Calcd for $\mathrm{C}_{29} \mathrm{H}_{38} \mathrm{BrNNaO}_{3} \mathrm{SSi}_{2}{ }^{+}[\mathrm{M}+\mathrm{Na}]^{+}$requires 638.1187; found 638.1188.

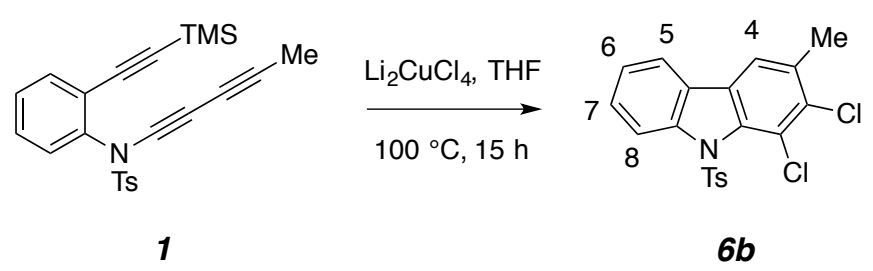

\section{Preparation of 1,2-dichloro-3-methyl-9-tosyl-9H-carbazole (6b)}

Compound $\mathbf{6 b}$ was prepared following General Procedure B using $1(15 \mathrm{mg}, 37 \mu \mathrm{mol}), \mathrm{Li}_{2} \mathrm{CuCl}_{4}(180 \mu \mathrm{L}, 1 \mathrm{M}$ in THF, $180 \mu \mathrm{mol})$, and THF $(2 \mathrm{~mL})$. Purification by column chromatography (hexanes: ethyl acetate $=5: 1)$ gave product $\mathbf{6 b}(14 \mathrm{mg}, 35 \mu \mathrm{mol}, 94 \%)$ as an off-white solid.

${ }^{1} \mathbf{H}$ NMR $\left(500 \mathrm{MHz}, \mathrm{CDCl}_{3}\right): \delta 8.15\left(\mathrm{ddd}, J=8.3,0.8,0.8 \mathrm{~Hz}, 1 \mathrm{H}, \mathrm{Ar} H_{8}\right), 7.68(\mathrm{ddd}, J=7.7,1.3,0.7 \mathrm{~Hz}, 1 \mathrm{H}$, $\left.\operatorname{ArH}_{5}\right), 7.57$ (q, $\left.J=0.8 \mathrm{~Hz}, 1 \mathrm{H}, \operatorname{ArH}_{4}\right), 7.45\left(\mathrm{ddd}, J=8.5,7.3,1.3 \mathrm{~Hz}, 1 \mathrm{H}, \operatorname{Ar} H_{7}\right), 7.40(\mathrm{~d}, J=8.4 \mathrm{~Hz}, 2 \mathrm{H}$, $\mathrm{ArSO}_{2} \mathrm{ArH}_{\text {ortho }}$ ), 7.33 (ddd, $\left.J=7.7,7.3,1.0 \mathrm{~Hz}, 1 \mathrm{H}, \mathrm{Ar} H_{6}\right), 7.05$ (d, $\left.J=8.1 \mathrm{~Hz}, 2 \mathrm{H}, \mathrm{ArSO}_{2} \mathrm{ArH}_{\text {meta }}\right), 2.53(\mathrm{~s}, 3 \mathrm{H}$, $\left.\mathrm{Ar}_{\text {carbazole }} \mathrm{CH}_{3}\right)$, and $2.30\left(\mathrm{~s}, 3 \mathrm{H}, \mathrm{SO}_{2} \mathrm{ArCH}_{3}\right)$.

${ }^{13}$ C NMR (125 MHz, $\left.\mathrm{CDCl}_{3}\right): \delta 144.6,142.3,137.5,135.5,134.8,133.4,130.1,129.3,127.9,127.8,127.1$, $125.6,123.8,119.9,119.4,119.3,22.0$, and 21.8 .

IR: $3205,2923,1738(w), 1452,1422,1349,1167,1095,1033$, and $940 \mathrm{~cm}^{-1}$.

HRMS (ESI-TOF): Calcd for $\mathrm{C}_{20} \mathrm{H}_{15} \mathrm{Cl}_{2} \mathrm{NNaO}_{2} \mathrm{~S}^{+}[\mathrm{M}+\mathrm{Na}]^{+}$requires 426.0093; found 426.0097 .

mp: $212-216^{\circ} \mathrm{C}$.
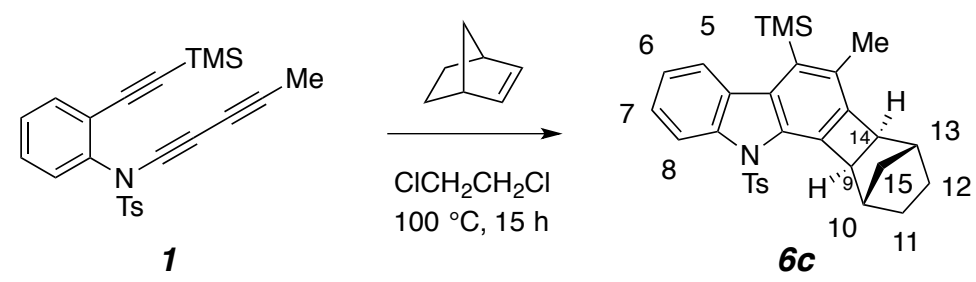

\section{Preparation of 6-methyl-11-tosyl-5-(trimethylsilyl)-6b,8,9,10,10a,11-hexahydro-7H-7,10- methanobenzo[3,4]cyclobuta[1,2-a]carbazole (6c)}

Compound 6c was prepared following General Procedure B using 1 (20 mg, $49 \mu \mathrm{mol}$ ), norbornene (50 mg, 0.53 $\mathrm{mmol})$, dichloroethane $(3 \mathrm{~mL})$. Purification by column chromatography (hexanes: ethyl acetate $=12: 1)$ gave product $\mathbf{6 c}(9 \mathrm{mg}, 18 \mu \mathrm{mol}, 37 \%)$ as an off-white oil.

${ }^{1}$ H NMR $\left(500 \mathrm{MHz}, \mathrm{CDCl}_{3}\right): \delta 8.24\left(\mathrm{~d}, J=8.3 \mathrm{~Hz}, 1 \mathrm{H}, \mathrm{Ar} H_{8}\right), 8.00\left(\mathrm{~d}, J=8.1 \mathrm{~Hz}, 1 \mathrm{H}, \mathrm{Ar} H_{5}\right), 7.60(\mathrm{~d}, J=8.3$ 
$\left.\mathrm{Hz}, 2 \mathrm{H}, \mathrm{ArSO}_{2} \mathrm{ArH}_{\text {ortho }}\right), 7.36\left(\mathrm{dd}, J=8.2,7.4 \mathrm{~Hz}, 1 \mathrm{H}, \mathrm{Ar} H_{7}\right), 7.24\left(\mathrm{dd}, J=8.0,7.3 \mathrm{~Hz}, 1 \mathrm{H}, \mathrm{Ar} H_{6}\right), 7.08(\mathrm{~d}, J=$ $\left.8.4 \mathrm{~Hz}, 2 \mathrm{H}, \mathrm{ArSO}_{2} \mathrm{Ar} H_{\text {meta }}\right), 3.65\left(\mathrm{~d}, J=3.9 \mathrm{~Hz}, 1 \mathrm{H}, \mathrm{H}_{9}\right), 3.20\left(\mathrm{~d}, J=3.7 \mathrm{~Hz}, 1 \mathrm{H}, \mathrm{H}_{14}\right), 2.76$ (br d, $J=2.7 \mathrm{~Hz}$, $\left.1 \mathrm{H}, H_{10}\right), 2.39\left(\mathrm{~s}, 3 \mathrm{H}, \mathrm{Ar}_{\text {carbazole }} \mathrm{CH}_{3}\right), 2.36\left(\mathrm{br} \mathrm{d}, J=2.6 \mathrm{~Hz}, 1 \mathrm{H}, H_{13}\right), 2.27\left(\mathrm{~s}, 3 \mathrm{H}, \mathrm{SO}_{2} \mathrm{ArCH}_{3}\right), 1.72-1.61(\mathrm{~m}$, 2H), 1.37-1.31 (nfom, 1H), 1.30-1.24 (nfom, 1H), 0.98 (d, $J=10.0 \mathrm{~Hz}, 1 \mathrm{H}, \mathrm{C} 15 H_{a} \mathrm{H}_{\mathrm{b}}$ ), 0.86 (br d, $J=10.5 \mathrm{~Hz}$, $\left.1 \mathrm{H}, \mathrm{C} 15 \mathrm{H}_{\mathrm{a}} H_{b}\right)$, and $0.49\left(\mathrm{~s}, 9 \mathrm{H}, \mathrm{Si}\left(\mathrm{CH}_{3}\right)_{3}\right)$.

${ }^{13}$ C NMR (125 MHz, $\left.\mathrm{CDCl}_{3}\right): \delta 146.2,144.5,138.1,135.6,134.3,132.3,131.9,131.6,131.5,129.8,128.4$, $126.8,126.0,125.0,122.5,114.9,52.0,49.6,36.7,36.2,32.1,28.33,28.28,21.7,19.1$, and 3.7 .

IR: 2950, 2869, 1598, 1471, 1421, 1371, 1230, 1175, 1090, and $969 \mathrm{~cm}^{-1}$.

GC-LRMS (ES, $70 \mathrm{eV}): \mathrm{t}_{\mathrm{R}}=16.10 \mathrm{~min} . \mathrm{m} / \mathrm{z}: 499(\mathrm{M})^{\bullet+}, 484(\mathrm{M}-\mathrm{Me})^{+}, 344(\mathrm{M}-\mathrm{Ts})^{+}$.

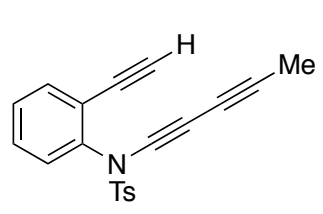

4-H/Me

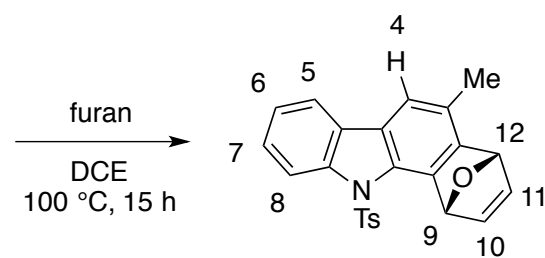

$6 d$

\section{Preparation of 5-methyl-11-tosyl-4,11-dihydro- $1 H$-1,4-epoxybenzo $[a]$ carbazole $(6 \mathrm{~d})$}

Compound 6d was prepared following General Procedure B using 4-H/Me (15 mg, $45 \mu \mathrm{mol})$, furan (15 mg, $0.22 \mathrm{mmol})$, and dichloroethane $(2.5 \mathrm{~mL})$. Purification by column chromatography (hexanes: ethyl acetate $=$ 12:1) gave product $\mathbf{6 d}(13 \mathrm{mg}, 32 \mu \mathrm{mol}, 72 \%)$ as a pale yellow oil.

${ }^{1}$ H NMR $\left(500 \mathrm{MHz}, \mathrm{CDCl}_{3}\right): \delta 8.15\left(\mathrm{dd}, J=8.3,0.8 \mathrm{~Hz}, 1 \mathrm{H}, \mathrm{Ar} H_{8}\right), 7.69\left(\mathrm{ddd}, J=7.7,1.3,0.7 \mathrm{~Hz}, 1 \mathrm{H}, \mathrm{Ar} H_{5}\right)$, $7.39\left(\mathrm{dd}, J=5.6,1.9 \mathrm{~Hz}, 1 \mathrm{H}, H_{10}\right), 7.37\left(\mathrm{ddd}, J=8.4,7.3,1.2 \mathrm{~Hz}, 1 \mathrm{H}, \mathrm{Ar} H_{7}\right), 7.28\left(\mathrm{~s}, 1 \mathrm{H}, H_{4}\right), 7.25(\mathrm{ddd}, J=$ 7.5, 7.5, $\left.1.0 \mathrm{~Hz}, 1 \mathrm{H}, \mathrm{ArH}_{6}\right), 7.24$ (d, $\left.J=8.4 \mathrm{~Hz}, 2 \mathrm{H}, \mathrm{ArSO}_{2} \mathrm{Ar} H_{\text {ortho }}\right), 7.18$ (dd, $\left.J=5.5,1.9 \mathrm{~Hz}, 1 \mathrm{H}, H_{11}\right), 6.97$ (d, $\left.J=8.4 \mathrm{~Hz}, 2 \mathrm{H}, \mathrm{ArSO}_{2} \mathrm{Ar} H_{\text {meta }}\right), 6.57\left(\mathrm{dd}, J=2.0,1.0 \mathrm{~Hz}, 1 \mathrm{H}, H_{9}\right), 5.92\left(\mathrm{dd}, J=2.0,1.0 \mathrm{~Hz}, 1 \mathrm{H}, H_{12}\right), 2.46(\mathrm{~s}$, $\left.3 \mathrm{H}, \mathrm{Ar}_{\text {carbazole }} \mathrm{CH}_{3}\right)$, and $2.21\left(\mathrm{~s}, 3 \mathrm{H}, \mathrm{SO}_{2} \mathrm{ArCH}_{3}\right)$.

${ }^{13}$ C NMR $\left(125 \mathrm{MHz}, \mathrm{CDCl}_{3}\right): \delta 149.5,144.7,143.8,143.3,139.9,137.8,134.1,131.8,129.5,128.8,128.1$, $127.4,126.95,126.91,124.8,120.1,118.0,117.0,84.1,81.3,21.7$, and 18.4.

IR: $2922,1598,1469,1419,1367,1173,1090,1033,964,873$, and $844 \mathrm{~cm}^{-1}$.

HRMS (ESI-TOF): Calcd for $\mathrm{C}_{24} \mathrm{H}_{19} \mathrm{NNaO}_{3} \mathrm{~S}^{+}[\mathrm{M}+\mathrm{Na}]^{+}$requires 424.0978; found 424.0990.

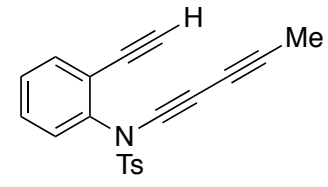

4-H/Me

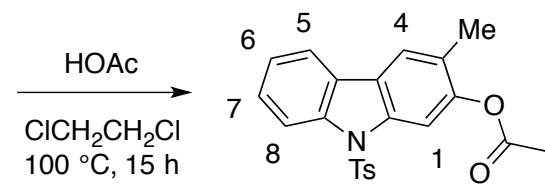

$6 e$

\section{Preparation of 3-methyl-9-tosyl-9H-carbazol-2-yl acetate (6e)}

Compound 6e was prepared following General Procedure B using 4-H/Me (12 mg, $36 \mu \mathrm{mol})$, acetic acid (5 $\mu \mathrm{L}$, $79 \mu \mathrm{mol})$, and dichloroethane $(1.8 \mathrm{~mL})$. Purification by column chromatography (hexanes: ethyl acetate $=5: 1)$ gave product 6e (10 mg, $25 \mu \mathrm{mol}, 71 \%)$ as a pale yellow oil.

${ }^{1}$ H NMR $\left(500 \mathrm{MHz}, \mathrm{CDCl}_{3}\right): \delta 8.28\left(\mathrm{ddd}, J=8.4,1.0,0.8 \mathrm{~Hz}, 1 \mathrm{H}, \mathrm{Ar} H_{8}\right), 8.03\left(\mathrm{dq}, J=0.4,0.4 \mathrm{~Hz}, 1 \mathrm{H}, \mathrm{Ar} H_{l}\right)$, $7.82\left(\mathrm{ddd}, J=7.7,1.3,0.7 \mathrm{~Hz}, 1 \mathrm{H}, \mathrm{Ar} H_{5}\right), 7.71\left(\mathrm{dd}, J=0.8,0.5 \mathrm{~Hz}, 1 \mathrm{H}, \operatorname{Ar} H_{4}\right), 7.68(\mathrm{~d}, J=8.7 \mathrm{~Hz}, 2 \mathrm{H}$, 
$\left.\mathrm{ArSO}_{2} \mathrm{Ar} H_{\text {ortho }}\right), 7.45$ (ddd, $\left.J=8.4,7.3,1.3 \mathrm{~Hz}, 1 \mathrm{H}, \mathrm{Ar} H_{7}\right), 7.33$ (ddd, $\left.J=7.7,7.3,1.0 \mathrm{~Hz}, 1 \mathrm{H}, \operatorname{Ar} H_{6}\right), 7.10(\mathrm{~d}, J$ $\left.=8.7 \mathrm{~Hz}, 2 \mathrm{H}, \mathrm{ArSO}_{2} \mathrm{Ar}_{\text {meta }}\right), 2.41\left(\mathrm{~s}, 3 \mathrm{H}, \mathrm{OC}(\mathrm{O}) \mathrm{CH} H_{3}\right), 2.29\left(\mathrm{dd}, J=0.9,0.4,3 \mathrm{H}, \mathrm{Ar}_{\text {carbazole }} \mathrm{CH}_{3}\right)$, and $2.25(\mathrm{tt}, J$ $\left.=0.7,0.3 \mathrm{~Hz}, 3 \mathrm{H}, \mathrm{SO}_{2} \mathrm{ArCH}_{3}\right)$.

${ }^{13}$ C NMR (125 MHz, $\left.\mathrm{CDCl}_{3}\right): \delta 169.6,148.9,145.2,139.0,137.2,134.8,129.9,127.3,126.8,126.7,126.2$, $124.7,124.2,121.7,120.0,115.4,109.7,21.7,21.1$, and 16.6.

IR: $2970,1739,1448,1366,1227,1216,1205,1169,1033$, and $908 \mathrm{~cm}^{-1}$.

HRMS (ESI-TOF): Calcd for $\mathrm{C}_{22} \mathrm{H}_{19} \mathrm{NNaO}_{4} \mathrm{~S}^{+}[\mathrm{M}+\mathrm{Na}]^{+}$requires 416.0927; found 416.0933.

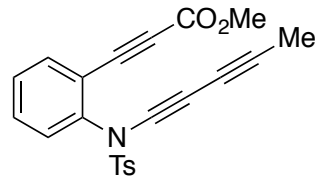

4-E/Me

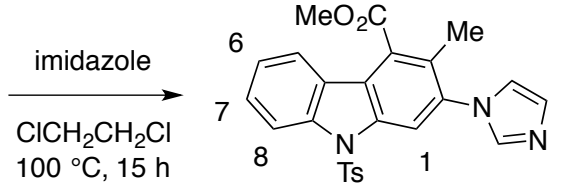

$6 f$

\section{Preparation of methyl 2-(1H-imidazol-1-yl)-3-methyl-9-tosyl-9H-carbazole-4-carboxylate (6f)}

Compound $6 \mathbf{f}$ was prepared following General Procedure B using 4-E/Me (13 mg, $33 \mu \mathrm{mol})$, imidazole (11 mg, $162 \mu \mathrm{mol})$, and dichloroethane $(1.7 \mathrm{~mL})$. Purification by column chromatography (hexanes: ethyl acetate:methanol $=10: 10: 1)$ gave product $\mathbf{6 f}[12 \mathrm{mg}, 26 \mu \mathrm{mol}, 79 \%$, if pure; this material was contaminated with two coeluting impurities to the extent of $7 \mathrm{~mol} \%$ and $2 \mathrm{~mol} \%$ (from ${ }^{1} \mathrm{H}$ NMR integration)] as a brown oil. The sample was rechromatographed and the resulting material showed no appreciable change in the impurity profile (nor its color).

${ }^{1}$ H NMR $\left(400 \mathrm{MHz}, \mathrm{CDCl}_{3}\right): \delta 8.38\left(\mathrm{ddd}, J=8.5,1.0,0.7 \mathrm{~Hz}, 1 \mathrm{H}, \mathrm{Ar} H_{8}\right), 8.33\left(\mathrm{q}, J=0.4 \mathrm{~Hz}, 1 \mathrm{H}, \mathrm{ArH}_{1}\right), 7.70$ (ddd, $\left.J=8.0,1.3,0.7 \mathrm{~Hz}, 1 \mathrm{H}, \mathrm{Ar} H_{5}\right), 7.67$ (d, $J=8.4 \mathrm{~Hz}, 2 \mathrm{H}, \mathrm{ArSO}_{2} \mathrm{ArH}_{\text {ortho }}$ ), 7.66 (dd, $J=1.2,0.9 \mathrm{~Hz}, 1 \mathrm{H}$, $\left.\operatorname{Ar}_{\text {imid }} H_{2}\right), 7.57\left(\mathrm{ddd}, J=8.5,7.3,1.3 \mathrm{~Hz}, 1 \mathrm{H}, \mathrm{Ar} H_{7}\right), 7.38\left(\mathrm{ddd}, J=8.0,7.3,1.0 \mathrm{~Hz}, 1 \mathrm{H}, \operatorname{Ar} H_{6}\right), 7.29(\mathrm{dd}, J=$ $\left.1.3,0.9 \mathrm{~Hz}, 1 \mathrm{H}, \mathrm{Ar}_{\text {imid }} H_{5}\right), 7.16\left(\mathrm{~d}, J=8.6 \mathrm{~Hz}, 2 \mathrm{H}, \mathrm{ArSO}_{2} \mathrm{Ar} H_{\text {meta }}\right), 7.13\left(\mathrm{dd}, J=1.3,1.3 \mathrm{~Hz}, 1 \mathrm{H}, \mathrm{Ar}_{\text {imid }} H_{4}\right), 4.10$ (s, $\left.3 \mathrm{H}, \mathrm{CO}_{2} \mathrm{CH}_{3}\right), 2.31\left(\mathrm{tt}, J=0.6,0.3 \mathrm{~Hz}, 3 \mathrm{H}, \mathrm{SO}_{2} \mathrm{ArCH}_{3}\right.$ ), and 2.17 (d, $J=0.4 \mathrm{~Hz}, 3 \mathrm{H}, \mathrm{Ar}_{\text {carbazole }} \mathrm{CH}_{3}$ ).

${ }^{13}$ C NMR (125 MHz, $\left.\mathrm{CDCl}_{3}\right): \delta 168.9,145.9,139.5,138.0,136.8,135.8,134.7,130.2,130.0,128.9,128.3$, $127.2,126.7,124.6,123.6,123.3,121.6,121.2,115.3,114.9,53.1,21.8$, and 14.8 .

IR: $3111,2954,2927,1731,1597,1494,1465,1372,1215,1174,1091,1072,1048$, and $1028 \mathrm{~cm}^{-1}$.

HRMS (ESI-TOF): Calcd for $\mathrm{C}_{25} \mathrm{H}_{22} \mathrm{~N}_{3} \mathrm{O}_{4} \mathrm{~S}^{+}[\mathrm{M}+\mathrm{H}]^{+}$requires 460.1326; found 460.1332.

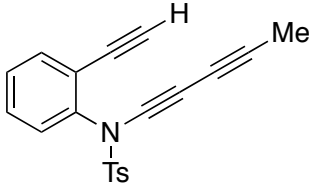

4-H/Me

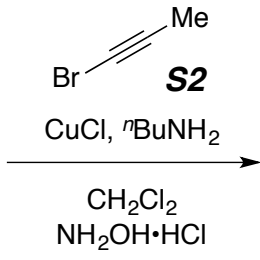

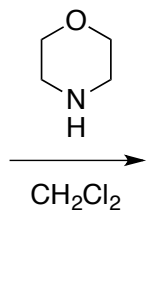

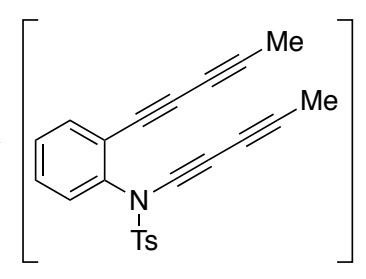

4-propynyl/Me

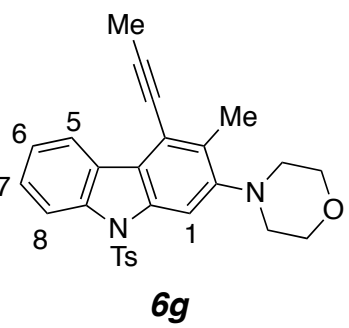

$6 g$

Preparation of 4-(3-methyl-4-(prop-1-yn-1-yl)-9-tosyl-9H-carbazol-2-yl)morpholine (6g)

Tetrayne 4-propynyl/Me was prepared following General Procedure A using triyne precursor $N$-(2ethynylphenyl)-4-methyl- $N$-(penta-1,3-diyn-1-yl)benzenesulfonamide (4-H/Me, $20 \mathrm{mg}, 60 \mu \mathrm{mol}$ ), 1 bromopropyne (0.1 mL, $1.3 \mathrm{M}$ in hexanes), $\mathrm{CuCl}(1 \mathrm{mg}, 0.01 \mathrm{mmol}), n-\mathrm{BuNH}_{2}(0.5 \mathrm{~mL})$, and $\mathrm{CH}_{2} \mathrm{Cl}_{2}(0.5 \mathrm{~mL})$. The total reaction time was ca. $40 \mathrm{~min}$. After aqueous work-up, the crude material was not purified. [A ${ }^{1} \mathrm{H} N \mathrm{NMR}$ 
spectrum of this sample, still containing ethyl acetate, suggested that the tetrayne 4-propynyl/Me had been formed relatively cleanly and showed the following resonances: $\delta 7.71\left(\mathrm{~d}, J=8.4 \mathrm{~Hz}, 2 \mathrm{H}, \mathrm{ArSO}_{2} \mathrm{ArH}_{\text {ortho }}\right.$ ), 7.45 - 7.43 (nfom, 1H), 7.33 (d, $J=8.9 \mathrm{~Hz}, 2 \mathrm{H}, \mathrm{ArSO}_{2} \mathrm{ArH}_{\text {meta }}$ ), 7.35-7.26 (m, 3H), 2.46 (s, 3H, $\mathrm{SO}_{2} \mathrm{ArCH}_{3}$ ), $2.02\left(\mathrm{~s}, 3 \mathrm{H},-\mathrm{C} \equiv \mathrm{CCH}_{3}\right)$, and $1.96\left(\mathrm{~s}, 3 \mathrm{H},-\mathrm{C} \equiv \mathrm{CCH}_{3}\right)$.] The yellow oily residue obtained after concentration was re-dissolved in $\mathrm{CH}_{2} \mathrm{Cl}_{2}$. Morpholine $(30 \mu \mathrm{L}, 0.3 \mathrm{mmol})$ was added. The solution was held at room temperature. After completion of reaction as monitored by TLC (ca. 2 days), the reaction mixture was concentrated. The residue was purified by column chromatography on silica gel (hexanes:ethyl acetate=3:1) to give 4-(3-methyl-4(prop-1-yn-1-yl)-9-tosyl-9H-carbazol-2-yl)morpholine [6g, $22 \mathrm{mg}$, this sample contained 2\% (by mass) of EtOAc (from integration of ${ }^{1} \mathrm{H}$ NMR resonances); the corrected yield is $47 \mu \mathrm{mol}, 78 \%$ ] as a white solid.

${ }^{1}$ H NMR $\left(500 \mathrm{MHz}, \mathrm{CDCl}_{3}\right): \delta 8.52\left(\mathrm{ddd}, J=7.9,1.4,0.6 \mathrm{~Hz}, 1 \mathrm{H}, \mathrm{Ar} H_{5}\right), 8.31(\mathrm{ddd}, J=8.3,1.1,0.7 \mathrm{~Hz}, 1 \mathrm{H}$, $\left.\operatorname{Ar} H_{8}\right), 8.00\left(\mathrm{q}, J=0.4 \mathrm{~Hz}, 1 \mathrm{H}, \mathrm{ArH}_{l}\right), 7.61$ (d, $J=8.6 \mathrm{~Hz}, 2 \mathrm{H}, \mathrm{ArSO}_{2} \mathrm{Ar} H_{\text {ortho }}$ ), 7.44 (ddd, $J=8.3,7.4,1.4 \mathrm{~Hz}$, $\left.1 \mathrm{H}, \mathrm{Ar} H_{7}\right), 7.34$ (ddd, $\left.J=7.7,7.3,1.2 \mathrm{~Hz}, 1 \mathrm{H}, \mathrm{Ar} H_{6}\right), 7.06$ (d, $J=8.1 \mathrm{~Hz}, 2 \mathrm{H}, \mathrm{ArSO}_{2} \mathrm{ArH}_{\text {meta }}$ ), 3.94-3.90 (nfom, $4 \mathrm{H},-\mathrm{CH}_{2} \mathrm{OCH}_{2^{-}}$), 3.03-2.98 (nfom, 4H, $-\mathrm{CH}_{2} \mathrm{NCH}_{2^{-}}$), 2.49 (d, $J=0.3 \mathrm{~Hz}, 3 \mathrm{H}, \mathrm{Ar}_{\text {carbazole }} \mathrm{CH}_{3}$ ), 2.28 (tt, $J=0.7$, $\left.0.3 \mathrm{~Hz}, 3 \mathrm{H}, \mathrm{SO}_{2} \mathrm{ArCH}_{3}\right)$, and $2.24\left(\mathrm{~s}, 3 \mathrm{H},-\mathrm{C} \equiv \mathrm{CCH}_{3}\right)$.

${ }^{13} \mathbf{C}$ NMR $\left(125 \mathrm{MHz}, \mathrm{CDCl}_{3}\right): \delta 151.1,145.0,138.8,137.5,134.9,132.2,129.7,126.81,126.80,126.6,124.0$, $122.2,121.5,118.1,114.9,106.1,96.0,77.5,67.5,52.9,21.7,15.9$, and 5.0.

IR: $2957,2919,2855,2822,1587,1438,1369,1211,1172,1153,1117,1067,1034$, and $961 \mathrm{~cm}^{-1}$.

HRMS (ESI-TOF): Calcd for $\mathrm{C}_{27} \mathrm{H}_{27} \mathrm{~N}_{2} \mathrm{O}_{3} \mathrm{~S}^{+}[\mathrm{M}+\mathrm{H}]^{+}$requires 459.1737; found 459.1748.

mp: $217-218^{\circ} \mathrm{C}$.

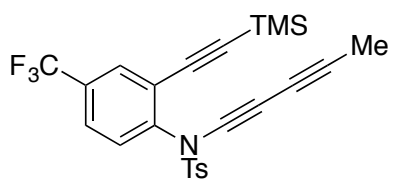

4-(CF3)TMS/Me

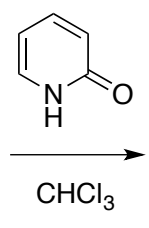

$6 h$

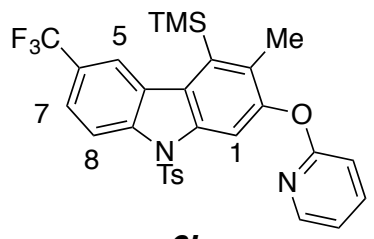

\section{Preparation of 3-methyl-2-(pyridin-2-yloxy)-9-tosyl-4-(trimethylsilyl)-9H-carbazole (6h)}

Compound 6h was prepared following General Procedure B using 4-(CF3)TMS/Me (19 mg, $0.04 \mathrm{mmol})$, 2pyridone $\left(38 \mathrm{mg}, 0.40 \mathrm{mmol}\right.$ ), and $\mathrm{CHCl}_{3}(4 \mathrm{~mL})$. Purification by column chromatography (hexanes: ethyl acetate $=5: 1)$ gave product $\mathbf{6 h}(22.2 \mathrm{mg}, 0.039 \mathrm{mmol}, 97 \%)$ as an off-white solid.

${ }^{1}$ H NMR $\left(500 \mathrm{MHz}, \mathrm{CDCl}_{3}\right): \delta 8.47$ (ddq, $\left.J=8.8,0.7,0.7 \mathrm{~Hz}, 1 \mathrm{H}, \mathrm{Ar} H_{8}\right), 8.33(\mathrm{ddq}, J=1.9,0.7,0.7 \mathrm{~Hz}, 1 \mathrm{H}$, $\left.\operatorname{Ar} H_{5}\right), 8.20\left(\mathrm{ddd}, J=5.0,2.0,0.8 \mathrm{~Hz}, 1 \mathrm{H}, \mathrm{Ar}_{\text {pyridine }} H_{6}\right), 8.17$ (q, $\left.J=0.5 \mathrm{~Hz}, 1 \mathrm{H}, \operatorname{Ar} H_{1}\right), 7.75(\mathrm{ddd}, J=8.3,7.2$, $\left.2.0 \mathrm{~Hz}, 1 \mathrm{H}, \mathrm{Ar}_{\text {pyridine }} H_{4}\right), 7.69$ (d, $J=8.5 \mathrm{~Hz}, 2 \mathrm{H}, \mathrm{ArSO}_{2} \mathrm{Ar}_{\text {ortho }}$ ), 7.67 (ddq, $\left.J=8.8,1.8,0.5 \mathrm{~Hz}, 1 \mathrm{H}, \mathrm{ArH}_{7}\right), 7.14$ $\left(\mathrm{d}, J=8.1 \mathrm{~Hz}, 2 \mathrm{H}, \mathrm{ArSO}_{2} \mathrm{Ar} H_{\text {meta }}\right), 7.04$ (ddd $\left.J=7.2,5.0,0.9 \mathrm{~Hz}, 1 \mathrm{H}, \mathrm{Ar}_{\text {pyridine }} H_{5}\right), 6.99(\mathrm{dt}, J=8.3,0.9 \mathrm{~Hz}, 1 \mathrm{H}$, $\left.\mathrm{Ar}_{\text {pyridine }} H_{3}\right), 2.41\left(\mathrm{~d}, J=0.5 \mathrm{~Hz}, 3 \mathrm{H}, \mathrm{Ar}_{\text {carbazole }} \mathrm{CH}_{3}\right), 2.30\left(\mathrm{~s}, 3 \mathrm{H}, \mathrm{SO}_{2} \mathrm{ArCH}_{3}\right)$, and $0.53\left(\mathrm{~s}, 9 \mathrm{H}, \mathrm{Si}\left(\mathrm{CH}_{3}\right)_{3}\right)$.

${ }^{13}$ C NMR (125 MHz, $\left.\mathrm{CDCl}_{3}\right): \delta 163.9,152.1,147.9,145.6,140.8,139.9,138.1,136.4,134.8,134.1,130.1$, 127.6, 126.9, 125.1, 125.0 (q, $J=32.3 \mathrm{~Hz}), 124.8$ (q, $J=272.2 \mathrm{~Hz}), 123.0$ (q, $J=3.5 \mathrm{~Hz}), 122.3(\mathrm{q}, J=4.4 \mathrm{~Hz})$, $118.7,114.9,111.5,109.8,21.8,18.3$, and 3.3. These carbon NMR chemical shifts compare much more favorably with literature reports of 2-aryloxypyridine derivatives than those of $N$-aryl-2-pyridone derivatives. ${ }^{11}$

IR: $2927,1595,1572,1468,1430,1372,1326,1269,1169,1120,1050$, and $846 \mathrm{~cm}^{-1}$.

HRMS (ESI-TOF): Calcd for $\mathrm{C}_{29} \mathrm{H}_{27} \mathrm{~F}_{3} \mathrm{~N}_{2} \mathrm{NaO}_{3} \mathrm{SSi}^{+}[\mathrm{M}+\mathrm{Na}]^{+}$requires 591.1356; found 591.1368.

mp: $149-151^{\circ} \mathrm{C}$. 


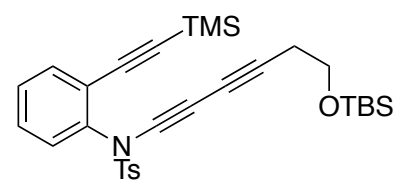

4-TMS/TOTBS
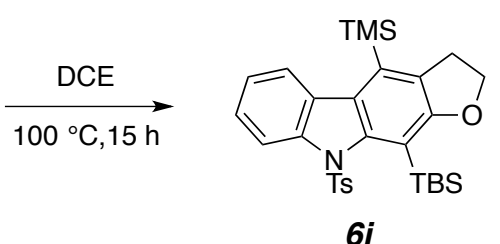

Preparation of 10-(tert-butyldimethylsilyl)-9-tosyl-4-(trimethylsilyl)-3,9-dihydro-2H-furo[2,3-b]carbazole (6i)

In a glass vial was added triyne precursor 4-TMS/tOTBS (12 mg, $0.022 \mathrm{mmol})$ and DCE (1 mL). The vial was sealed with a Teflon-lined cap and placed in a $100{ }^{\circ} \mathrm{C}$ oil bath for $15 \mathrm{~h}$. The crude reaction mixture was concentrated and directly subjected to column chromatographic purification on silica gel (hexanes:ethyl acetate $=5: 1)$ to give $6 \mathbf{i}(10 \mathrm{mg}, 0.018 \mathrm{mmol}, 83 \%)$ as a pale yellow oil.

${ }^{1}$ H NMR $\left(500 \mathrm{MHz}, \mathrm{CDCl}_{3}\right): \delta 8.06\left(\mathrm{ddd}, J=8.0,1.2,0.6 \mathrm{~Hz}, 1 \mathrm{H}, \mathrm{Ar} H_{8}\right), 7.47(\mathrm{ddd}, J=7.9,1.2,0.6 \mathrm{~Hz}, 1 \mathrm{H}$, $\left.\operatorname{Ar} H_{5}\right), 7.23\left(\mathrm{ddd}, J=8.1,7.3,1.2 \mathrm{~Hz}, 1 \mathrm{H}, \mathrm{Ar} H_{7}\right), 7.14\left(\mathrm{ddd}, J=7.9,7.3,1.2 \mathrm{~Hz}, 1 \mathrm{H}, \mathrm{ArH}_{6}\right), 6.70(\mathrm{~d}, J=8.2 \mathrm{~Hz}$, $\left.2 \mathrm{H}, \mathrm{ArSO}_{2} \mathrm{ArH}_{\text {ortho }}\right), 6.67$ (d, $J=8.5 \mathrm{~Hz}, 2 \mathrm{H}, \mathrm{ArSO}_{2} \mathrm{ArH}_{\text {meta }}$ ), 4.69 (ddd, $J=10.0,8.5,5.0 \mathrm{~Hz}, 1 \mathrm{H}, \mathrm{CH}_{a} \mathrm{H}_{\mathrm{b}} \mathrm{O}$ ), 4.46 (ddd, $\left.J=9.6,9.6,8.5 \mathrm{~Hz}, 1 \mathrm{H}, \mathrm{CH}_{\mathrm{a}} H_{b} \mathrm{O}\right), 3.44$ (ddd, $\left.J=15.1,10.1,9.5 \mathrm{~Hz}, 1 \mathrm{H}, \mathrm{CH}_{a} \mathrm{H}_{\mathrm{b}} \mathrm{CH}_{2} \mathrm{O}\right), 3.25$ (ddd, $J$ $\left.=15.1,9.6,5.1 \mathrm{~Hz}, 1 \mathrm{H}, \mathrm{CH}_{\mathrm{a}} \mathrm{H}_{b} \mathrm{CH}_{2} \mathrm{O}\right), 2.14\left(\mathrm{~s}, 3 \mathrm{H}, \mathrm{SO}_{2} \mathrm{Ar}-\mathrm{CH}_{3}\right), 1.06\left(\mathrm{~s}, 9 \mathrm{H}, \mathrm{Si}\left(\mathrm{CH}_{3}\right)_{2} \mathrm{C}\left(\mathrm{CH}_{3}\right)_{3}\right), 0.68(\mathrm{~s}, 3 \mathrm{H}$, $\left.\mathrm{Si}\left(\mathrm{CH}_{3}\right)_{\mathrm{a}}\left(\mathrm{CH}_{3}\right)_{\mathrm{b}} \mathrm{C}\left(\mathrm{CH}_{3}\right)_{3}\right), 0.33\left(\mathrm{~s}, 3 \mathrm{H}, \mathrm{Si}\left(\mathrm{CH}_{3}\right)_{\mathrm{a}}\left(\mathrm{CH}_{3}\right)_{\mathrm{b}} \mathrm{C}\left(\mathrm{CH}_{3}\right)_{3}\right)$, and $0.28\left(\mathrm{~s}, 9 \mathrm{H},-\mathrm{Si}\left(\mathrm{CH}_{3}\right)_{3}\right)$.

${ }^{13}$ C NMR $\left(125 \mathrm{MHz}, \mathrm{CDCl}_{3}\right): \delta 164.6,147.6,143.8,142.1,132.3,130.7,130.6,130.3,129.5,128.0,127.6$, $125.0,124.7,122.8,120.4,115.1,70.7,32.3,29.3,21.5,18.1,1.8,-0.1$, and -0.6 .

IR: 2950, 2929, 2894, 2855, 1461, 1364, 1315, 1254, 1171, 1090, 1039, and $839 \mathrm{~cm}^{-1}$.

HRMS (ESI-TOF): Calcd for $\mathrm{C}_{30} \mathrm{H}_{39} \mathrm{NNaO}_{3} \mathrm{SSi}_{2}{ }^{+}[\mathrm{M}+\mathrm{Na}]^{+}$requires 572.2081; found 572.2067.

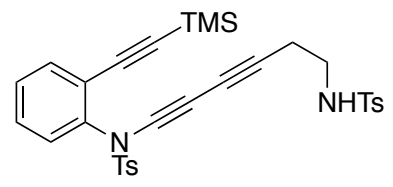

4-TMS/tNHTS

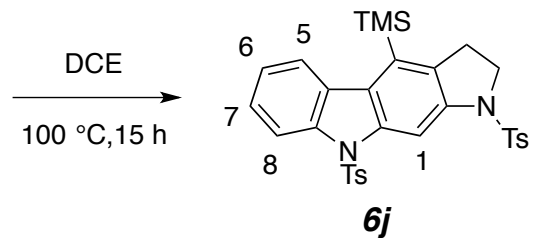

$6 j$

\section{Preparation of 1,9-ditosyl-4-(trimethylsilyl)-1,2,3,9-tetrahydropyrrolo[2,3-b]carbazole (6j)}

In a glass vial was added triyne precursor 4-TMS/tNHTs $(25 \mathrm{mg}, 0.042 \mathrm{mmol})$ and DCE $(2 \mathrm{~mL})$. The vial was sealed with a Teflon-lined cap and placed in a $100{ }^{\circ} \mathrm{C}$ oil bath for $15 \mathrm{~h}$. The crude reaction mixture was concentrated and directly subjected to column chromatography (hexanes:ethyl acetate $=3: 1)$ to give $\mathbf{6 j}(18 \mathrm{mg}$, $0.018 \mathrm{mmol}, 72 \%$ ) as a pale yellow oil.

${ }^{1}$ H NMR $\left(500 \mathrm{MHz}, \mathrm{CDCl}_{3}\right): \delta 8.63\left(\mathrm{~s}, 1 \mathrm{H}, \mathrm{Ar} H_{1}\right), 8.44\left(\mathrm{~d}, J=8.4 \mathrm{~Hz}, 1 \mathrm{H}, \mathrm{Ar} H_{8}\right), 7.93(\mathrm{~d}, J=8.0 \mathrm{~Hz}, 1 \mathrm{H}$, $\left.\operatorname{Ar} H_{5}\right), 7.802\left(\mathrm{~d}, J=8.4 \mathrm{~Hz}, 2 \mathrm{H}, \mathrm{SO}_{2} \mathrm{Ar}_{a} H_{\text {ortho }}\right), 7.796\left(\mathrm{~d}, J=8.4 \mathrm{~Hz}, 2 \mathrm{H}, \mathrm{SO}_{2} \mathrm{Ar}_{b} H_{\text {ortho }}\right.$ ), 7.41 (ddd, $J=8.5,7.2$, $\left.1.2 \mathrm{~Hz}, 1 \mathrm{H}, \mathrm{Ar} H_{7}\right), 7.28\left(\mathrm{ddd}, J=8.1,7.3,1.1 \mathrm{~Hz}, 1 \mathrm{H}, \mathrm{Ar} H_{6}\right), 7.24\left(\mathrm{~d}, J=8.1 \mathrm{~Hz}, 2 \mathrm{H}, \mathrm{SO}_{2} \mathrm{Ar}_{a} H_{m e t a}\right), 7.15(\mathrm{~d}, J=$ $8.1 \mathrm{~Hz}, 2 \mathrm{H}, \mathrm{SO}_{2} \mathrm{Ar}_{b} H_{\text {meta }}$ ), 3.97 (t, $J=8.3 \mathrm{~Hz}, 2 \mathrm{H}, \mathrm{CH}_{2} \mathrm{CH}_{2} \mathrm{NTs}$ ), 3.11 (t, $J=8.3 \mathrm{~Hz}, 2 \mathrm{H}, \mathrm{CH}_{2} \mathrm{CH}_{2} \mathrm{NTs}_{\text {) }}, 2.36$ (s, $\left.3 \mathrm{H}, \mathrm{SO}_{2} \mathrm{Ar}_{a}-\mathrm{CH}_{3}\right), 2.30\left(\mathrm{~s}, 3 \mathrm{H}, \mathrm{SO}_{2} \mathrm{Ar}_{b}-\mathrm{CH}_{3}\right)$, and $0.43\left(\mathrm{~s}, 9 \mathrm{H}, \mathrm{Si}\left(\mathrm{CH}_{3}\right)_{3}\right)$.

${ }^{13}$ C NMR (125 MHz, $\left.\mathrm{CDCl}_{3}\right): \delta 145.0,144.3,140.7,139.2,138.3,135.4,134.0,133.9,130.5,130.0,129.9$, $127.9,127.1,126.4,126.2,126.0,123.9,122.8,114.8,102.0,50.4,30.3,21.8,21.7$, and 2.4.

IR: $2949,1599,1578,1465,1401,1356,1265,1166,1091,1052,1033$, and $844 \mathrm{~cm}^{-1}$.

HRMS (ESI-TOF): Calcd for $\mathrm{C}_{31} \mathrm{H}_{32} \mathrm{~N}_{2} \mathrm{NaO}_{4} \mathrm{~S}_{2} \mathrm{Si}^{+}[\mathrm{M}+\mathrm{Na}]^{+}$requires 611.1465; found 611.1470. 


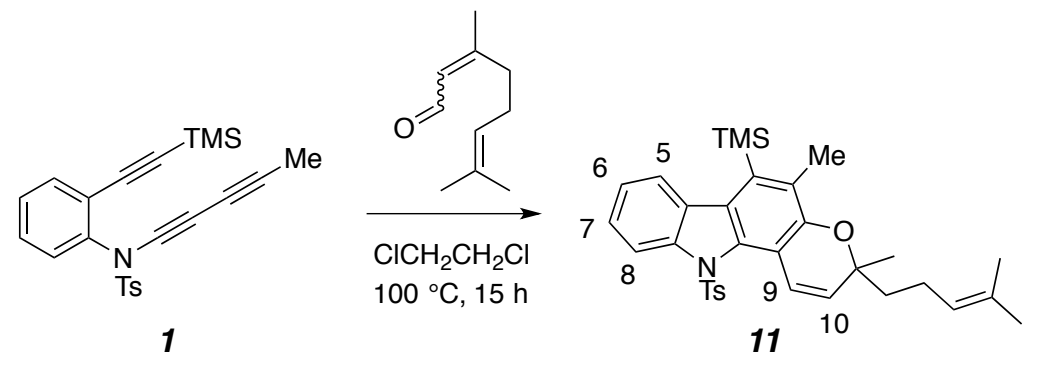

Preparation of 3,5-dimethyl-3-(4-methylpent-3-en-1-yl)-11-tosyl-6-(trimethylsilyl)-3,11dihydropyrano[3,2-a] carbazole (11)

Compound 11 was prepared following General Procedure B using 1 (40 mg, $99 \mu \mathrm{mol}$ ), citral (30 mg, 0.20 $\mathrm{mmol}$ ), and DCE $(5 \mathrm{~mL})$. Purification by column chromatography (hexanes:ethyl acetate $=5: 1$ ) gave product 11 (49 $\mathrm{mg}, 88 \mu \mathrm{mol}, 89 \%$ ) as a pale yellow oil.

${ }^{1} \mathbf{H}$ NMR $\left(500 \mathrm{MHz}, \mathrm{CDCl}_{3}\right): \delta 8.11\left(\mathrm{ddd}, J=8.0,1.2,0.6 \mathrm{~Hz}, 1 \mathrm{H}, \mathrm{Ar} H_{8}\right), 7.50(\mathrm{ddd}, J=7.9,1.2,0.6 \mathrm{~Hz}, 1 \mathrm{H}$, $\left.\mathrm{Ar}_{5}\right), 7.25\left(\mathrm{ddd}, J=8.1,7.4,1.2 \mathrm{~Hz}, 1 \mathrm{H}, \mathrm{Ar} H_{7}\right), 7.23\left(\mathrm{~d}, J=9.8 \mathrm{~Hz}, 1 \mathrm{H}, \mathrm{Ar} H_{9}\right), 7.18$ (ddd, $J=7.9,7.3,1.2 \mathrm{~Hz}$, $\left.\operatorname{Ar} H_{6}\right), 6.87\left(\mathrm{~d}, J=8.3 \mathrm{~Hz}, 2 \mathrm{H}, \mathrm{SO}_{2} \mathrm{Ar} H_{\text {ortho }}\right), 6.77\left(\mathrm{~d}, J=8.1 \mathrm{~Hz}, 2 \mathrm{H}, \mathrm{SO}_{2} \mathrm{Ar} H_{\text {meta }}\right), 5.71\left(\mathrm{~d}, J=9.9 \mathrm{~Hz}, 1 \mathrm{H}, H_{10}\right)$, 5.16 [tqq, $J=7.2,1.4,1.4 \mathrm{~Hz}, 1 \mathrm{H}, \mathrm{CH}_{2} \mathrm{CH}=\mathrm{C}\left(\mathrm{CH}_{3}\right)_{2}$ ], 2.35 (s, 3H, $\left.\mathrm{Ar}_{\text {carbazole }} \mathrm{CH}_{3}\right), 2.19$ (dt, $J=8.3,7.5 \mathrm{~Hz}, 2 \mathrm{H}$, $\left.\mathrm{CH}_{2} \mathrm{CH}=\mathrm{C}\left(\mathrm{CH}_{3}\right)_{2}\right), 2.16\left(\mathrm{~s}, 3 \mathrm{H}, \mathrm{SO}_{2} \mathrm{ArCH}_{3}\right), 1.86-1.79\left(\mathrm{~m}, 2 \mathrm{H}, \mathrm{CH}_{2} \mathrm{CH}_{2} \mathrm{CH}=\right), 1.68(\mathrm{~d}, J=1.1 \mathrm{~Hz}, 3 \mathrm{H}$, $\mathrm{CH}=\mathrm{C}\left(\mathrm{CH}_{3}\right)_{a}\left(\mathrm{CH}_{3}\right)_{b}, 1.59\left(\mathrm{~d}, J=0.8 \mathrm{~Hz}, 3 \mathrm{H}, \mathrm{CH}=\mathrm{C}\left(\mathrm{CH}_{3}\right)_{a}\left(\mathrm{CH}_{3}\right)_{b}\right), 1.52\left(\mathrm{~s}, 3 \mathrm{H}, \mathrm{C}\left(\mathrm{CH}_{3}\right) \mathrm{CH}_{2} \mathrm{CH}_{2}\right)$, and $0.26(\mathrm{~s}$, 9H, $\left.\mathrm{Si}\left(\mathrm{CH}_{3}\right)_{3}\right)$.

${ }^{13}$ C NMR $\left(125 \mathrm{MHz}, \mathrm{CDCl}_{3}\right): \delta 150.5,144.2,141.9,136.6,133.9,132.5,131.8,131.0,130.8,129.7,128.3$, $127.5,127.5,125.1,125.0,124.4,123.6,122.3,120.3,115.1,78.4,41.1,26.2,25.9,23.0,21.5,17.9,17.8$, and 2.9 .

IR: $2969,2924,1598,1538,1453,1367,1306,1253,1174,1075$, and $841 \mathrm{~cm}^{-1}$.

HRMS (ESI-TOF): Calcd for $\mathrm{C}_{33} \mathrm{H}_{39} \mathrm{NNaO}_{3} \mathrm{SSi}^{+}[\mathrm{M}+\mathrm{Na}]^{+}$requires 580.2312; found 580.2330.

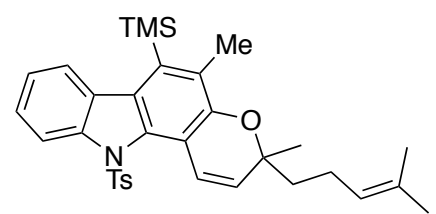

11

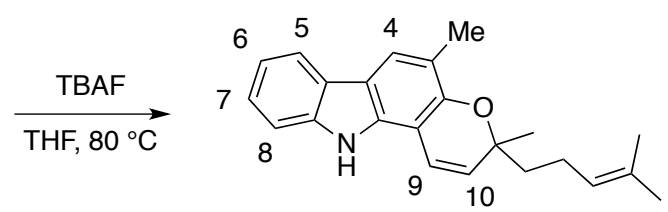

12

\section{Preparation of mahanimbine (12)}

In a glass vial was added 11 (15 mg, $0.027 \mathrm{mmol})$, THF $(0.3 \mathrm{~mL})$, and TBAF $(0.2 \mathrm{~mL}, 1 \mathrm{M}$ in THF) at room temperature. The vial was sealed with a Teflon-lined cap and placed in an $80{ }^{\circ} \mathrm{C}$ oil bath for $6 \mathrm{~h}$. The reaction mixture was partitioned between $\mathrm{NH}_{4} \mathrm{Cl}$ (aq) and ethyl acetate. The organic layer was washed with brine, dried over anhydrous $\mathrm{Na}_{2} \mathrm{SO}_{4}$, filtered, and concentrated. The residue was purified by column chromatography (hexanes: ethyl acetate $=5: 1)$ to give mahanimbine $(\mathbf{1 2}, 8.1 \mathrm{mg}, 0.024 \mathrm{mmol}, 91 \%)$ as a pale yellow oil. The NMR data were essentially identical with those previously reported. ${ }^{12}$

${ }^{1} \mathbf{H}$ NMR $\left(500 \mathrm{MHz}, \mathrm{CDCl}_{3}\right): \delta 7.91$ (ddd, $\left.J=7.8,1.2,0.6 \mathrm{~Hz}, 1 \mathrm{H}, \mathrm{Ar} H_{5}\right), 7.86$ (br s, $\left.1 \mathrm{H}, \mathrm{NH}\right), 7.66(\mathrm{~s}, 1 \mathrm{H}$, $\left.\mathrm{ArH}_{4}\right), 7.37$ (ddd, $\left.J=8.0,1.1,0.6 \mathrm{~Hz}, 1 \mathrm{H}, \mathrm{Ar} H_{8}\right), 7.30\left(\mathrm{ddd}, J=8.0,7.2,1.2 \mathrm{~Hz}, 1 \mathrm{H}, \mathrm{Ar} H_{7}\right), 7.17(\mathrm{ddd}, J=7.7$, $\left.7.2,1.1 \mathrm{~Hz}, 1 \mathrm{H}, \operatorname{Ar} H_{6}\right), 6.64\left(\mathrm{~d}, J=9.8 \mathrm{~Hz}, 1 \mathrm{H}, H_{9}\right), 5.66\left(\mathrm{~d}, J=9.8 \mathrm{~Hz}, 1 \mathrm{H}, H_{10}\right), 5.11$ (tqq, $J=7.2,1.4,1.4 \mathrm{~Hz}$, $\left.1 \mathrm{H}, \mathrm{CH}_{2} \mathrm{CH}=\mathrm{C}\left(\mathrm{CH}_{3}\right)_{2}\right), 2.33$ (s, 3H, $\left.\mathrm{Ar}_{\text {carbazole }} \mathrm{CH}_{3}\right), 2.24-2.10\left(\mathrm{~m}, 2 \mathrm{H}, \mathrm{CH}_{2} \mathrm{CH}=\mathrm{C}\left(\mathrm{CH}_{3}\right)_{2}\right), 1.79-1.74$ (nfom, 2H, $\left.\mathrm{CH}_{2} \mathrm{CH}_{2} \mathrm{CH}=\right), 1.66\left(\right.$ br s, $3 \mathrm{H}, \mathrm{CH}=\mathrm{C}\left(\mathrm{CH}_{3}\right)_{a}\left(\mathrm{CH}_{3}\right)_{b}, 1.58\left(\right.$ br s, $\left.3 \mathrm{H}, \mathrm{CH}=\mathrm{C}\left(\mathrm{CH}_{3}\right)_{a}\left(\mathrm{CH}_{3}\right)_{b}\right)$, and $1.44(\mathrm{~s}, 3 \mathrm{H}$, 
$\left.\mathrm{C}\left(\mathrm{CH}_{3}\right) \mathrm{CH}_{2} \mathrm{CH}_{2}\right)$.

${ }^{13} \mathbf{C}$ NMR $\left(125 \mathrm{MHz}, \mathrm{CDCl}_{3}\right): \delta 150.1,139.7,135.1,131.9,128.7,124.42,124.40,124.1,121.4,119.7,119.5$, $118.6,117.7,116.8,110.6,104.4,78.4,40.1,26.1,25.9,23.0,17.8$, and 16.3.

IR: 2922, 2865, 2844, 1456, 1055, 1033, 1014, 909, and $840 \mathrm{~cm}^{-1}$.

HRMS (ESI-TOF): Calcd for $\mathrm{C}_{23} \mathrm{H}_{25} \mathrm{NNaO}^{+}[\mathrm{M}+\mathrm{Na}]^{+}$requires 354.1828; found 354.1809.

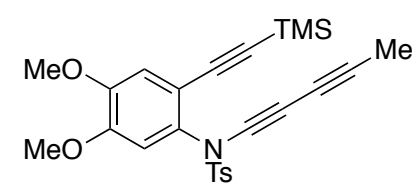

17

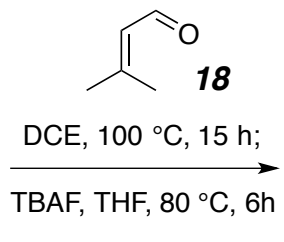

TBAF, THF, $80^{\circ} \mathrm{C}, 6 \mathrm{~h}$

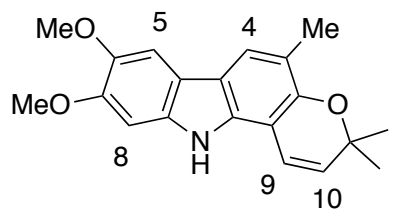

19

\section{Preparation of koenidine (19)}

Following General Procedure B, 17 (47 mg, $0.1 \mathrm{mmol})$, 3-methylbut-2-enal (18, $42 \mathrm{mg}, 0.5 \mathrm{mmol})$, and DCE (5 $\mathrm{mL})$ were used. The crude reaction mixture was concentrated and re-dissolved in THF $(1 \mathrm{~mL})$. TBAF $(0.2 \mathrm{~mL}, 1$ $\mathrm{M}$ in THF) was added slowly at room temperature. The vial was sealed with a Teflon-lined cap and placed in an $80{ }^{\circ} \mathrm{C}$ oil bath for $6 \mathrm{~h}$. The cooled reaction mixture was partitioned between $\mathrm{NH}_{4} \mathrm{Cl}$ (aq) and ethyl acetate. The organic layer was washed with brine, dried over anhydrous $\mathrm{Na}_{2} \mathrm{SO}_{4}$, filtered, and concentrated. The residue was purified by column chromatography (hexanes: ethyl acetate $=5: 1)$ to give koenidine $(19,22 \mathrm{mg}, 0.084 \mathrm{mmol}$, $84 \%$ ) as a pale-yellow oil.

${ }^{1}$ H NMR $\left(500 \mathrm{MHz}, \mathrm{CDCl}_{3}\right): \delta 7.70(\mathrm{br} \mathrm{s}, 1 \mathrm{H}$, carbazole $\mathrm{NH}), 7.55(\mathrm{~s}, 1 \mathrm{H}, \mathrm{ArH4}), 7.38(\mathrm{~s}, 1 \mathrm{H}, \mathrm{ArH5}), 6.91(\mathrm{~s}$, $1 \mathrm{H}, \mathrm{ArH8}), 6.60(\mathrm{~d}, J=9.7 \mathrm{~Hz}, 1 \mathrm{H}, H 9), 5.69(\mathrm{~d}, J=9.7 \mathrm{~Hz}, 1 \mathrm{H}, H 10), 3.98\left[\mathrm{~s}, 3 \mathrm{H}, \operatorname{Ar}\left(\mathrm{OCH}_{3}\right)_{a}\right], 3.94[\mathrm{~s}, 3 \mathrm{H}$, $\left.\operatorname{Ar}\left(\mathrm{OCH}_{3}\right)_{b}\right], 2.32\left(\mathrm{~d}, \mathrm{~J}=0.8 \mathrm{~Hz}, 3 \mathrm{H}\right.$, carbazole $\left.\mathrm{CH}_{3}\right)$, and $1.48\left(\mathrm{~s}, 6 \mathrm{H}, \mathrm{C}\left(\mathrm{CH}_{3}\right)_{2}\right)$.

${ }^{13}$ C NMR $\left(125 \mathrm{MHz}, \mathrm{CDCl}_{3}\right): \delta 149.0,148.5,144.7,135.0,134.3,129.8,120.4,118.6,117.5\left(2 \mathrm{xC}^{13}\right), 116.4$, $104.9,102.5,95.0,76.0,56.8,56.5,27.8$, and 16.3.

IR: $3424,3363,2973,2939,1644,1628,1494,1474,1444,1426,1276,1209,1161,1129,1118$, and $768 \mathrm{~cm}^{-1}$.

HRMS (ESI-TOF): Calcd for $\mathrm{C}_{20} \mathrm{H}_{21} \mathrm{NNaO}_{3}{ }^{+}$[M+Na $]^{+}$requires 346.1414; found 346.1415.

mp: $223-224{ }^{\circ} \mathrm{C}$ (lit., ${ }^{14} 224-225^{\circ} \mathrm{C}$ )

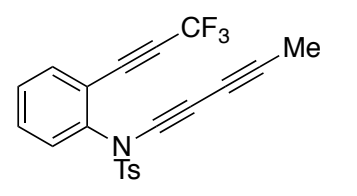

20<smiles>CC(C)(C)N1CC(=CC=O)C1</smiles>

21

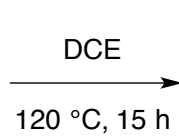

$120^{\circ} \mathrm{C}, 15 \mathrm{~h}$

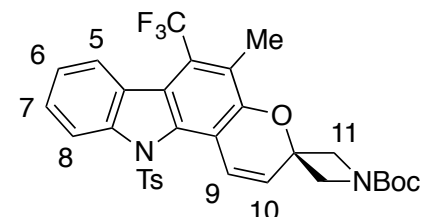

22

Preparation of tert-butyl 5'-methyl-11'-tosyl-6'-(trifluoromethyl)-11' $H$-spiro[azetidine-3,3'-pyrano[3,2a]carbazole]-1-carboxylate (22)

Compound 22 was prepared following General Procedure B using 20 (14 mg, 35 mol), tert-butyl 3-(2oxoethylidene)azetidine-1-carboxylate ${ }^{15}(35 \mathrm{mg}, 0.18 \mathrm{mmol})$, and DCE $(2 \mathrm{~mL})$. Purification by column chromatography (hexanes: ethyl acetate $=5: 1)$ gave product $22(15 \mathrm{mg}, 25 \mu \mathrm{mol}, 72 \%)$ as a pale yellow oil.

${ }^{1} \mathbf{H}$ NMR $\left(500 \mathrm{MHz}, \mathrm{CDCl}_{3}\right): \delta 8.15\left(\mathrm{ddd}, J=8.2,1.2,0.6 \mathrm{~Hz}, 1 \mathrm{H}, \mathrm{Ar} H_{8}\right), 7.80(\mathrm{dddq}, J=8.2,1.7,1.4,0.6 \mathrm{~Hz}$, $\left.1 \mathrm{H}, \mathrm{Ar} H_{5}\right), 7.38\left(\mathrm{ddd}, J=8.2,7.3,1.2 \mathrm{~Hz}, 1 \mathrm{H}, \mathrm{Ar} H_{7}\right.$ or $\left.\mathrm{Ar} H_{6}\right), 7.37$ (d, $\left.J=9.8 \mathrm{~Hz}, 1 \mathrm{H}, H_{9}\right), 7.27$ (ddd, $J=8.2$, 
7.3, $1.2 \mathrm{~Hz}, 1 \mathrm{H}, \mathrm{Ar} H_{7}$ or $\left.\mathrm{Ar} H_{6}\right), 6.85\left(\mathrm{~s}, 4 \mathrm{H}, \mathrm{ArSO}_{2} \mathrm{Ar} H_{o, m}\right), 6.12\left(\mathrm{~d}, J=9.8 \mathrm{~Hz}, 1 \mathrm{H}, H_{10}\right), 4.33(\mathrm{dd}, J=9.5,1.0$ $\left.\mathrm{Hz}, 2 \mathrm{H}, H_{1 l a}\right), 4.17\left(\mathrm{~d}, J=9.5,1.0 \mathrm{~Hz}, 2 \mathrm{H}, H_{l l b}\right), 2.50\left(\mathrm{q}, J=3.6 \mathrm{~Hz}, 3 \mathrm{H}, \mathrm{Ar}_{\text {carbazole }} \mathrm{CH}_{3}\right), 2.20\left(\mathrm{~s}, 3 \mathrm{H}\right.$, tosyl $\left.\mathrm{CH}_{3}\right)$, and $1.50\left[\mathrm{~s}, 9 \mathrm{H}, \mathrm{NCO}_{2} \mathrm{C}\left(\mathrm{CH}_{3}\right)_{3}\right]$.

${ }^{13}$ C NMR (125 MHz, $\left.\mathrm{CDCl}_{3}\right): \delta 156.5,150.1,145.1,141.9,137.0,131.1,130.0,128.8,128.2,127.15,127.11$, $126.2,124.9$ (q, $J=275.1 \mathrm{~Hz}), 124.2,123.6,123.4$ (q, $J=7.5 \mathrm{~Hz}), 123.4$ (q, $J=2.7 \mathrm{~Hz}), 122.0(\mathrm{q}, J=29.9 \mathrm{~Hz})$, 119.6, 116.8, 80.4, 74.1, 63.7 (br), 28.6, 21.6, and 12.8 (q, $J=4.6 \mathrm{~Hz})$.

IR: $2978,2885,1704,1391,1375,1324,1248,1176,1118,1083,1021$, and $759 \mathrm{~cm}^{-1}$.

HRMS (ESI-TOF): Calcd for $\mathrm{C}_{31} \mathrm{H}_{29} \mathrm{~F}_{3} \mathrm{~N}_{2} \mathrm{NaO}_{5} \mathrm{~S}^{+}[\mathrm{M}+\mathrm{Na}]^{+}$requires 621.1641; found 621.1656. 


\section{Computational methods and coordinates of structures}

DFT calculations were carried out using the Gaussian 09 software package. ${ }^{16}$ Geometries were optimized using the M06-2X functional developed by Truhlar et $\mathrm{al}^{17}$ and the double- $\zeta 6-31 \mathrm{G}(\mathrm{d})$ basis set. The SMD continuum solvation model $^{18}$ was used with 1,2-dichloroethane as the solvent during geometry optimization and frequency calculation. The "grid=ultrafine" option was applied during the numerical integrations. The harmonic vibrational frequency calculations were carried out using $298 \mathrm{~K}$ as the thermal correction for the free energies.

The following benzyne geometries were optimized:

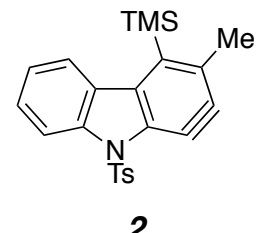

2

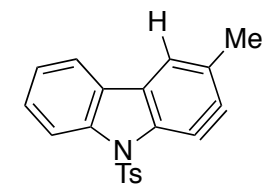

2-TMS<smiles>Cc1ccc2c(c1[AsH3])-c1ccccc1C2</smiles>

$2_{\mathrm{CH} 2}$<smiles>Cc1ccc2c(c1[N+](=O)[O-])-c1ccccc1C2</smiles>

2CH2-TMS

\section{Coordinates:}
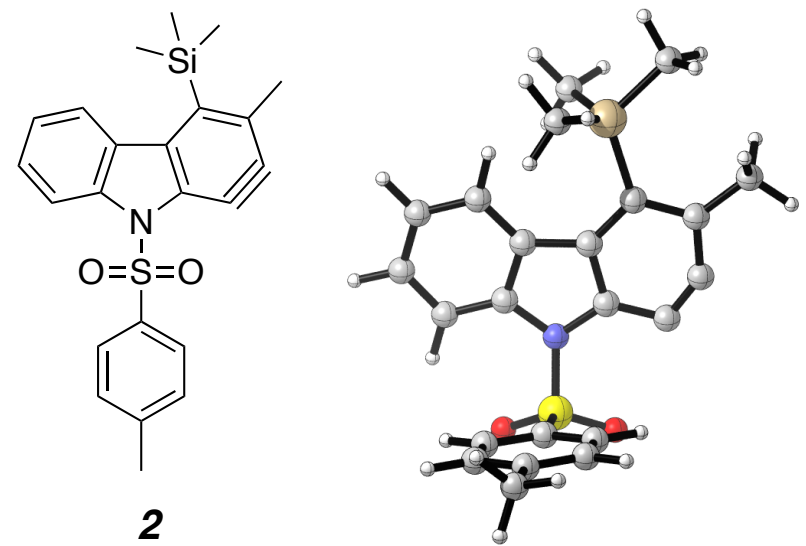

2

\begin{tabular}{|c|c|c|c|}
\hline Center & Atomic & Atomic & Coordinates (Angstroms) \\
\hline Number & Number & Type & $\begin{array}{lll}X & Y & Z\end{array}$ \\
\hline
\end{tabular}

$\begin{array}{rrrrrr}1 & 6 & 0 & -0.351992 & 3.794026 & 0.615937 \\ 2 & 6 & 0 & -0.980089 & 2.899438 & -0.239702 \\ 3 & 6 & 0 & -0.323447 & 1.701516 & -0.522051 \\ 4 & 6 & 0 & 0.946250 & 1.376750 & 0.012950 \\ 5 & 6 & 0 & 1.551668 & 2.313982 & 0.864353 \\ 6 & 6 & 0 & 0.902217 & 3.504039 & 1.162180 \\ 7 & 1 & 0 & -0.842800 & 4.732315 & 0.854133 \\ 8 & 1 & 0 & -1.941100 & 3.128562 & -0.681564 \\ 9 & 1 & 0 & 2.522452 & 2.131673 & 1.304851 \\ 10 & 1 & 0 & 1.380133 & 4.218009 & 1.825139 \\ 11 & 6 & 0 & 1.320417 & 0.044240 & -0.486674 \\ 12 & 6 & 0 & 2.459420 & -0.800928 & -0.300155 \\ 13 & 6 & 0 & 0.218703 & -0.362398 & -1.298469 \\ 14 & 6 & 0 & 2.491399 & -2.094612 & -0.900321\end{array}$




\begin{tabular}{|c|c|c|c|c|c|}
\hline 15 & 6 & $\theta$ & 0.282607 & -1.619596 & -1.868153 \\
\hline 16 & 6 & 0 & 1.320493 & -2.259978 & -1.608019 \\
\hline 17 & 16 & 0 & -2.363640 & 0.325931 & -1.762754 \\
\hline 18 & 8 & 0 & -2.973800 & 1.615780 & -2.031521 \\
\hline 19 & 8 & 0 & -2.314250 & -0.699487 & -2.786386 \\
\hline 20 & 6 & 0 & 3.581543 & -3.126513 & -0.805046 \\
\hline 21 & 1 & 0 & 4.513844 & -2.771360 & -1.251156 \\
\hline 22 & 1 & 0 & 3.784196 & -3.402242 & 0.232710 \\
\hline 23 & 1 & 0 & 3.275307 & -4.027721 & -1.339476 \\
\hline 24 & 14 & 0 & 3.930559 & -0.159825 & 0.746315 \\
\hline 25 & 6 & 0 & 5.455848 & -1.256823 & 0.851146 \\
\hline 26 & 1 & 0 & 5.293510 & -2.208480 & 1.364013 \\
\hline 27 & 1 & 0 & 5.921561 & -1.452510 & -0.118877 \\
\hline 28 & 1 & 0 & 6.182213 & -0.686756 & 1.446981 \\
\hline 29 & 6 & 0 & 4.555353 & 1.427282 & -0.065979 \\
\hline 30 & 1 & 0 & 5.329011 & 1.154883 & -0.793608 \\
\hline 31 & 1 & 0 & 3.788202 & 1.994487 & -0.599398 \\
\hline 32 & 1 & 0 & 5.021141 & 2.093602 & 0.669932 \\
\hline 33 & 6 & $\theta$ & 3.323352 & 0.023082 & 2.524982 \\
\hline 34 & 1 & 0 & 2.277190 & 0.328159 & 2.613031 \\
\hline 35 & 1 & 0 & 3.425318 & -0.947205 & 3.025625 \\
\hline 36 & 1 & $\theta$ & 3.936800 & 0.742337 & 3.080738 \\
\hline 37 & 7 & 0 & -0.748422 & 0.651350 & -1.361950 \\
\hline 38 & 6 & 0 & -4.056057 & -1.411311 & 2.077625 \\
\hline 39 & 6 & $\theta$ & -4.166387 & -0.040838 & 1.809085 \\
\hline 40 & 6 & 0 & -3.667196 & 0.501392 & 0.632345 \\
\hline 41 & 6 & 0 & -3.052298 & -0.350680 & -0.284609 \\
\hline 42 & 6 & 0 & -2.923637 & -1.717361 & -0.048735 \\
\hline 43 & 6 & $\theta$ & -3.430484 & -2.236498 & 1.137462 \\
\hline 44 & 1 & 0 & -4.653938 & 0.607750 & 2.531793 \\
\hline 45 & 1 & $\theta$ & -3.764410 & 1.561376 & 0.420247 \\
\hline 46 & 1 & $\theta$ & -2.445681 & -2.360477 & -0.781221 \\
\hline 47 & 1 & 0 & -3.342582 & -3.300854 & 1.334957 \\
\hline 48 & 6 & 0 & -4.623303 & -1.980414 & 3.349377 \\
\hline 49 & 1 & 0 & -4.315304 & -1.387257 & 4.215695 \\
\hline 50 & 1 & $\theta$ & -5.718515 & -1.969586 & 3.319708 \\
\hline 51 & 1 & 0 & -4.299055 & -3.012638 & 3.501134 \\
\hline
\end{tabular}




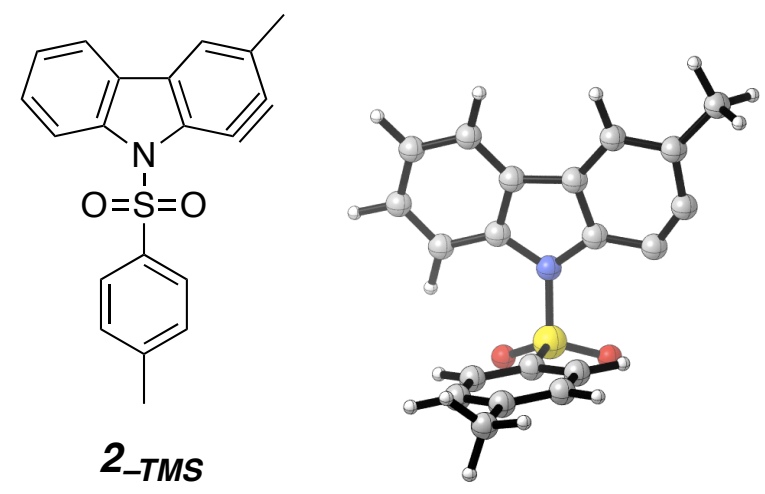

$\begin{array}{llll}\text { Center } & \text { Atomic } & \text { Atomic } & \text { Coordinates (Angstroms) } \\ \text { Number } & \text { Number } & \text { Type } & \mathrm{X}\end{array}$

$\begin{array}{rrrrrr}1 & 6 & 0 & -0.528750 & 3.796261 & -0.891991 \\ 2 & 6 & 0 & -0.096557 & 2.909228 & 0.088350 \\ 3 & 6 & 0 & -0.747840 & 1.678874 & 0.170343 \\ 4 & 6 & 0 & -1.807585 & 1.339334 & -0.697286 \\ 5 & 6 & 0 & -2.231879 & 2.253946 & -1.663854 \\ 6 & 6 & 0 & -1.584304 & 3.478446 & -1.757571 \\ 7 & 1 & 0 & -0.037900 & 4.760493 & -0.979256 \\ 8 & 1 & 0 & 0.705747 & 3.171087 & 0.766798 \\ 9 & 1 & 0 & -3.052342 & 2.004337 & -2.329899 \\ 10 & 1 & 0 & -1.899838 & 4.198987 & -2.505332 \\ 11 & 6 & 0 & -2.247907 & -0.002760 & -0.353609 \\ 12 & 6 & 0 & -3.250410 & -0.839959 & -0.879467 \\ 13 & 6 & 0 & -1.424945 & -0.426268 & 0.719264 \\ 14 & 6 & 0 & -3.483148 & -2.140610 & -0.391532 \\ 15 & 6 & 0 & -1.667677 & -1.707538 & 1.184544 \\ 16 & 6 & 0 & -2.573902 & -2.361705 & 0.633685 \\ 17 & 16 & 0 & 0.920956 & 0.293751 & 1.870422 \\ 18 & 8 & 0 & 1.408315 & 1.584394 & 2.323289 \\ 19 & 8 & 0 & 0.623400 & -0.767585 & 2.812955 \\ 20 & 6 & 0 & -4.536768 & -3.063797 & -0.921869 \\ 21 & 1 & 0 & -5.253950 & -3.328017 & -0.138836 \\ 22 & 1 & 0 & -5.079189 & -2.589011 & -1.742465 \\ 23 & 1 & 0 & -4.092651 & -3.993413 & -1.290407 \\ 24 & 7 & 0 & -0.538953 & 0.606549 & 1.072695 \\ 25 & 6 & 0 & 1.988611 & -0.324455 & 0.607338 \\ 26 & 6 & 0 & 1.947287 & -1.681947 & 0.297917 \\ 27 & 6 & 0 & 2.810183 & 0.565190 & -0.084068 \\ 28 & 6 & 0 & 2.757053 & -2.152745 & -0.729822 \\ 29 & 1 & 0 & 1.302426 & -2.355543 & 0.854060 \\ 30 & 6 & 0 & 3.610942 & 0.070791 & -1.105030 \\ 31 & 1 & 0 & 2.829093 & 1.617086 & 0.182685 \\ 32 & 6 & 0 & 3.595477 & -1.288930 & -1.441825\end{array}$




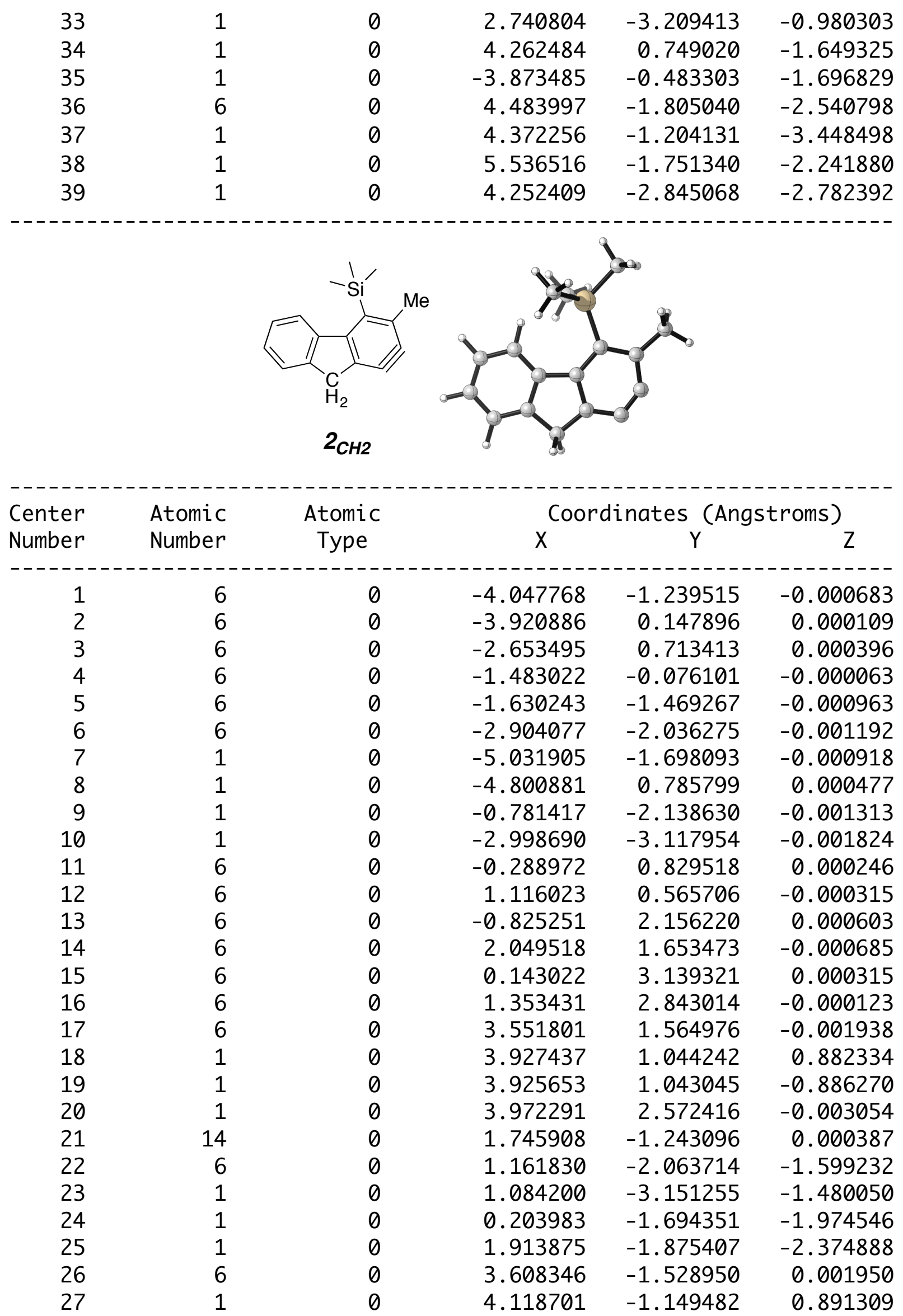




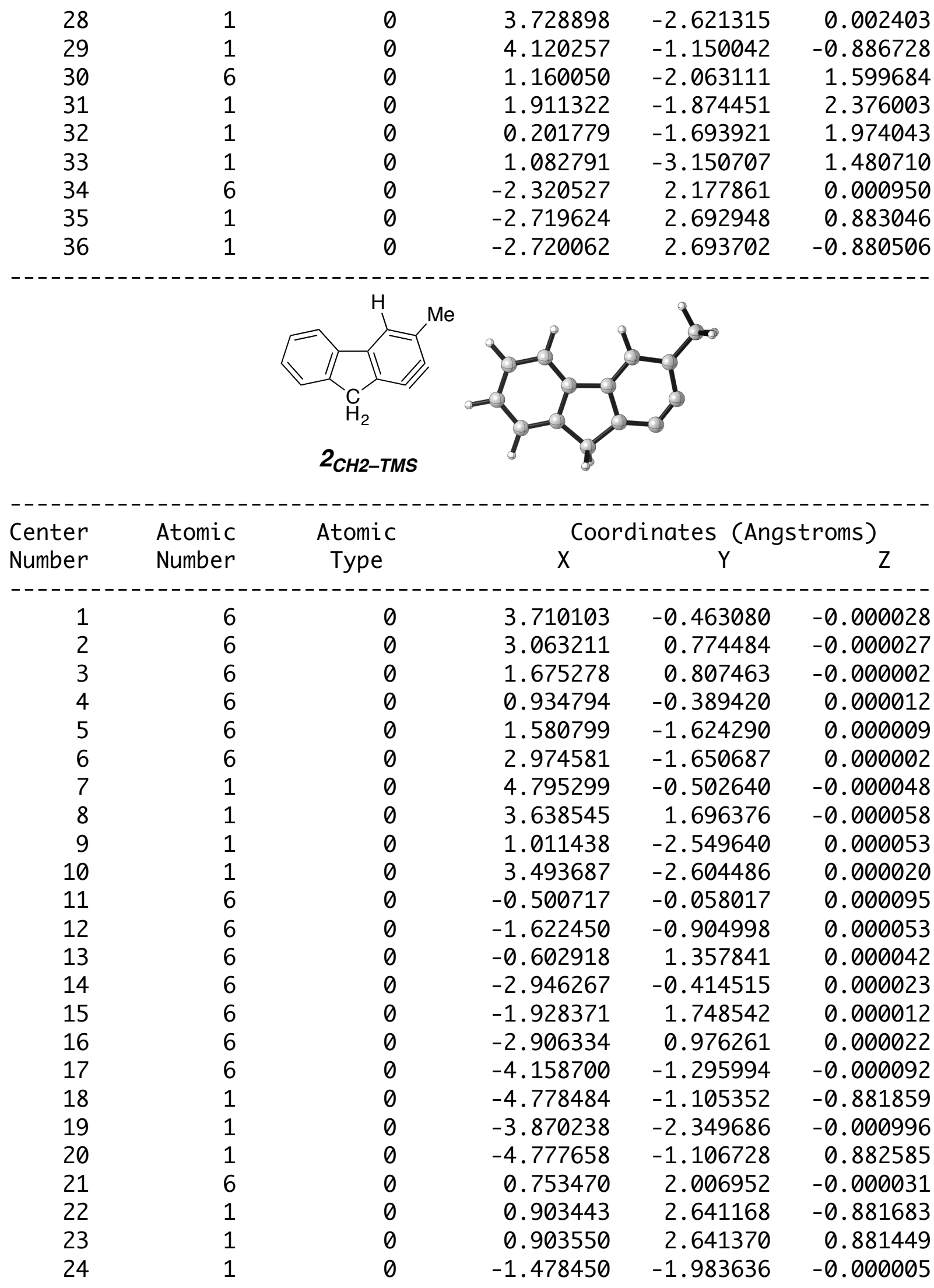




\section{References and Notes}

1. Hoye, T. R.; Zhao, H. J. Org. Chem. 2002, 67, 4014-4016.

2. Laroche, C.; Li, J.; Freyer, M. W.; Kerwin, S. M. J. Org. Chem. 2008, 73, 6462-6465.

3. Alayrac, C.; Schollmeyerb, D.; Witulski, B. Chem. Commun. 2009, 1464-1466.

4. a) Procedure for preparation of bromopropyne ${ }^{4 \mathrm{~b}}$ :

"A $2 \mathrm{~L}$ three-neck round-bottom flask containing a magnetic stir bar was charged with a solution of $\mathrm{KOH}$ ( $85 \%$ tech. grade, $260 \mathrm{~g}, 3.94 \mathrm{~mol}$ ) in $250 \mathrm{~mL}$ of water, and the flask was placed in an ice-salt bath. The flask was fitted with a thermocouple, a pressure equalizing dropping funnel, and a rubber septum. When the $\mathrm{KOH}$ solution had cooled to $-3{ }^{\circ} \mathrm{C}$ (internal temperature), bromine ( $40 \mathrm{~mL}, 0.78 \mathrm{~mol}$, ca. 1.5 equiv) was added dropwise to the stirred solution at such a rate so that the internal temperature did not rise above $0{ }^{\circ} \mathrm{C}$. A precipitate formed over the course of the addition. After the addition was complete, the yellow slurry was stirred for an additional $30 \mathrm{~min}$ at $0{ }^{\circ} \mathrm{C}$.

A $250 \mathrm{~mL}$ Erlenmeyer flask containing a ground-glass joint was charged with $150 \mathrm{~mL}$ of hexanes and the flask was cooled in a dry ice-acetone bath. Gaseous propyne (bp $-23{ }^{\circ} \mathrm{C}, 95 \%$, ca. $30 \mathrm{~mL}, 0.50 \mathrm{~mol}$ ) was then slowly introduced via an 18 -gauge syringe needle into the headspace of the flask. Condensation is more efficient if the needle tip is close to the surface of the cold hexane. Condensation was allowed to continue until the total volume of the solution had grown to ca. $180 \mathrm{~mL}$. It is advisable to attach a bubbler to the headspace to ensure that the gas is not being introduced too rapidly.

The addition funnel on $2 \mathrm{~L}$ the three-neck flask was replaced by a Dewar condenser filled with a dry iceacetone mixture. The hexanes solution of propyne, still at $-78^{\circ} \mathrm{C}$, was added slowly to the aqueous $\mathrm{KOBr}$ solution, still maintained at $\leq 0{ }^{\circ} \mathrm{C}$, via cannula over approximately $1 \mathrm{~h}$. Depending on the rate of addition, the internal temperature of the reaction mixture may or may not increase. The reaction mixture was allowed to warm to room temperature, using internal temperature monitoring to guide the intermittent use of the cooling bath. As the mixture warmed, propyne reflux was observed, and the rate or reflux qualitatively indicated the progress of reaction. After the cessation of propyne reflux (ca. $2 \mathrm{~h}$ ), the reaction mixture was transferred to a $2 \mathrm{~L}$ separatory funnel. The aqueous layer was drained and combined with brine $(100 \mathrm{~mL})$ to minimize emulsion formation. The combined aqueous layers were extracted with hexanes $(2 \times 50 \mathrm{~mL})$, and the combined organic layers were washed with brine $(100 \mathrm{~mL})$, dried over $\mathrm{Na}_{2} \mathrm{SO}_{4}$, and filtered to give a dried stock solution of 1-bromopropyne (ca. $300 \mathrm{~mL}$ total volume). Caution: neat 1-bromopropyne (reported bp $64^{\circ} \mathrm{C}$ ) has been reported to ignite upon exposure to oxygen; ${ }^{4 \mathrm{c}}$ hence, we have opted to titer the hexanes solution by ${ }^{1} \mathrm{H}$ NMR analysis of an aliquot and use that solution directly for subsequent coupling reactions. The concentration of the stock solution described here was judged to be $27 \mathrm{wt} \%$. Such solutions have been stored multiple times in a freezer $\left(\right.$ ca. $-20^{\circ} \mathrm{C}$ ) for months with no obvious loss in titer (NMR) or discoloration." $4 \mathrm{~b}$

b) Chen, J.; Palani, V.; Hoye, T. R. J. Am. Chem. Soc. 2016, 138, 4318-4321.

c) Brandsma, L.; Verkruijsse, H. D. Synthesis 1990, 984-985.

5. Ohta, Y.; Chiba, H.; Oishi, S.; Fujii, N.; Ohno, H. J. Org. Chem. 2009, 74, 7052-7058.

6. Kitamura, T.; Morshed, M. H.; Tsukada, S.; Miyazaki, Y.; Iguchi, N.; Inoue, D. J. Org. Chem. 2011, 76, $8117-8120$.

7. Li, L.-S.; Wu, Y.-L. Tetrahedron Lett. 2002, 43, 2427-2430.

8. Yoo, W.-J.; Allen, A.; Villeneuve, K.; Tam, W. Org. Lett. 2005, 7, 5853-5856.

9. De Leon, P.; Egbertson, M.; Hills, I. D.; Johnson, A. W.; Machacek, M. Quinolinone PDE2 inhibitors. U.S. Patent 2456440 B1, August 19, 2015. 
10. Tresse, C.; Guissart, C.; Schweizer, S.; Bouhoute, Y.; Chany, A.-C.; Goddard, M.-L.; Blanchard, N.; Evano, G. Adv. Synth. Catal. 2014, 356, 2051-2060.

11. For example, a) Zhang, C.; Sun, P. J. Org. Chem. 2014, 79, 8457-8461 vs. b) Panda, N.; Jena, A. K.; Mohapatra, S.; Rout, S. R. Tetrahedron Lett. 2011, 52, 1924-1927.

12. Tachibana, Y.; Kikuzaki, H.; Lajis, N. H.; Nakatani, N. J. Agric. Food Chem. 2001, 49, 55894-5594.

13. Patel, O. P. S.; Mishra, A.; Maurya, R.; Saini, D.; Pandey, J.; Taneja, I.; Raju, K. S. R.; Kanojiya, S.; Shukla, S. K.; Srivastava, M. N.; Wahajuddin, M.; Tamrakar, A. K.; Srivastava, A. K.; Yadav, P. P. J. Nat. Prod. 2016, 79, 1276-1284.

14. Kureel, S. P.; Kapil, R. S.; Popli, S. P. Experientia 1969, 25, 790-791.

15. Burkhard, J. A.; Guérot, C.; Knust, H.; Rogers-Evans, M.; Carreira, E. M. Org. Lett. 2010, 12, 1944-1947.

16. M. J. Frisch, G. W. Trucks, H. B. Schlegel, G. E. Scuseria, M. A. Robb, J. R. Cheeseman, G. Scalmani, V. Barone, B. Mennucci, G. A. Petersson, H. Nakatsuji, M. Caricato, X. Li, H. P. Hratchian, A. F. Izmaylov, J. Bloino, G. Zheng, J. L. Sonnenberg, M. Hada, M. Ehara, K. Toyota, R. Fukuda, J. Hasegawa, M. Ishida, T. Nakajima, Y. Honda, O. Kitao, H. Nakai, T. Vreven, J. A. Montgomery, Jr., J. E. Peralta, F. Ogliaro, M. Bearpark, J. J. Heyd, E. Brothers, K. N. Kudin, V. N. Staroverov, R. Kobayashi, J. Normand, K. Raghavachari, A. Rendell, J. C. Burant, S. S. Iyengar, J. Tomasi, M. Cossi, N. Rega, J. M. Millam, M. Klene, J. E. Knox, J. B. Cross, V. Bakken, C. Adamo, J. Jaramillo, R. Gomperts, R. E. Stratmann, O. Yazyev, A. J. Austin, R. Cammi, C. Pomelli, J. W. Ochterski, R. L. Martin, K. Morokuma, V. G. Zakrzewski, G. A. Voth, P. Salvador, J. J. Dannenberg, S. Dapprich, A. D. Daniels, Ö. Farkas, J. B. Foresman, J. V. Ortiz, J. Cioslowski, and D. J. Fox. Gaussian 09, revision D.01; Gaussian, Inc.: Wallingform, CT, 2009.

17. Zhao, Y.; Truhlar, D. G. The M06 suite of density functionals for main group thermochemistry, thermochemical kinetics, noncovalent interactions, excited states, and transition elements: Two new functionals and systematic testing of four M06-class functionals and 12 other functionals. Theor. Chem. Acc. 2008, 120, 215-241.

18. Marenich, A. V.; Cramer, C. J.; and Truhlar, D. G. Universal Solvation Model Based on Solute Electron Density and on a Continuum Model of the Solvent Defined by the Bulk Dielectric Constant and Atomic Surface Tensions. J. Phys. Chem. B 2009, 113, 6378-6396. 


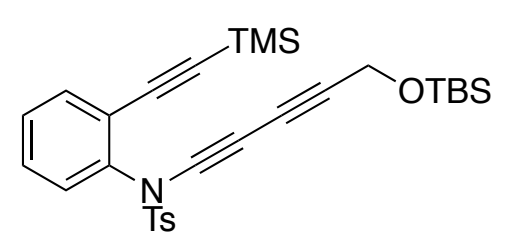

4-TMS/CH2OTBS

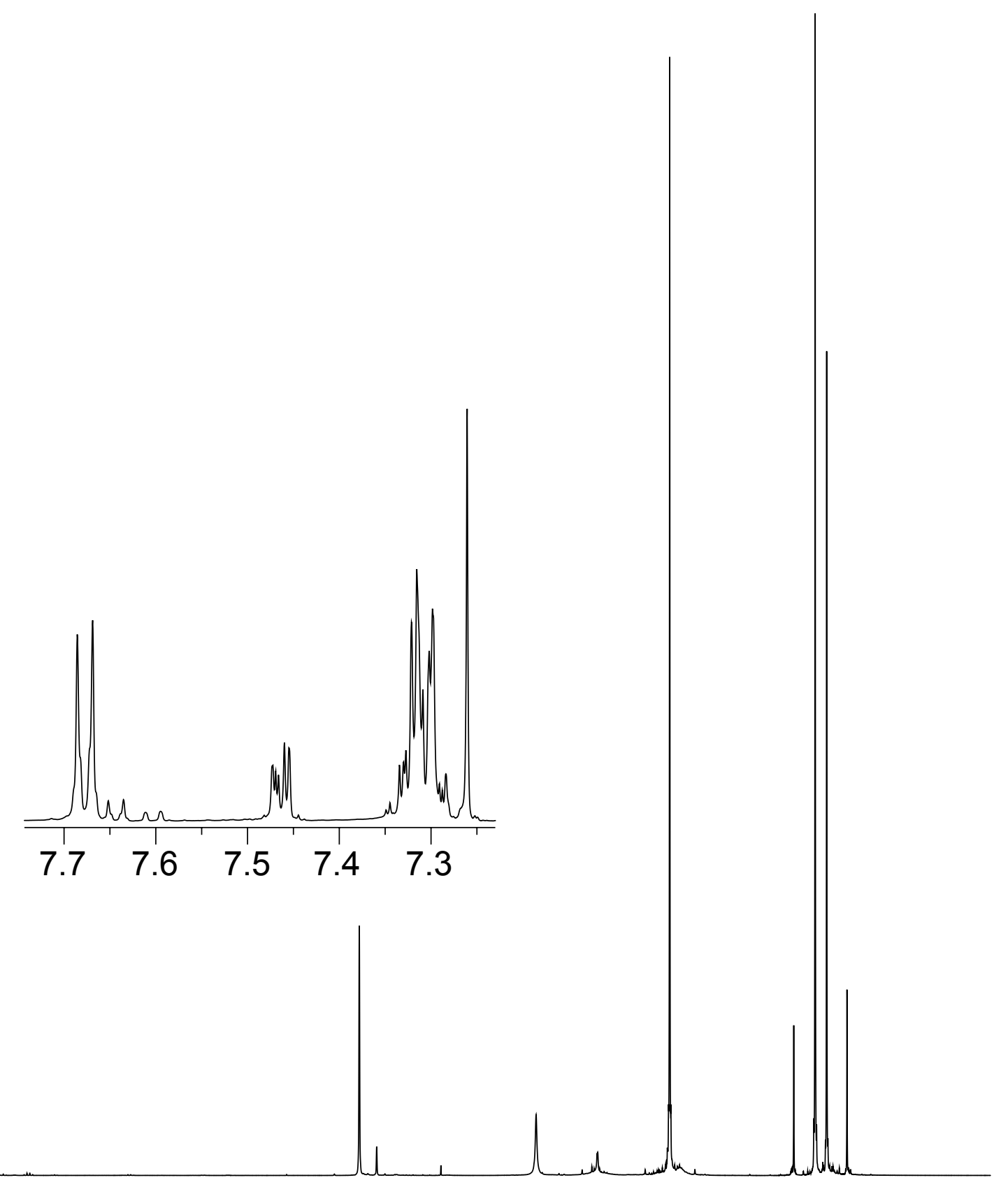

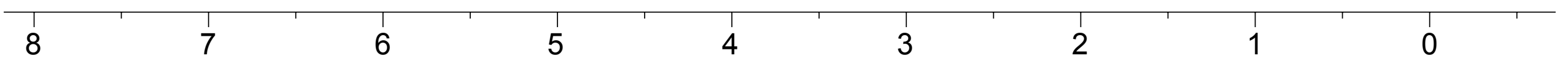




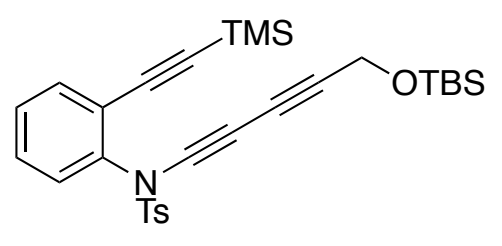

\section{4-TMS/CH2OTBS}

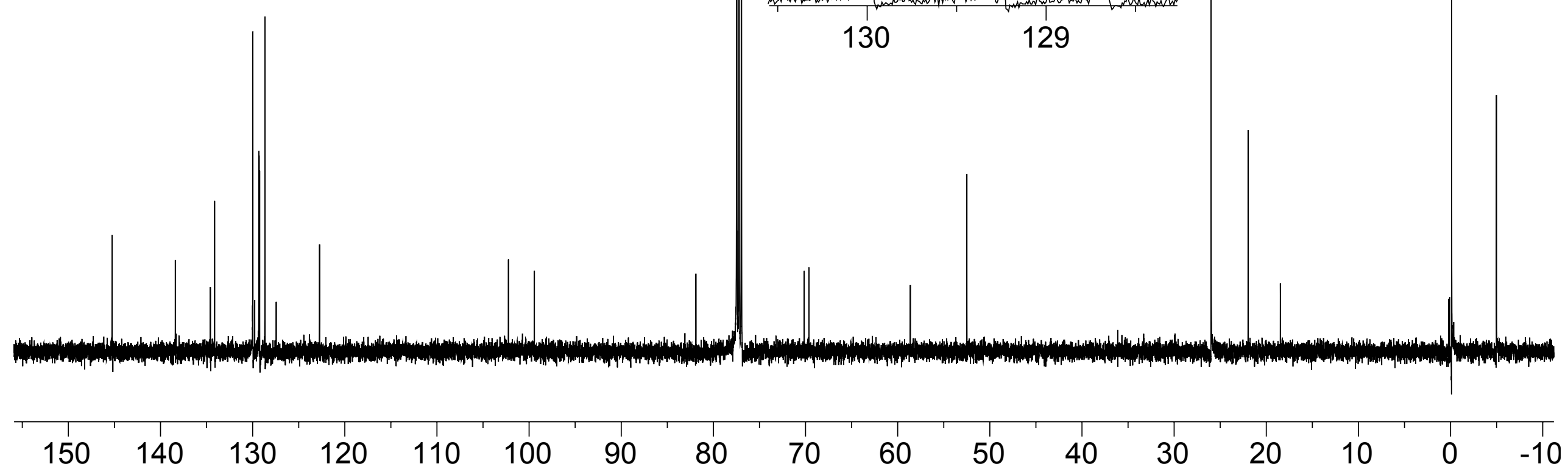



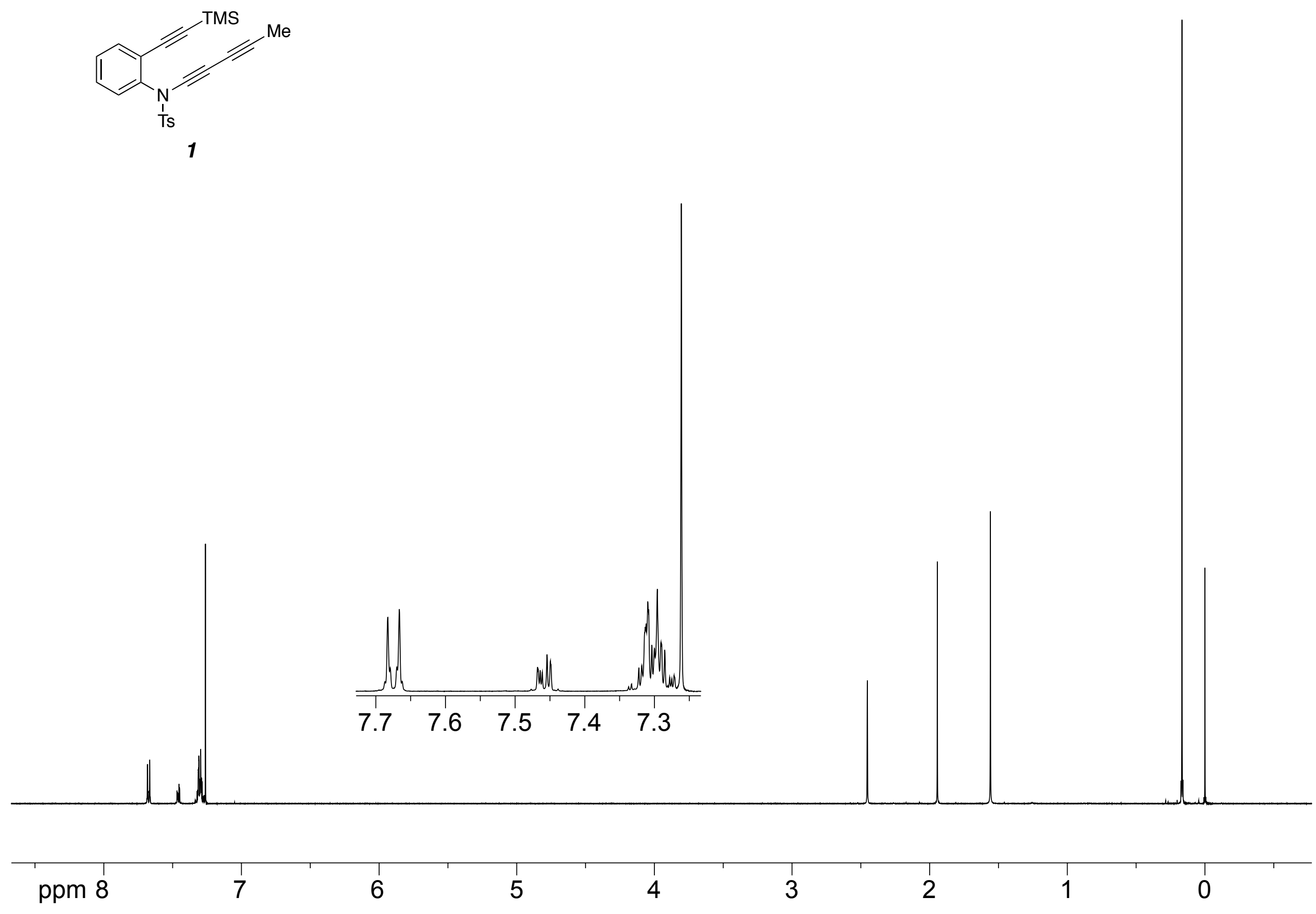

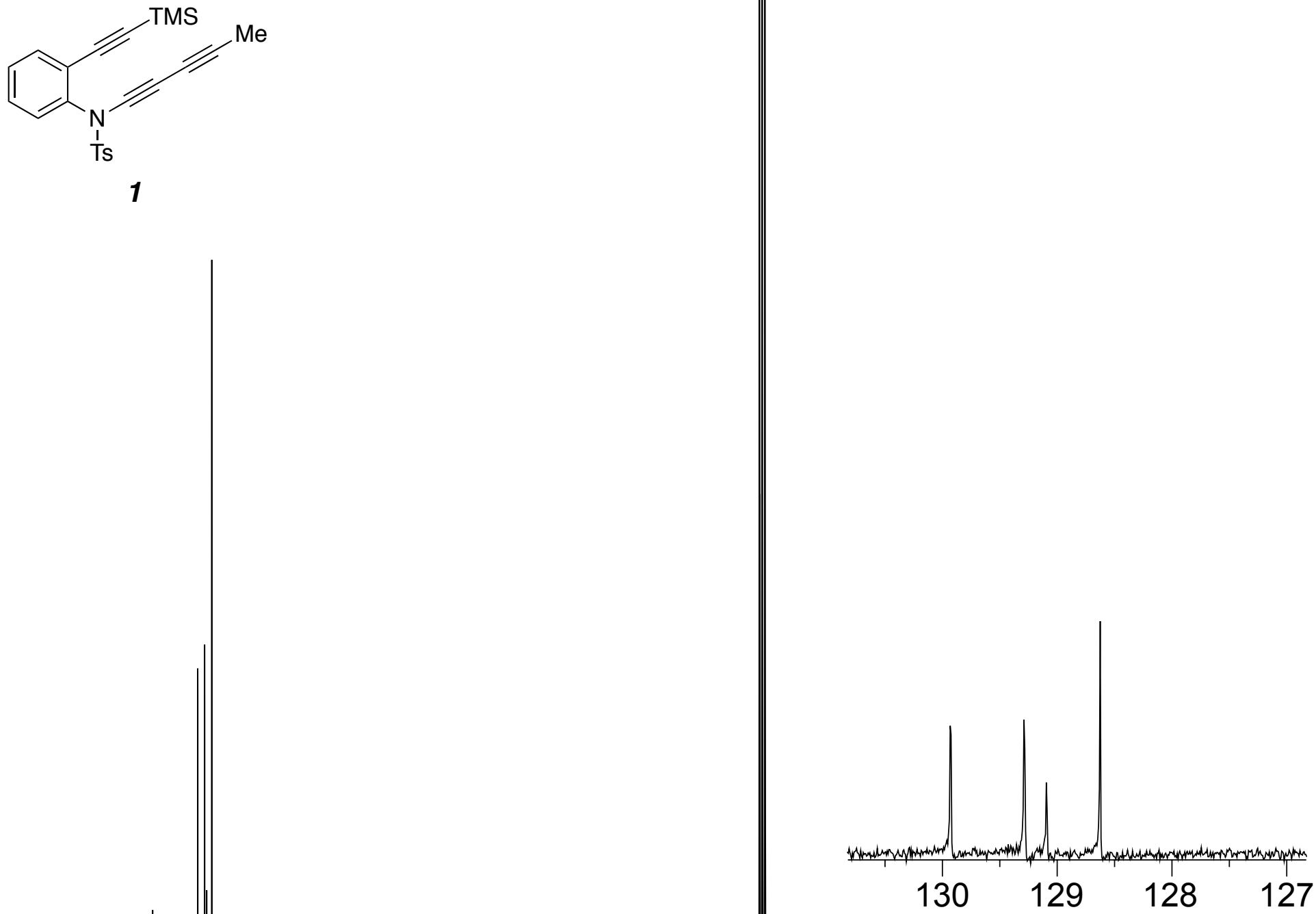

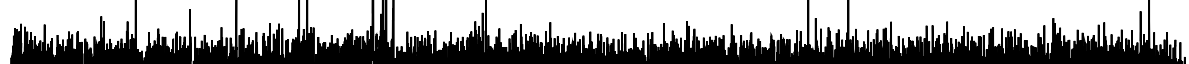

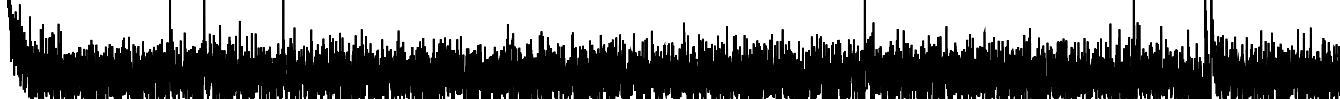
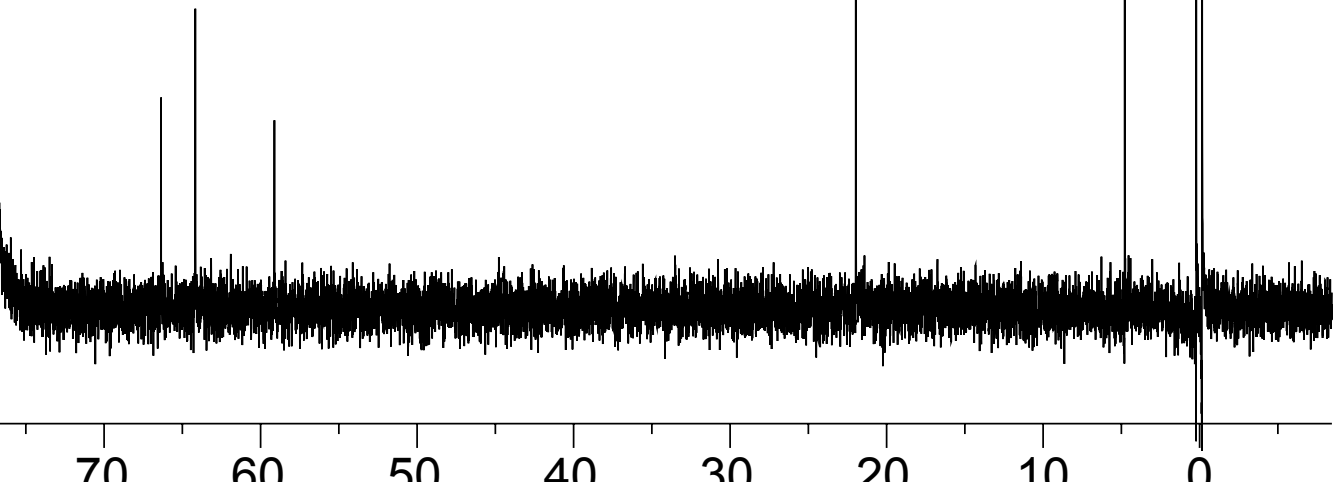


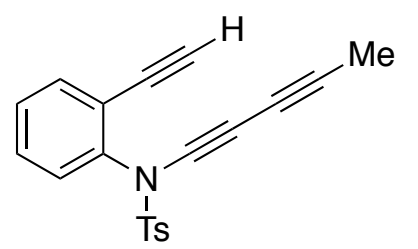

\section{4-H/Me}
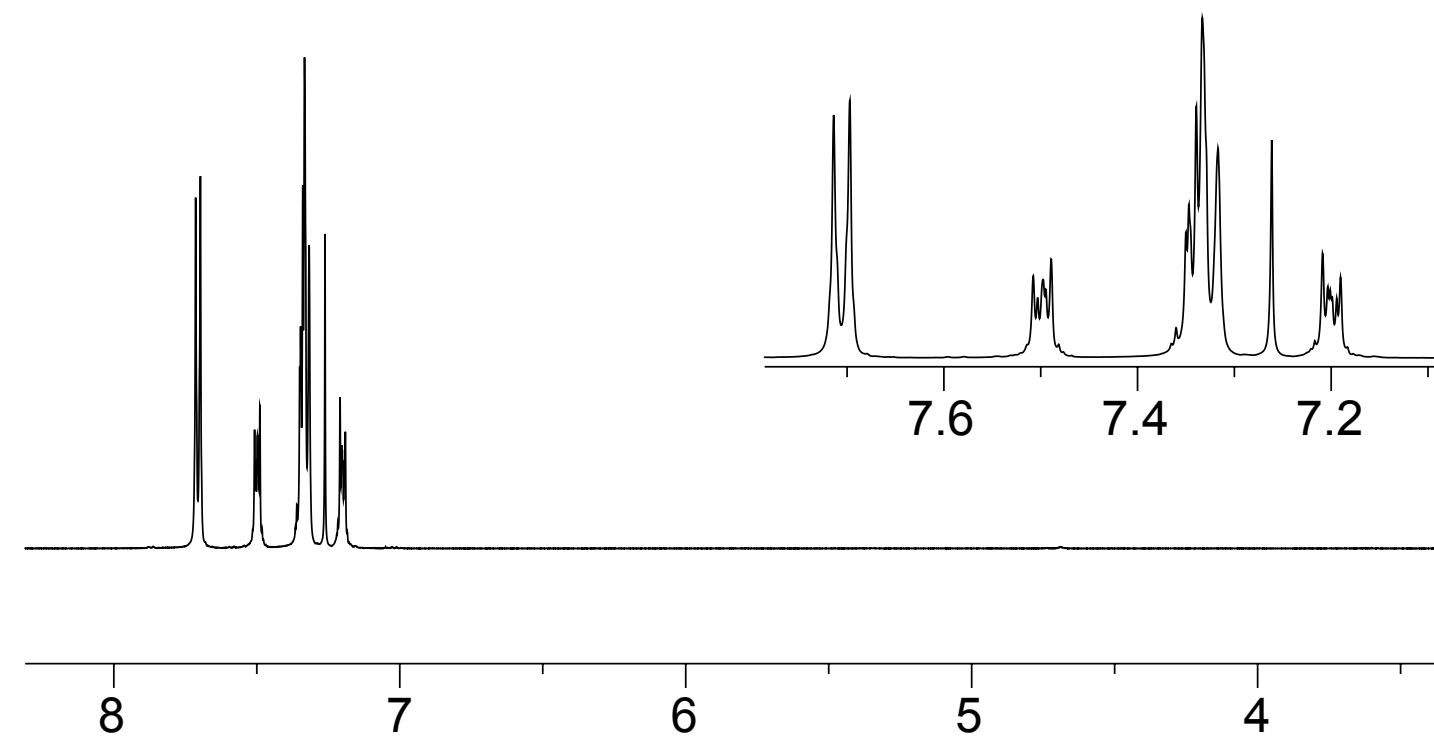

6

5

4

3

2

1 


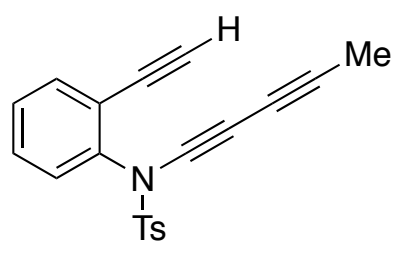

4-H/Me

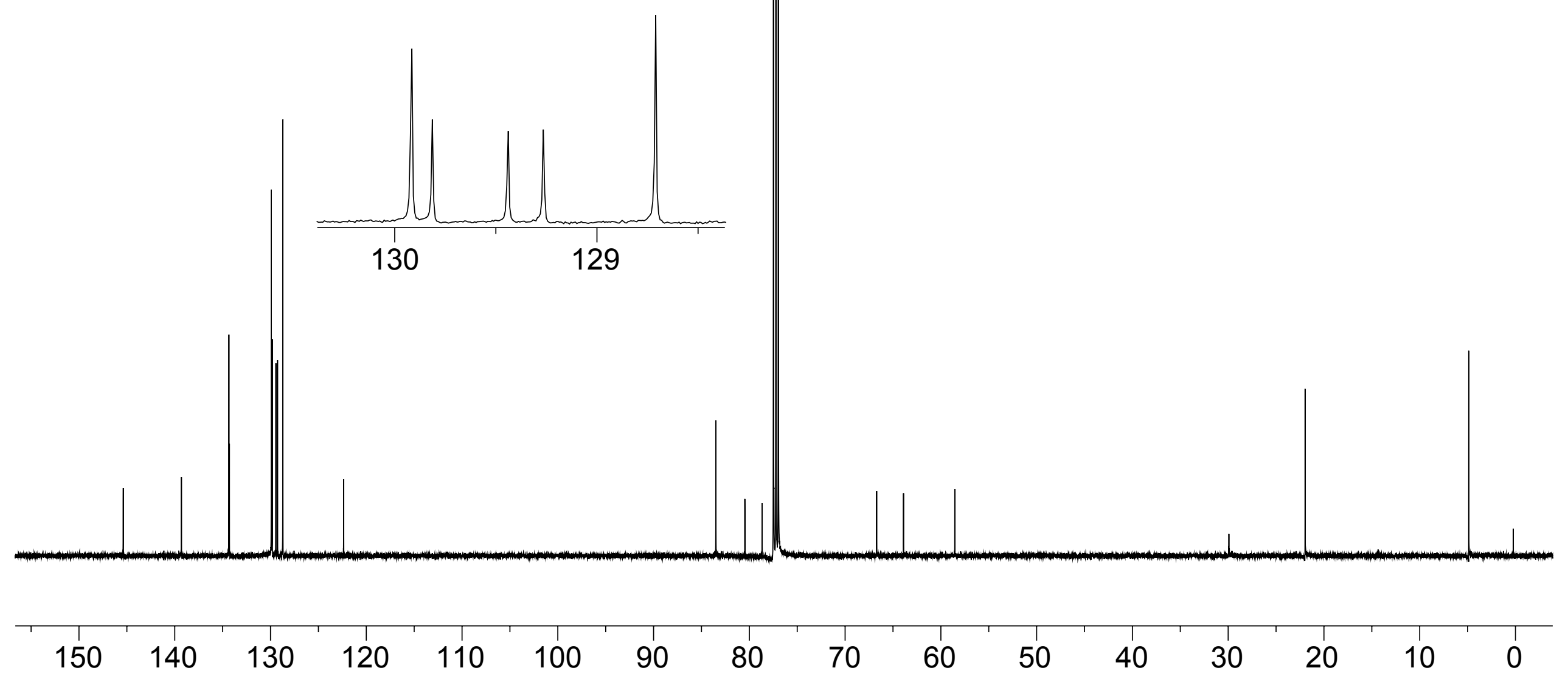



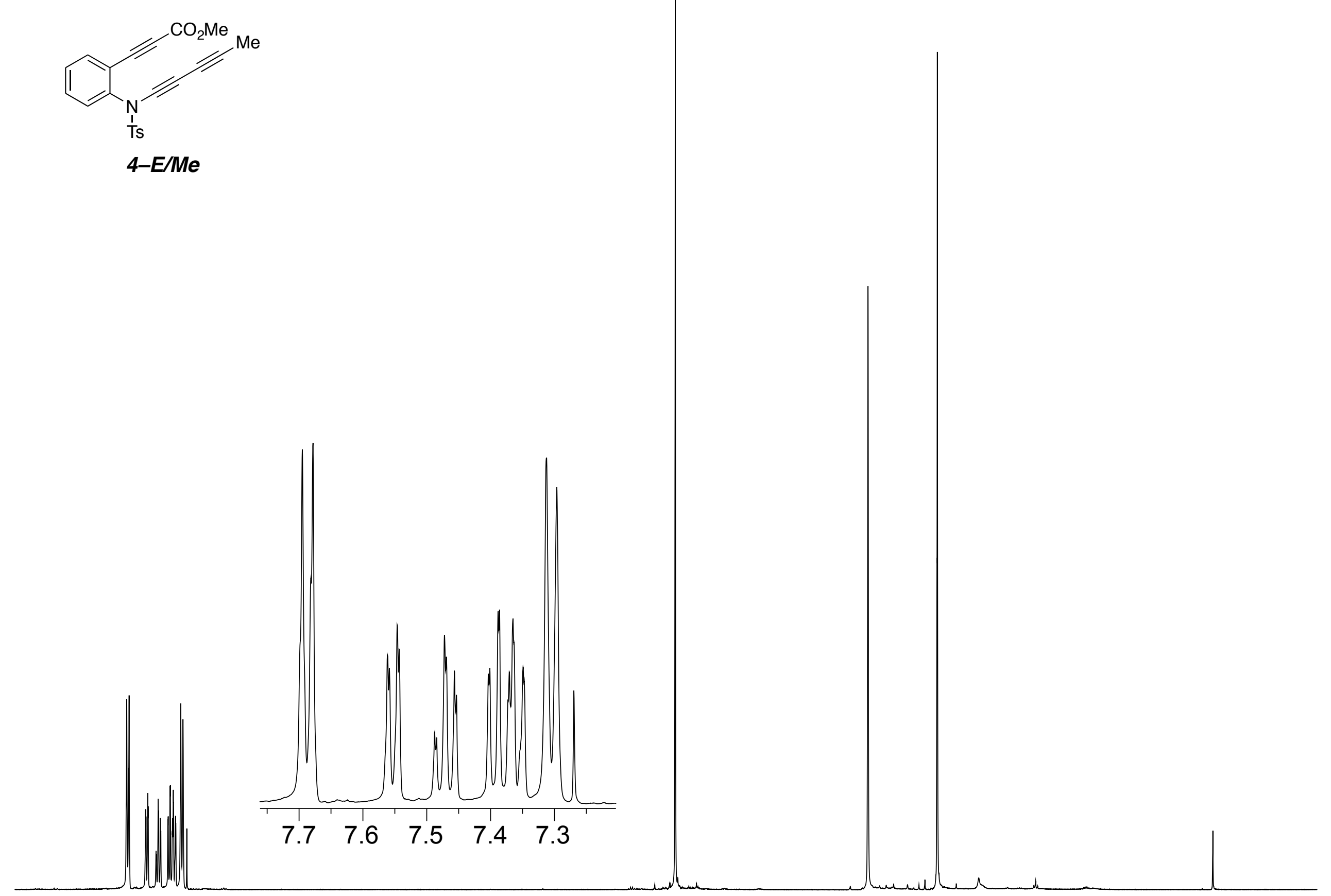


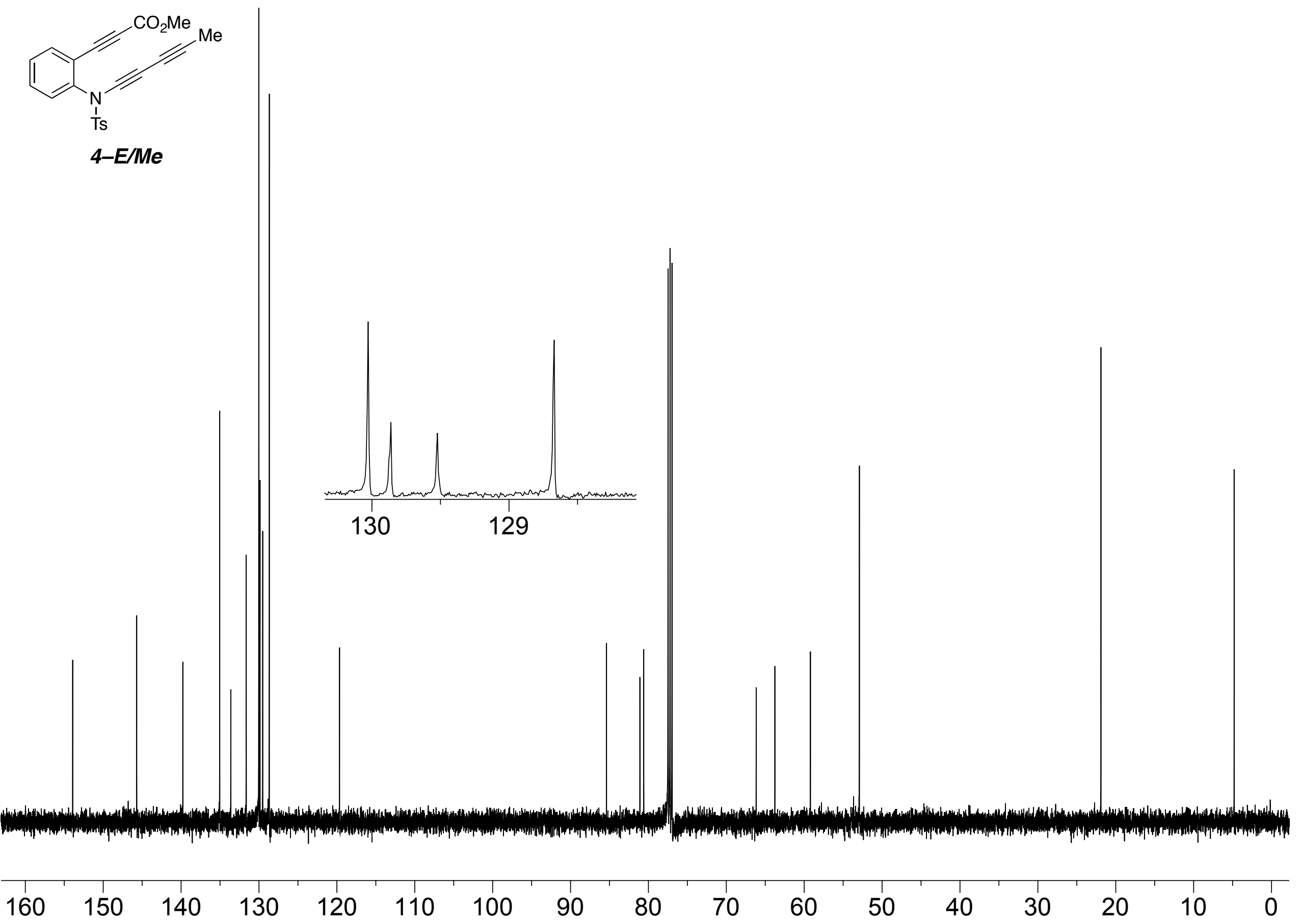




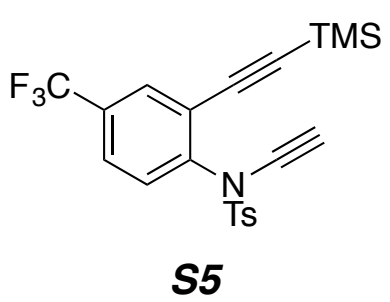

|ull

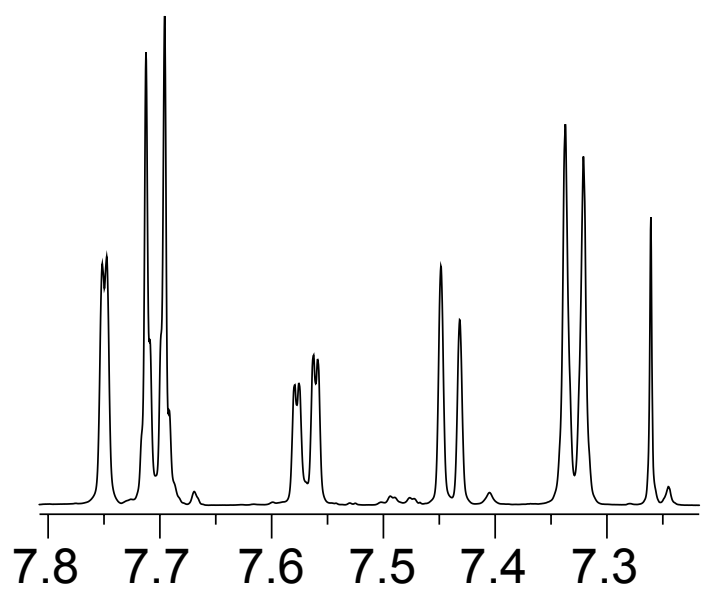




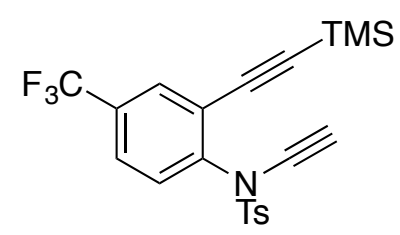

S5
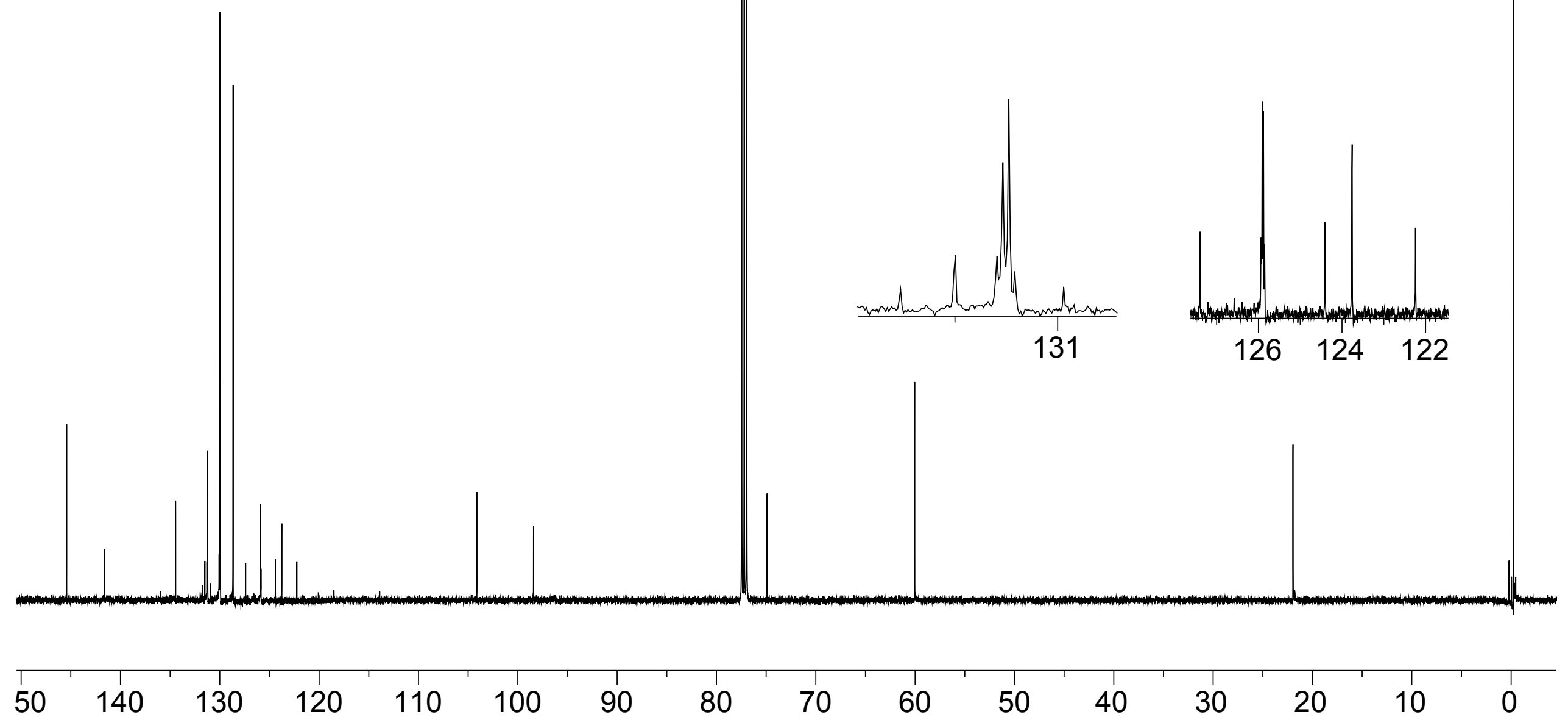


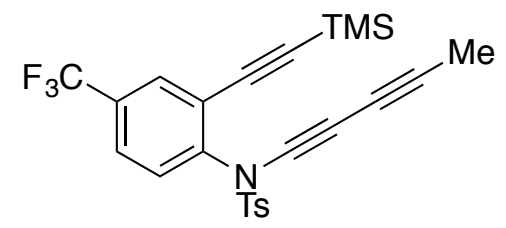

\section{4-(CF3)TMS/Me}
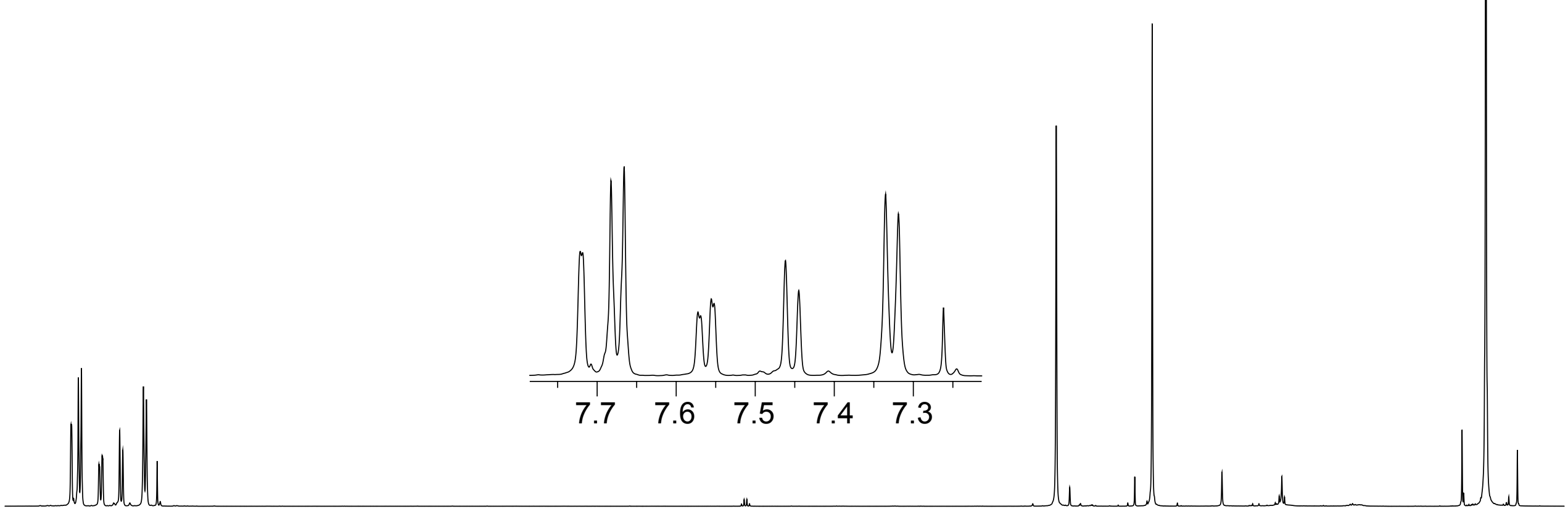

\begin{tabular}{l|l|l|l|l|l|l|l|l|l|}
\hline 8 & 7 & 6 & 5 & 4 & 3 & 2 & 1 & 0
\end{tabular}




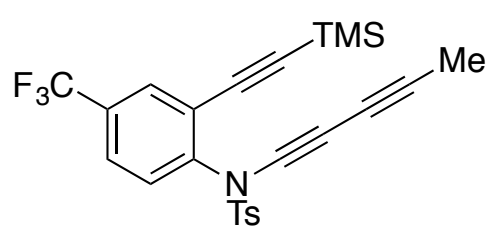

4-(CF3)TMS/Me

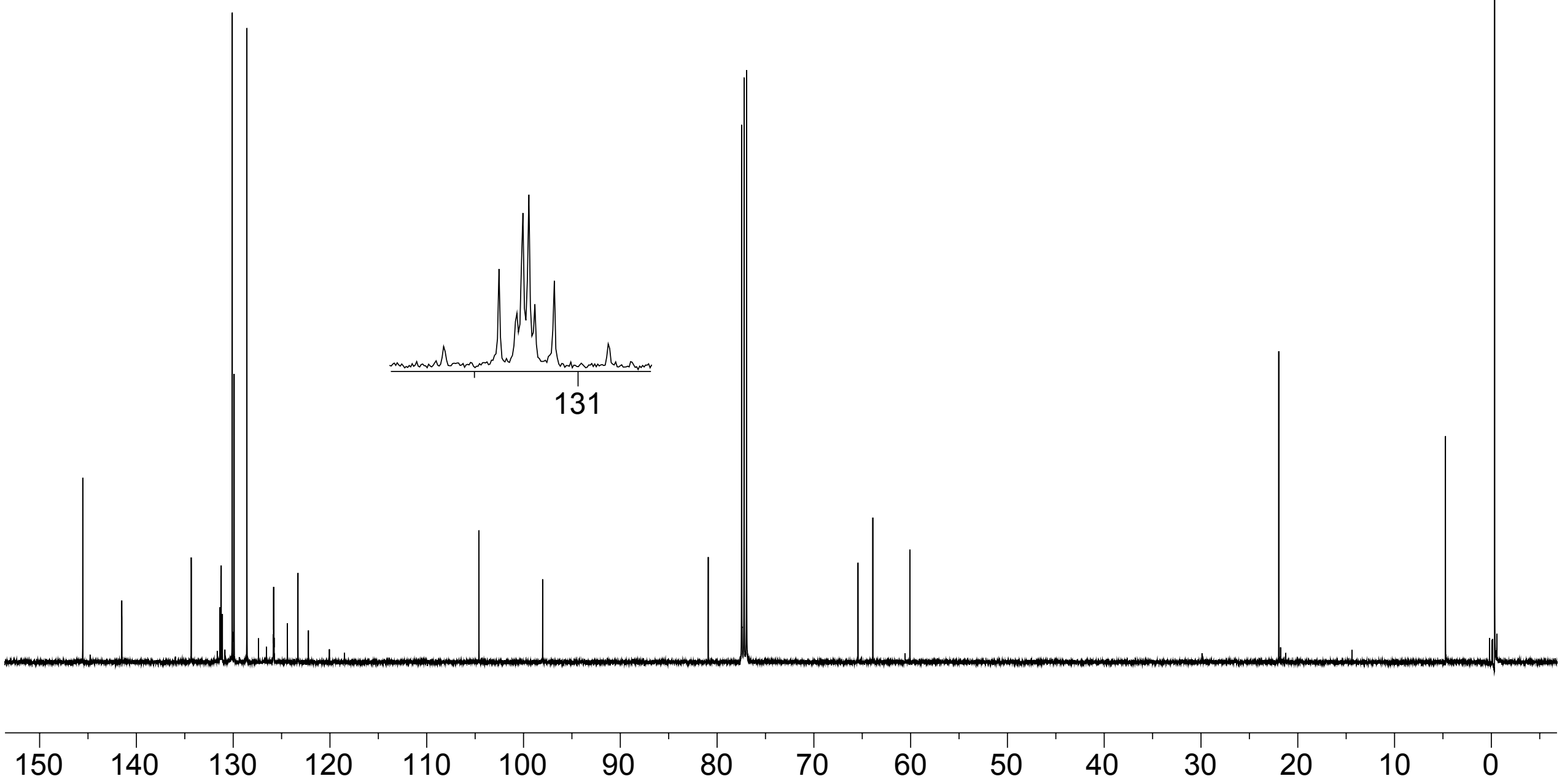




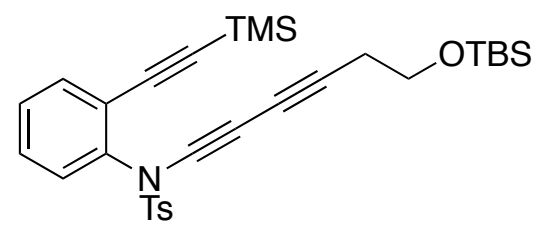

\section{4-TMS/tOTBS}

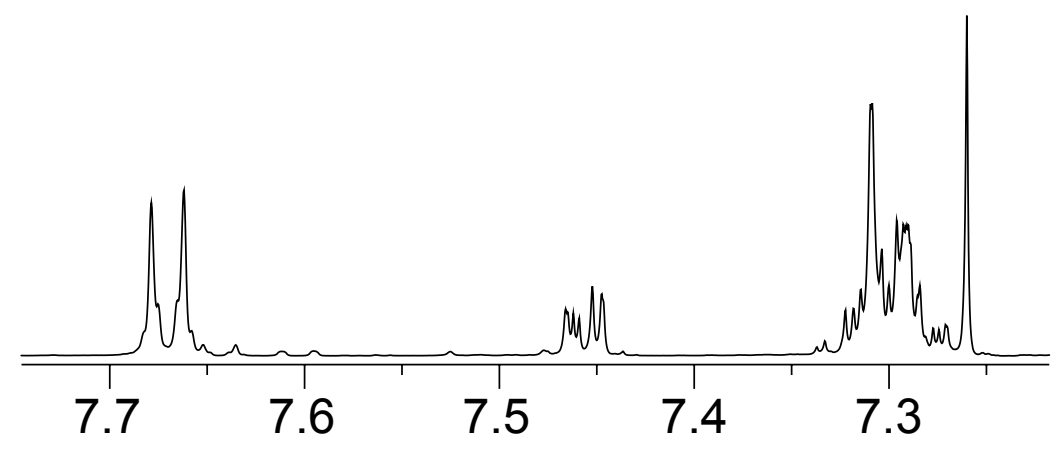




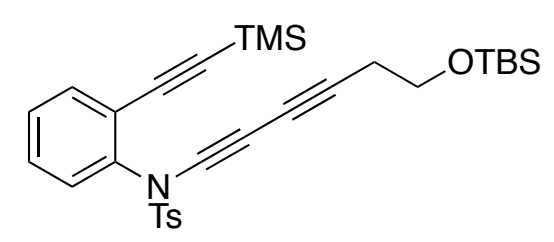

\section{4-TMS/tOTBS}

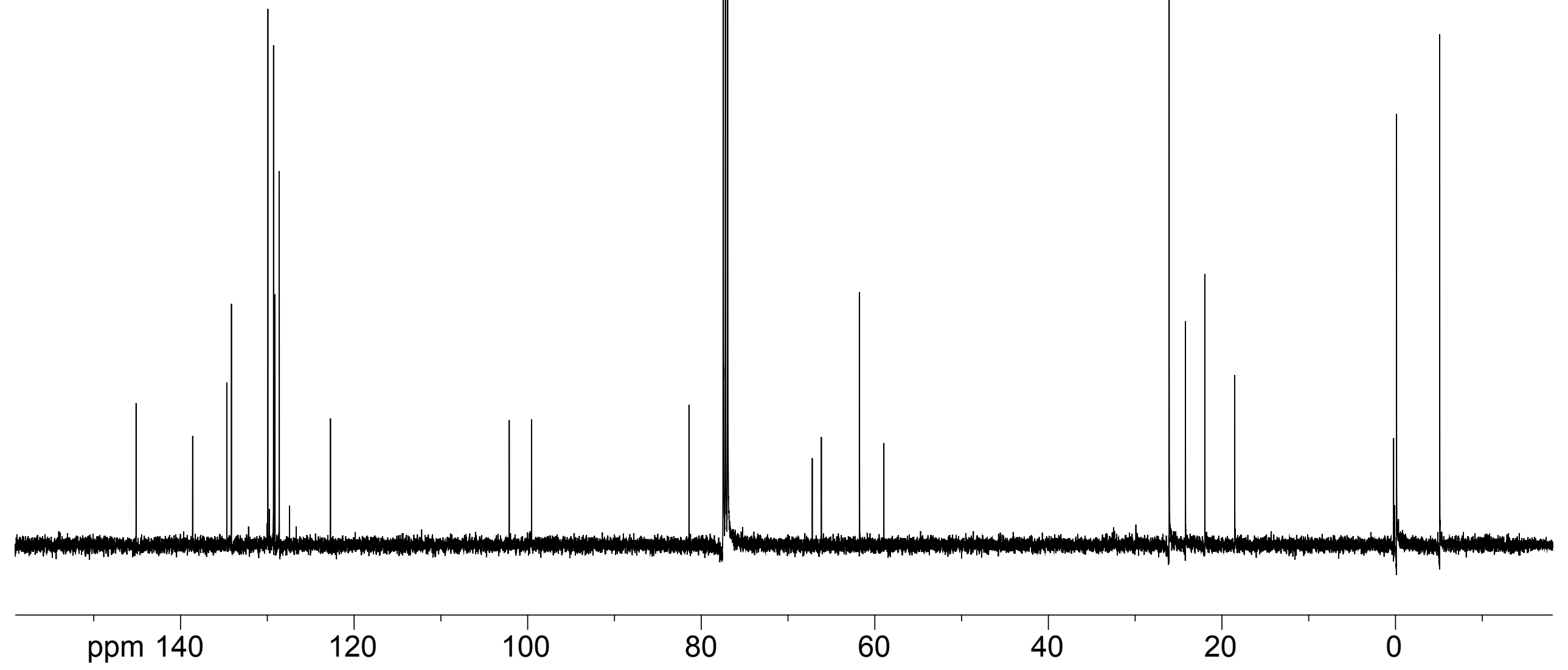



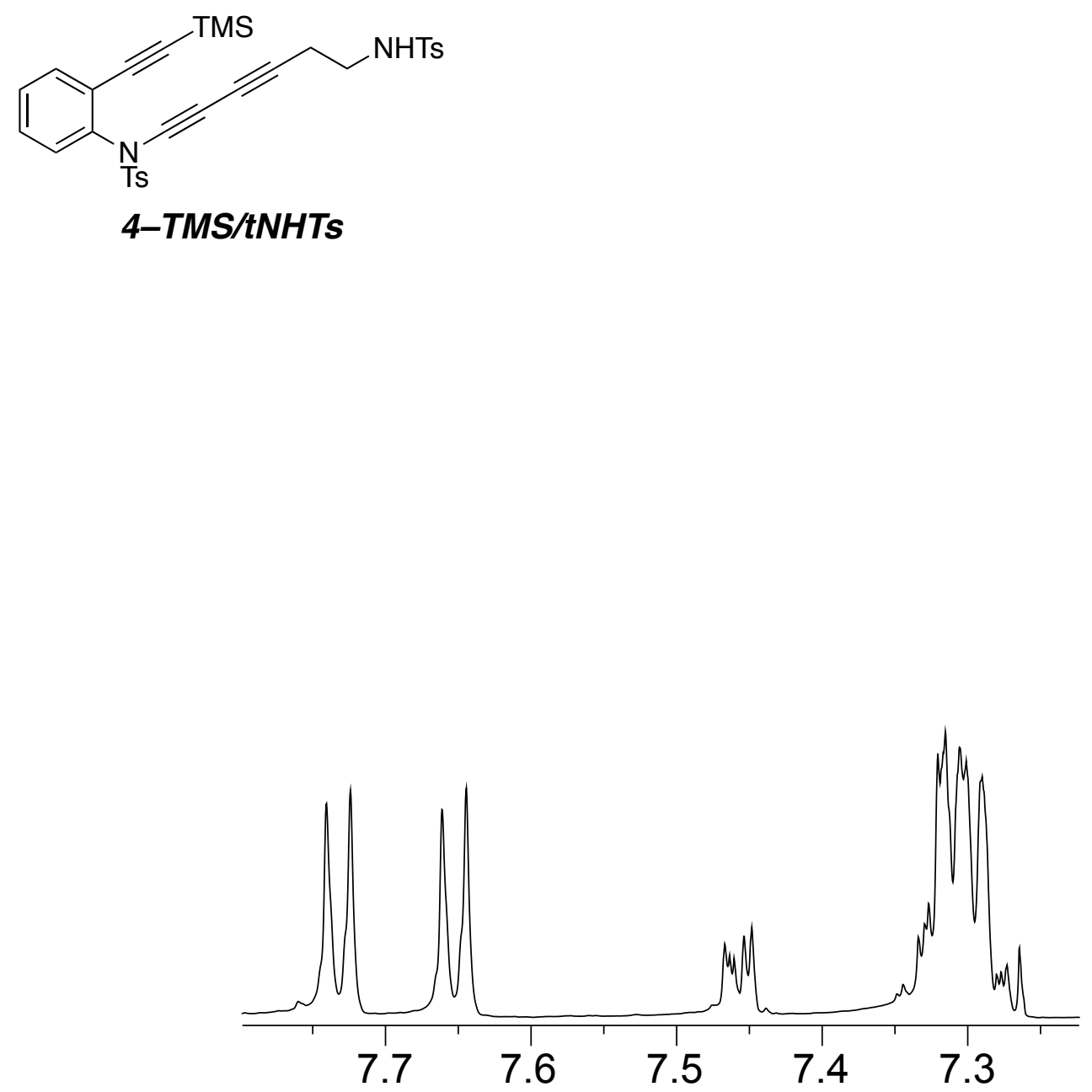

$\|$
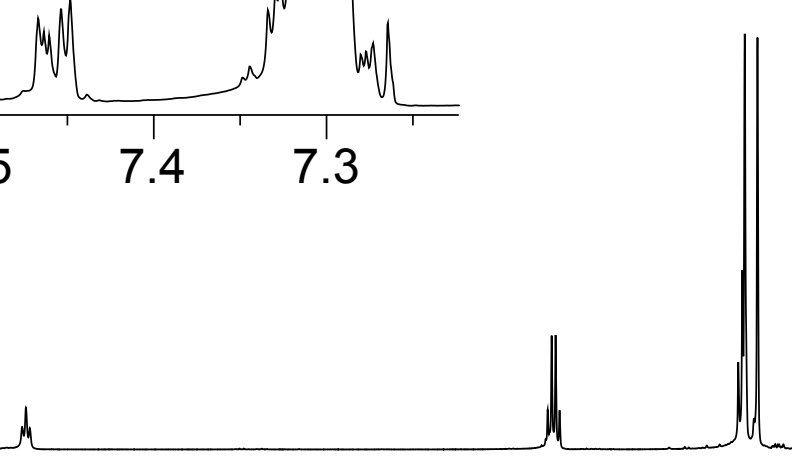

8

7

6

5

4

3

2

1

0 

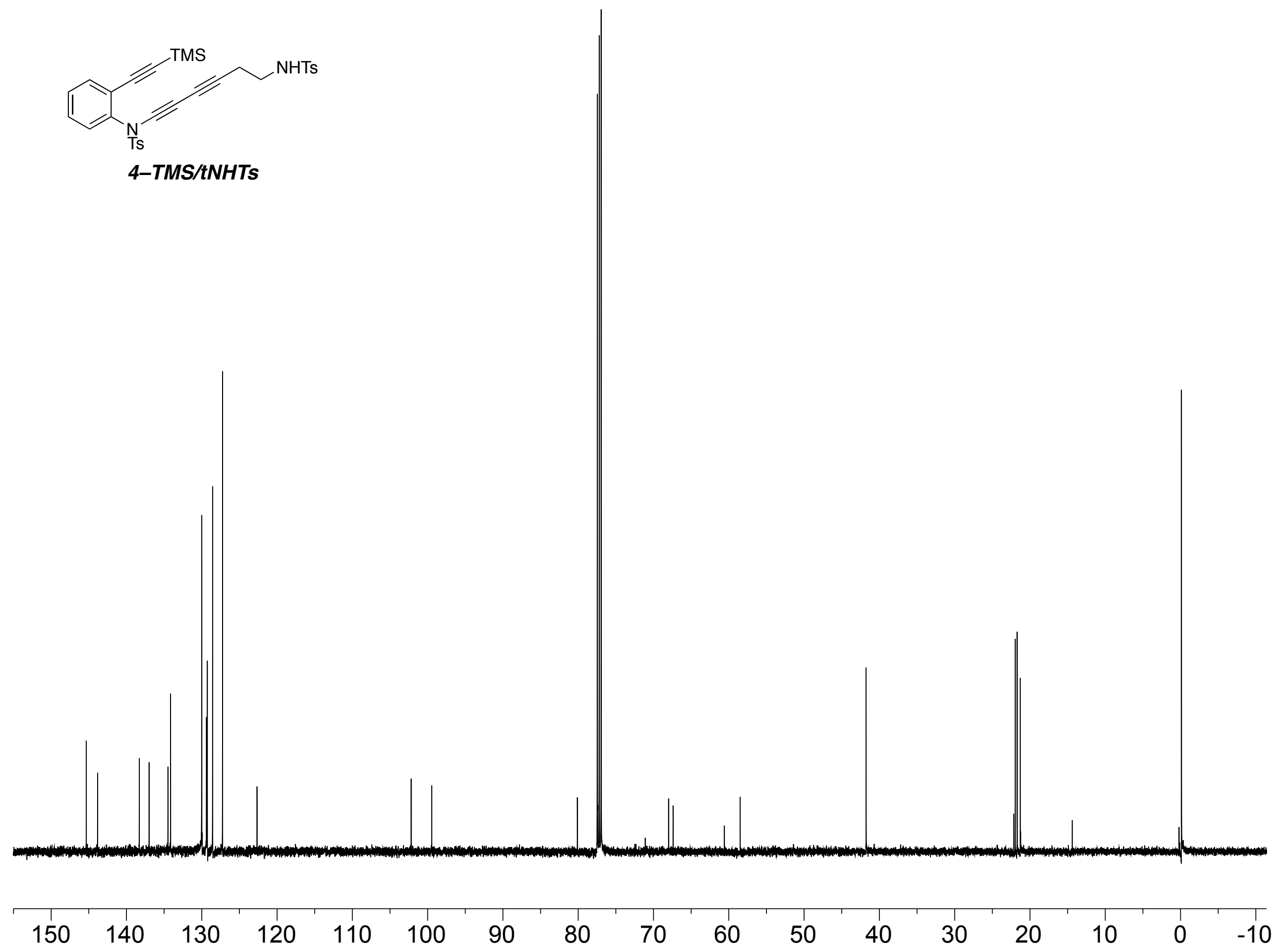

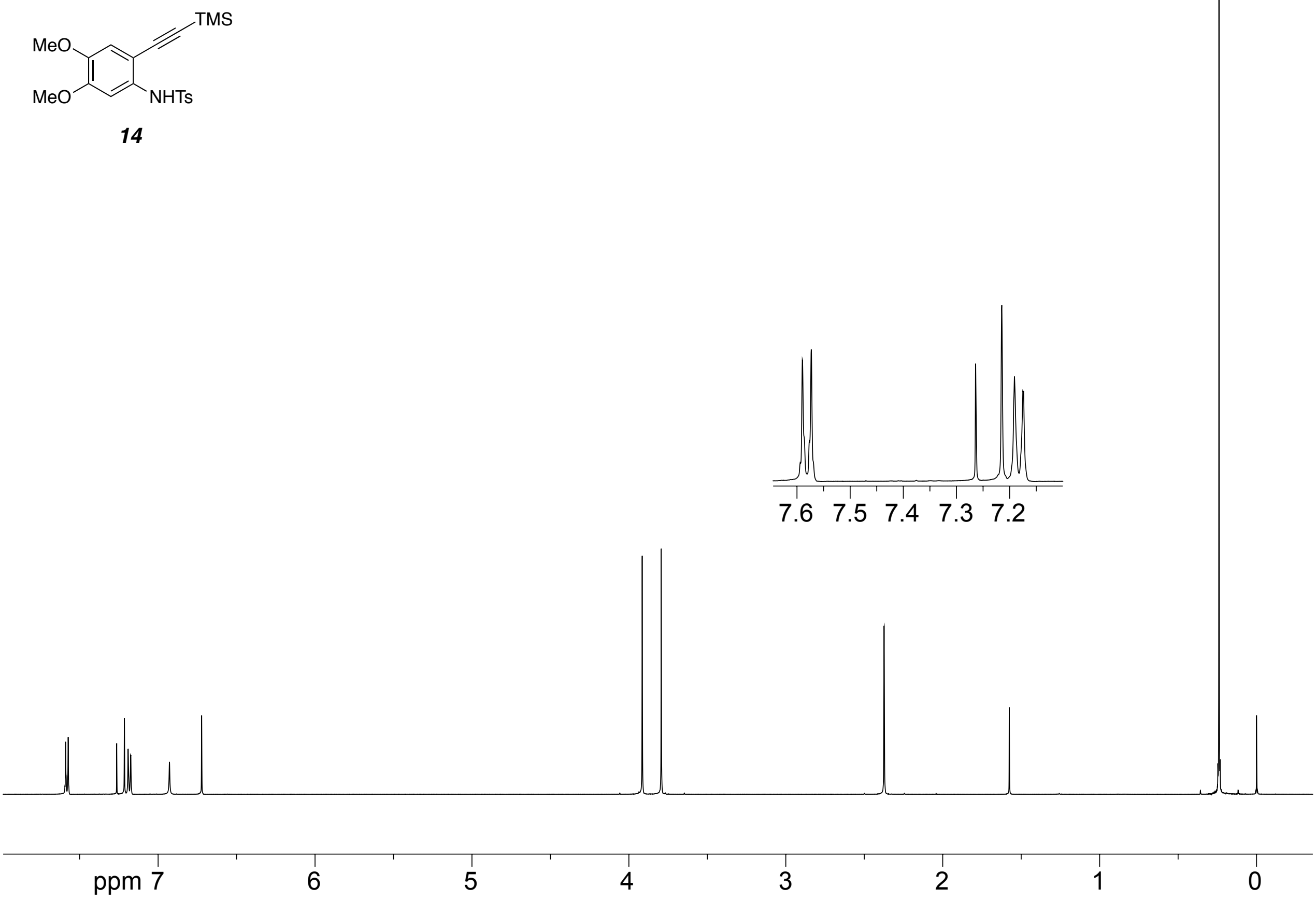

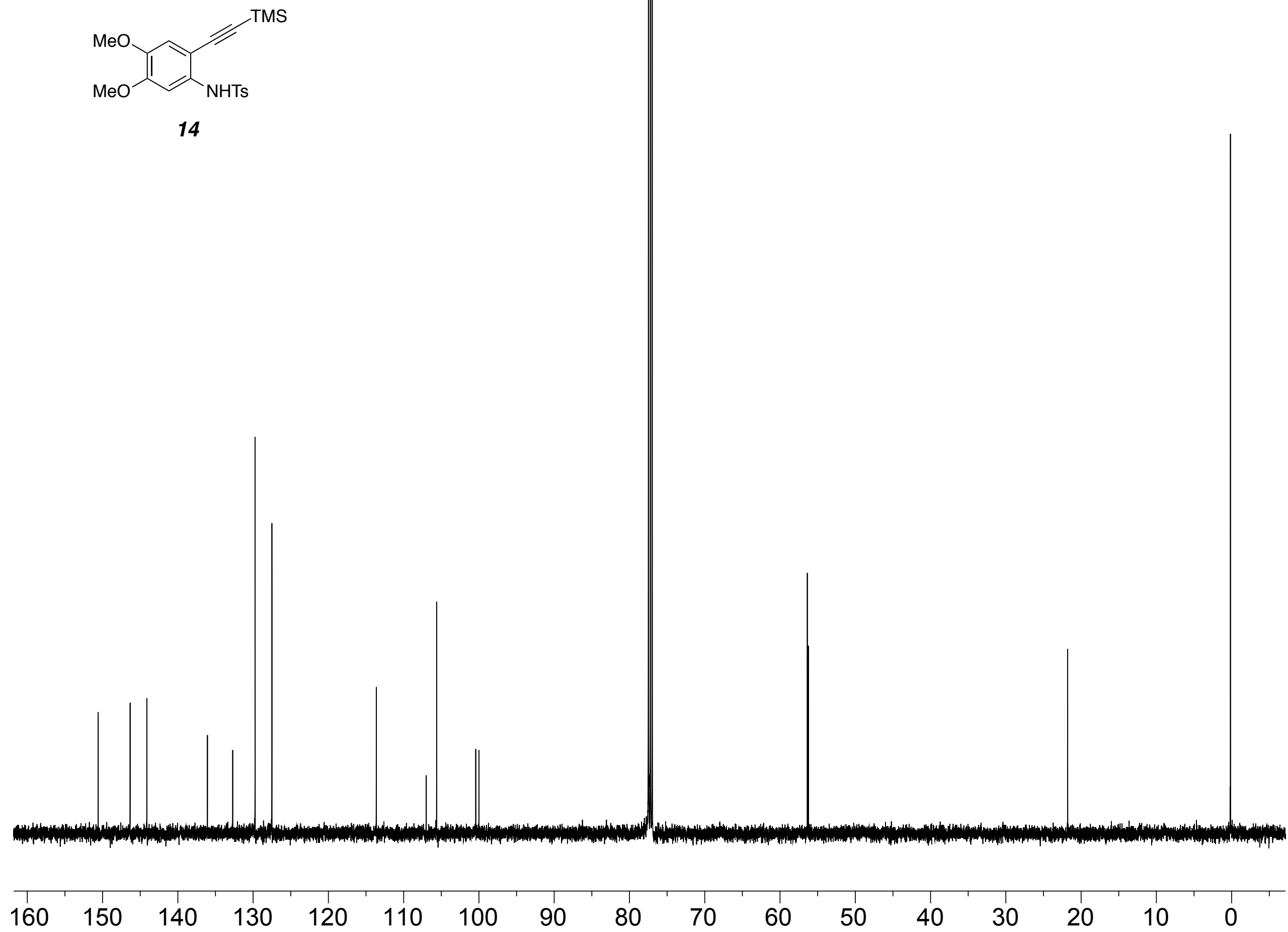


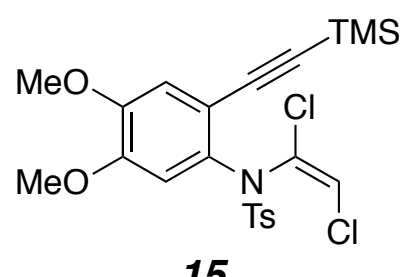

15

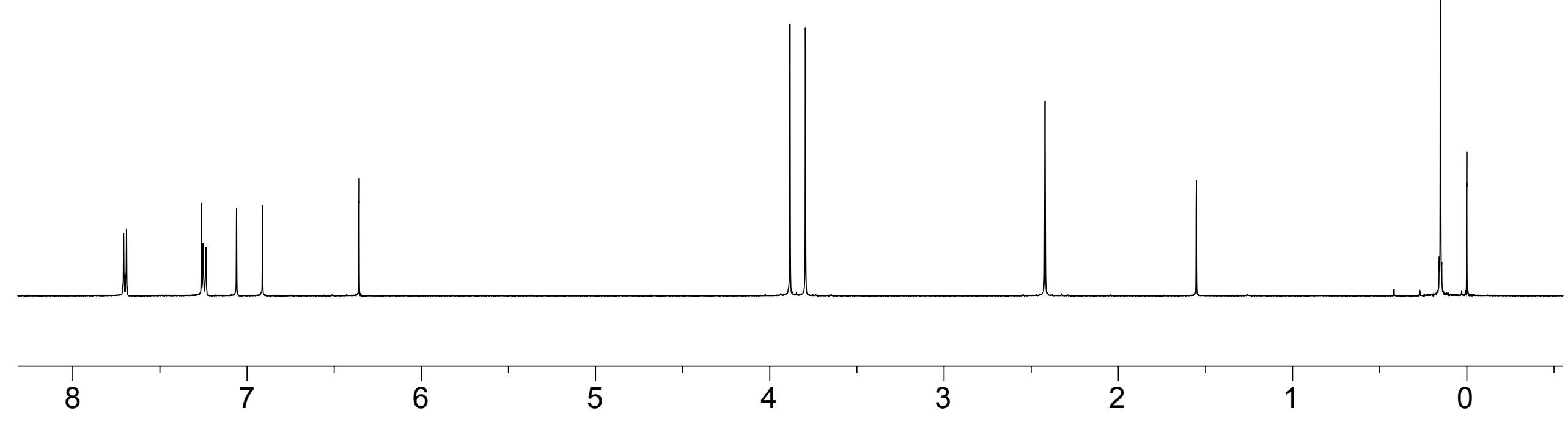




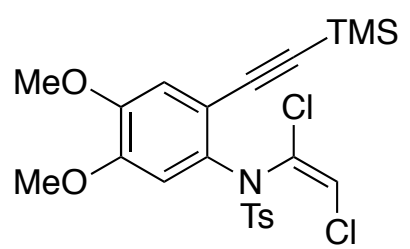

15

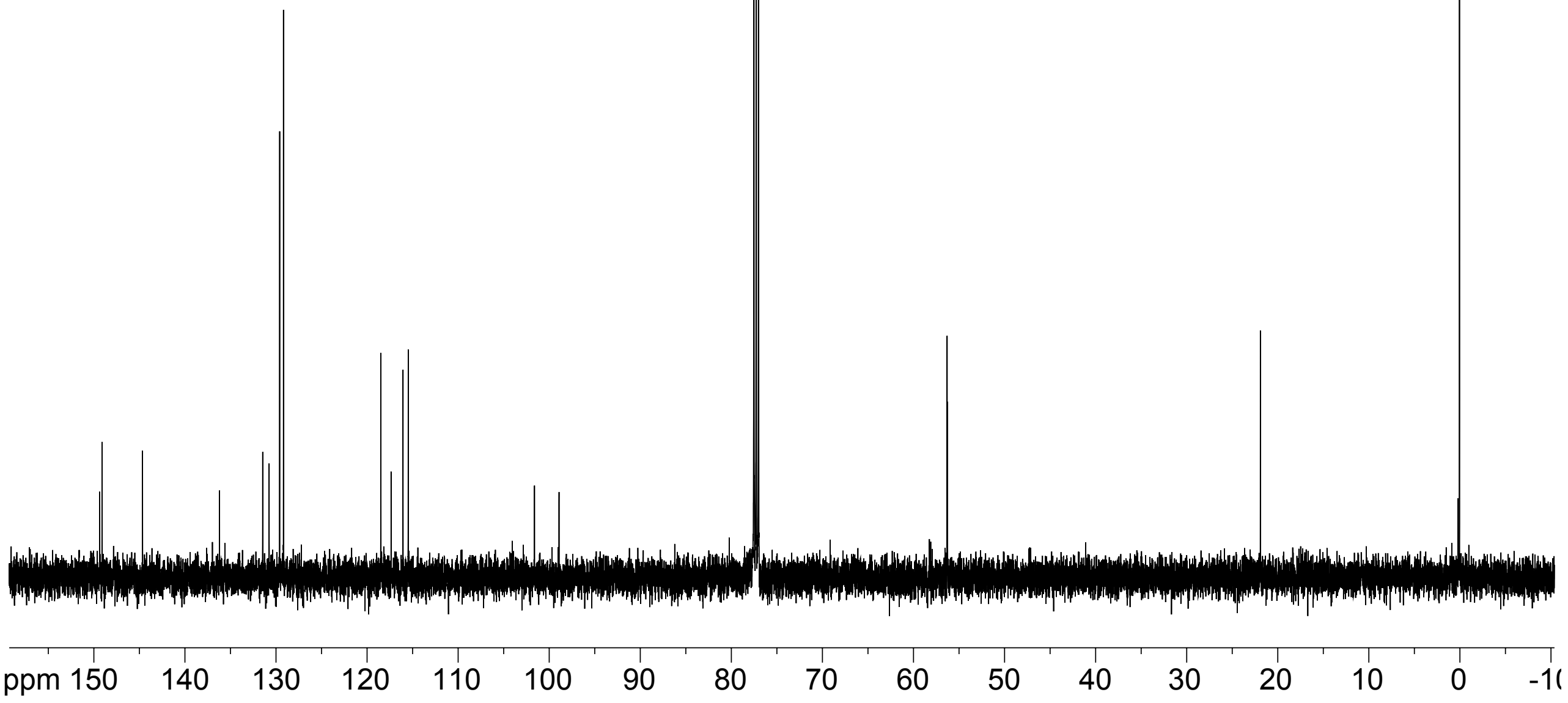



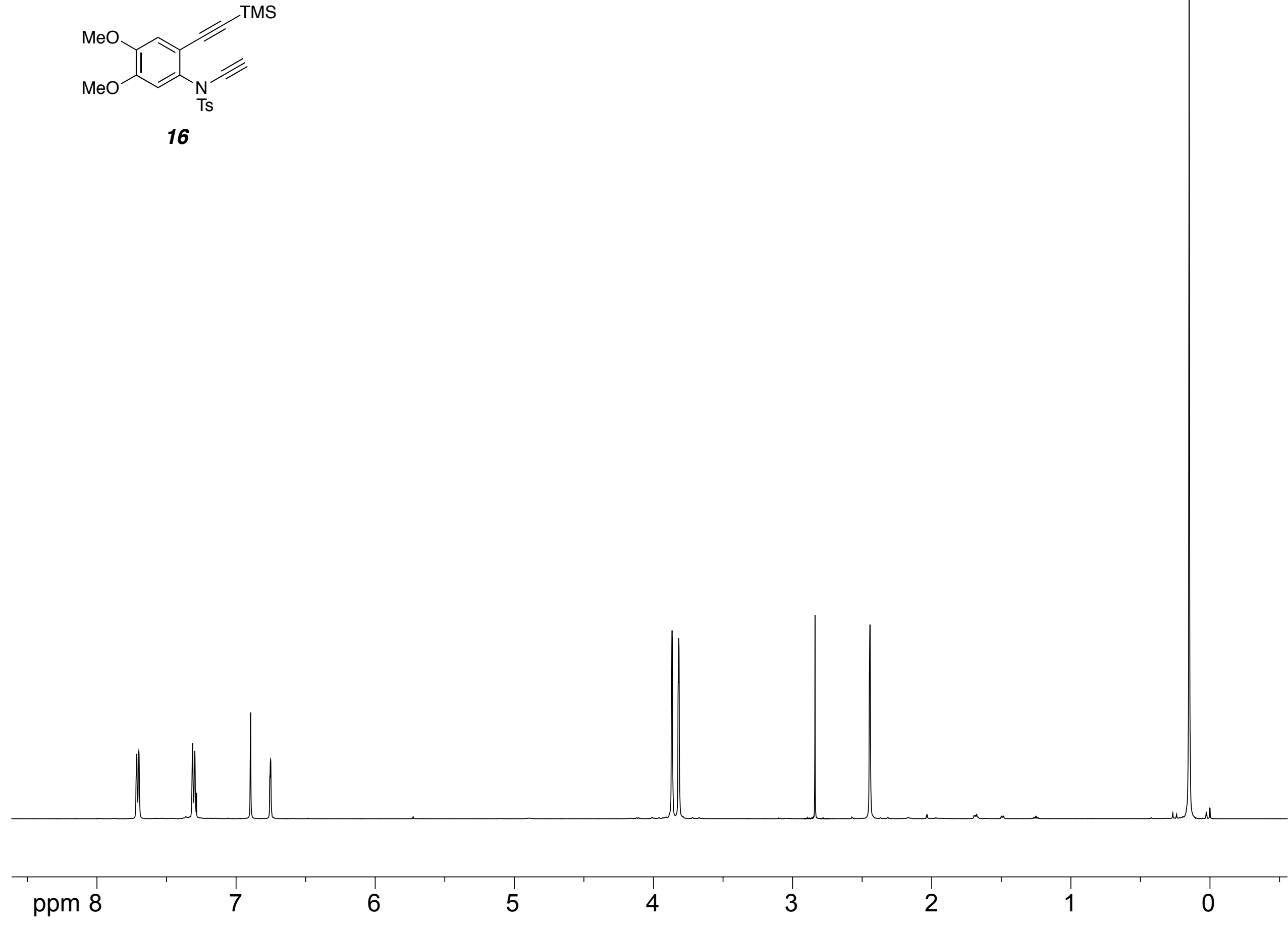

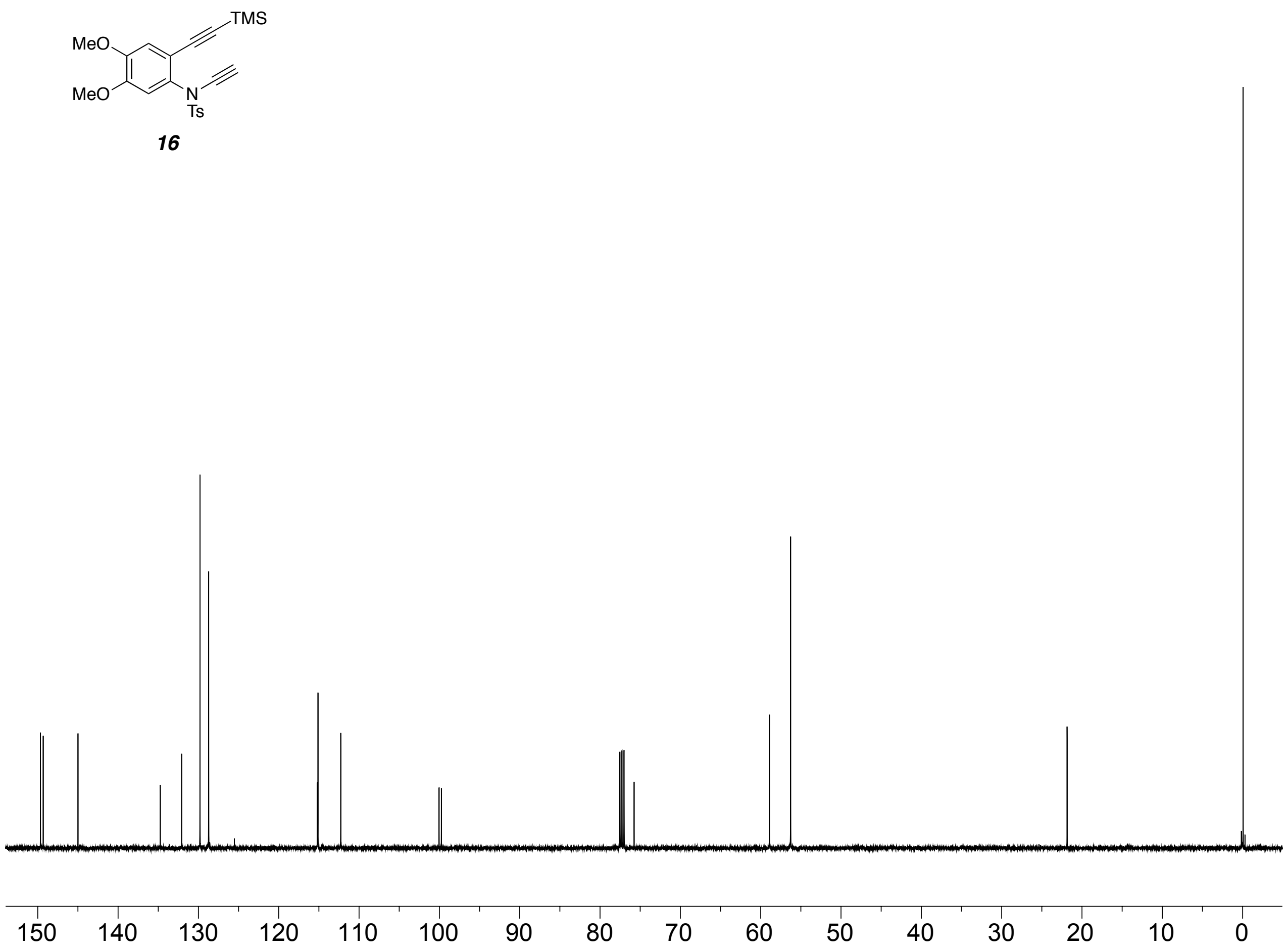

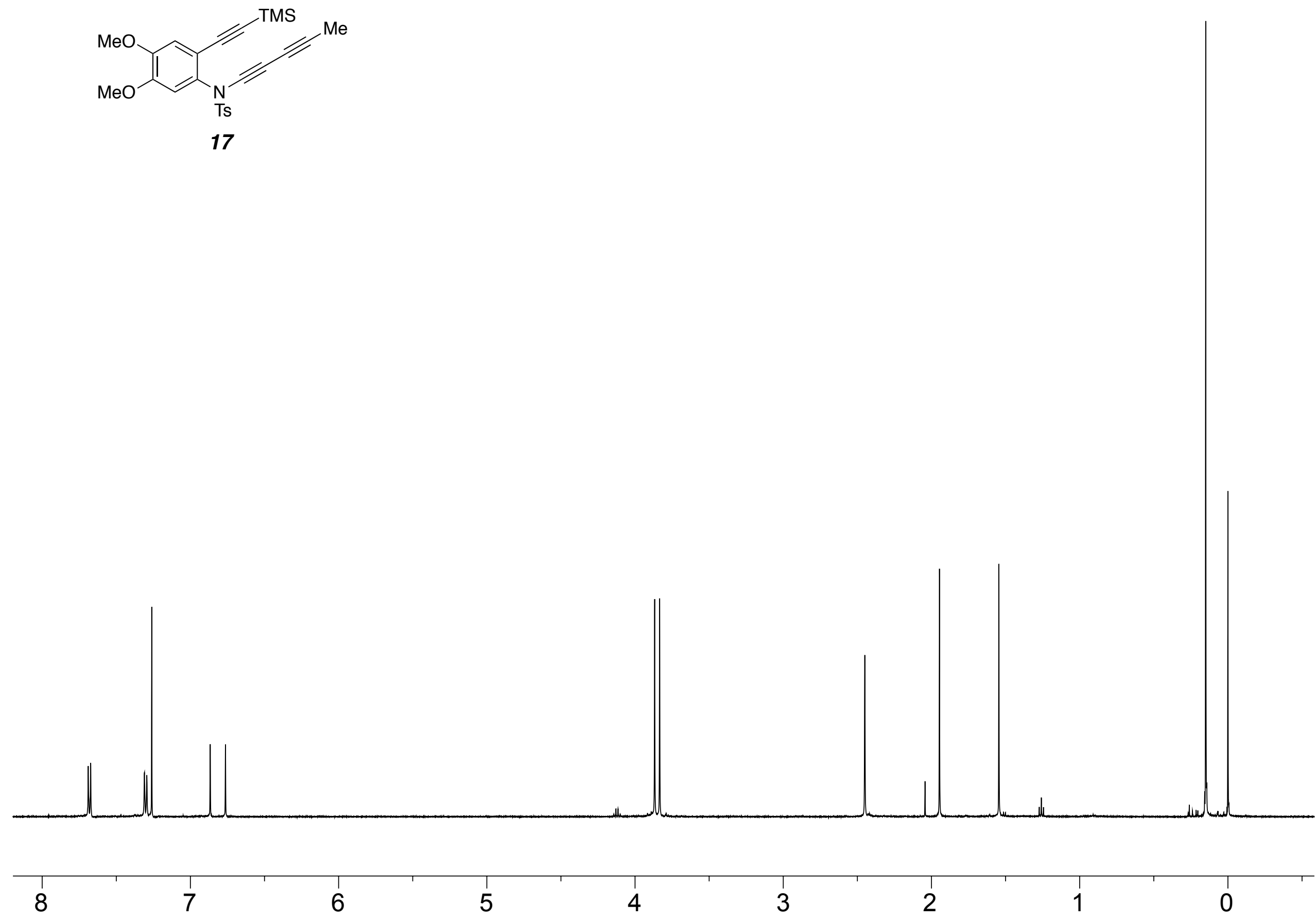

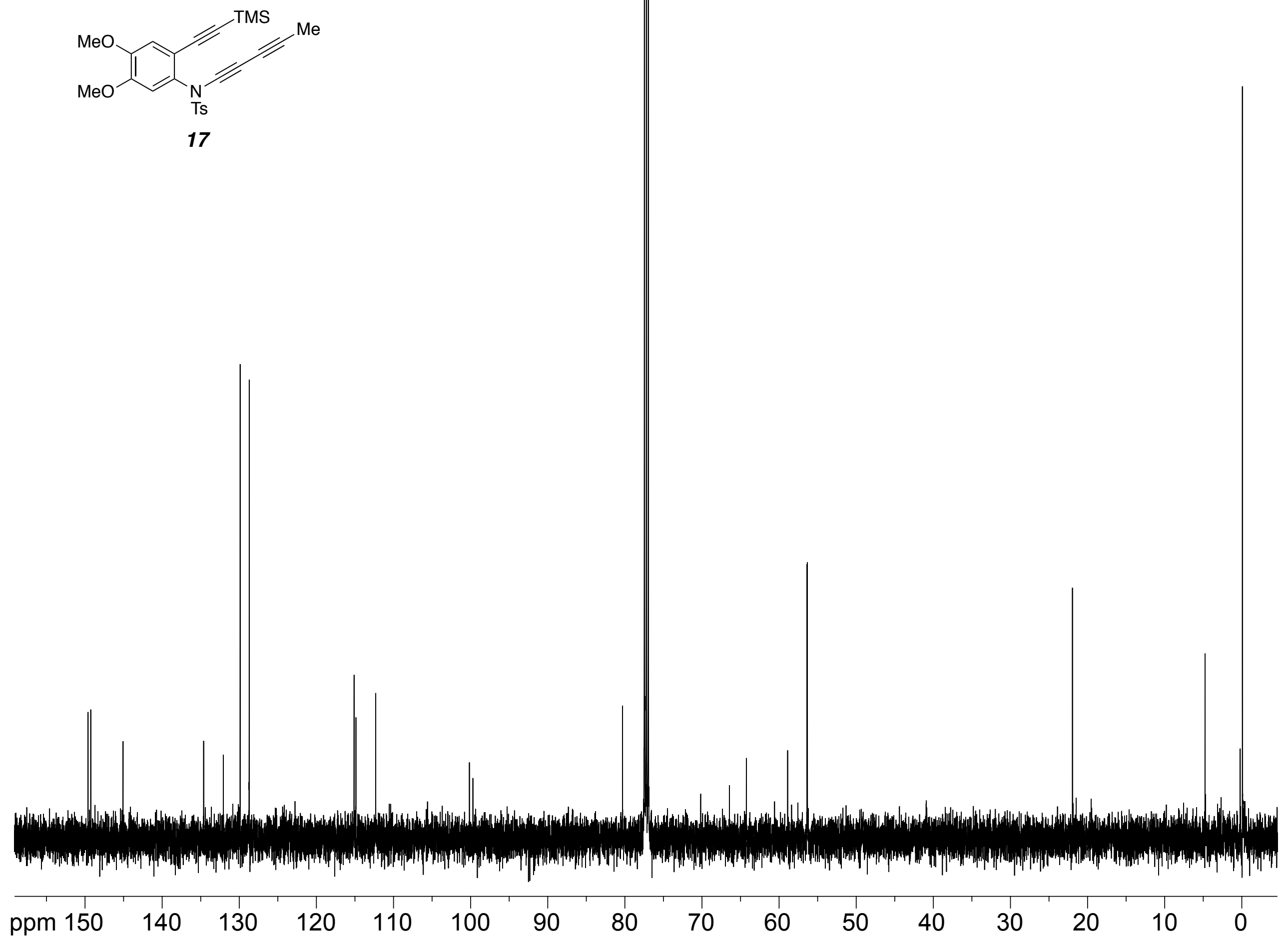

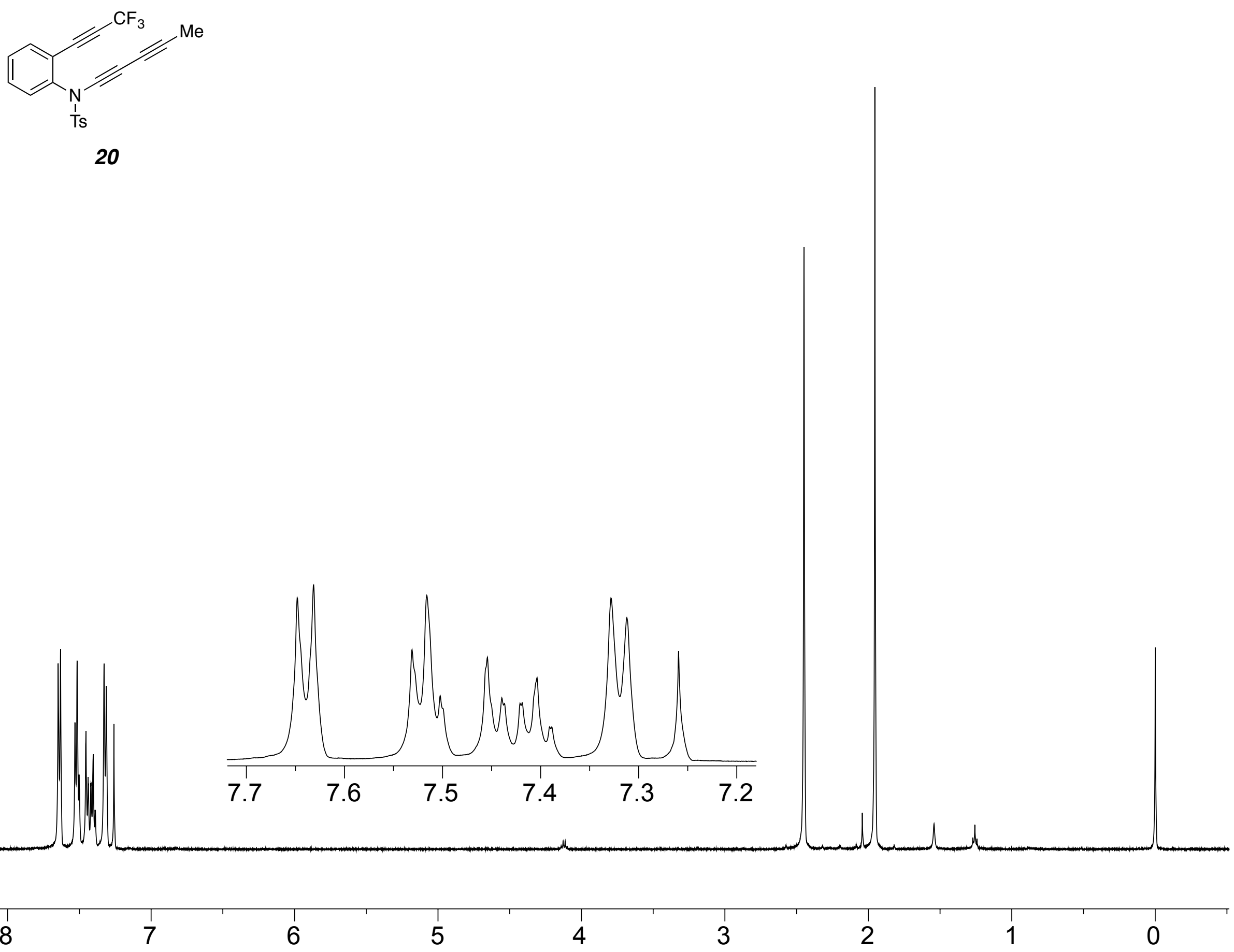

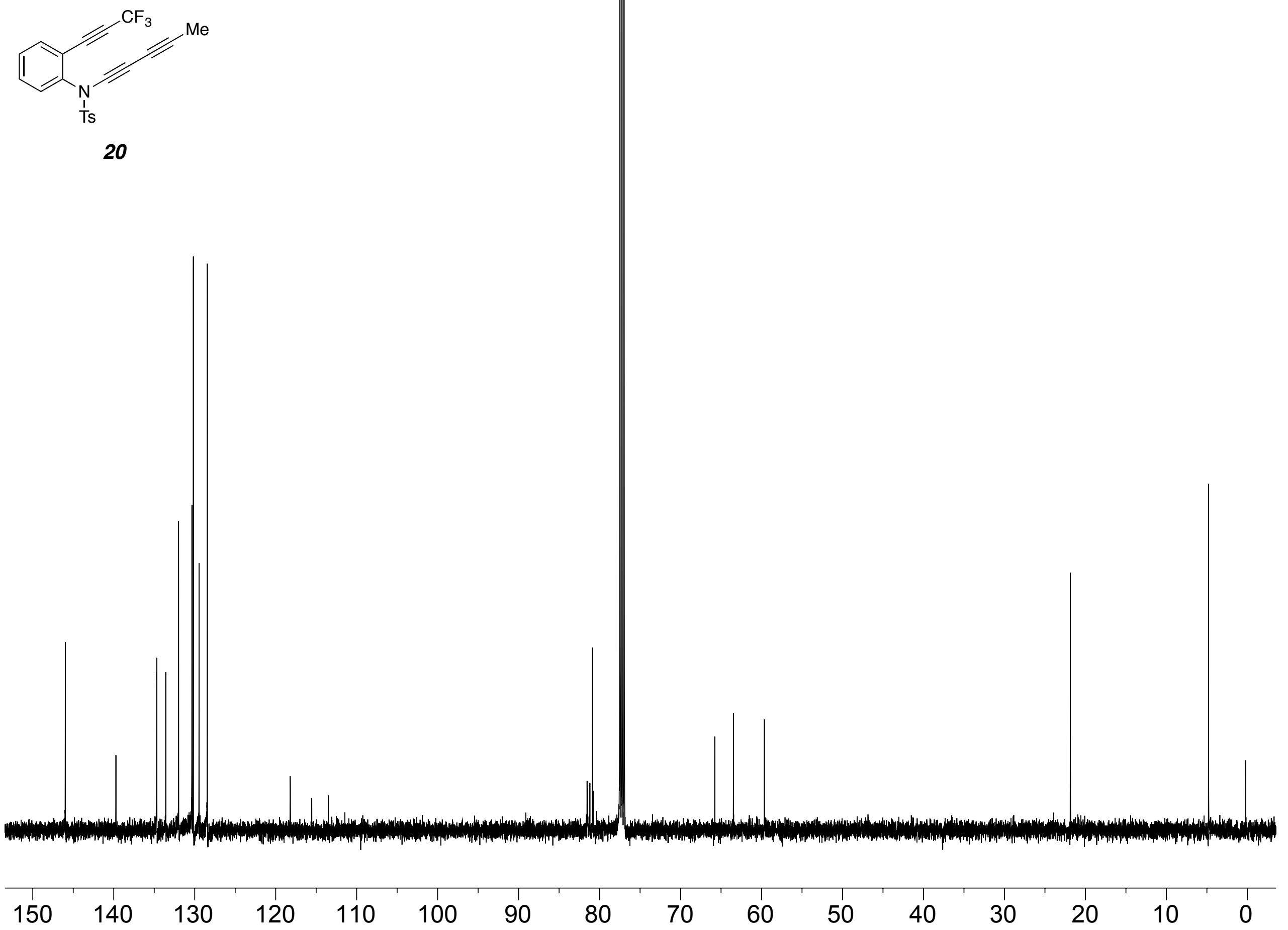

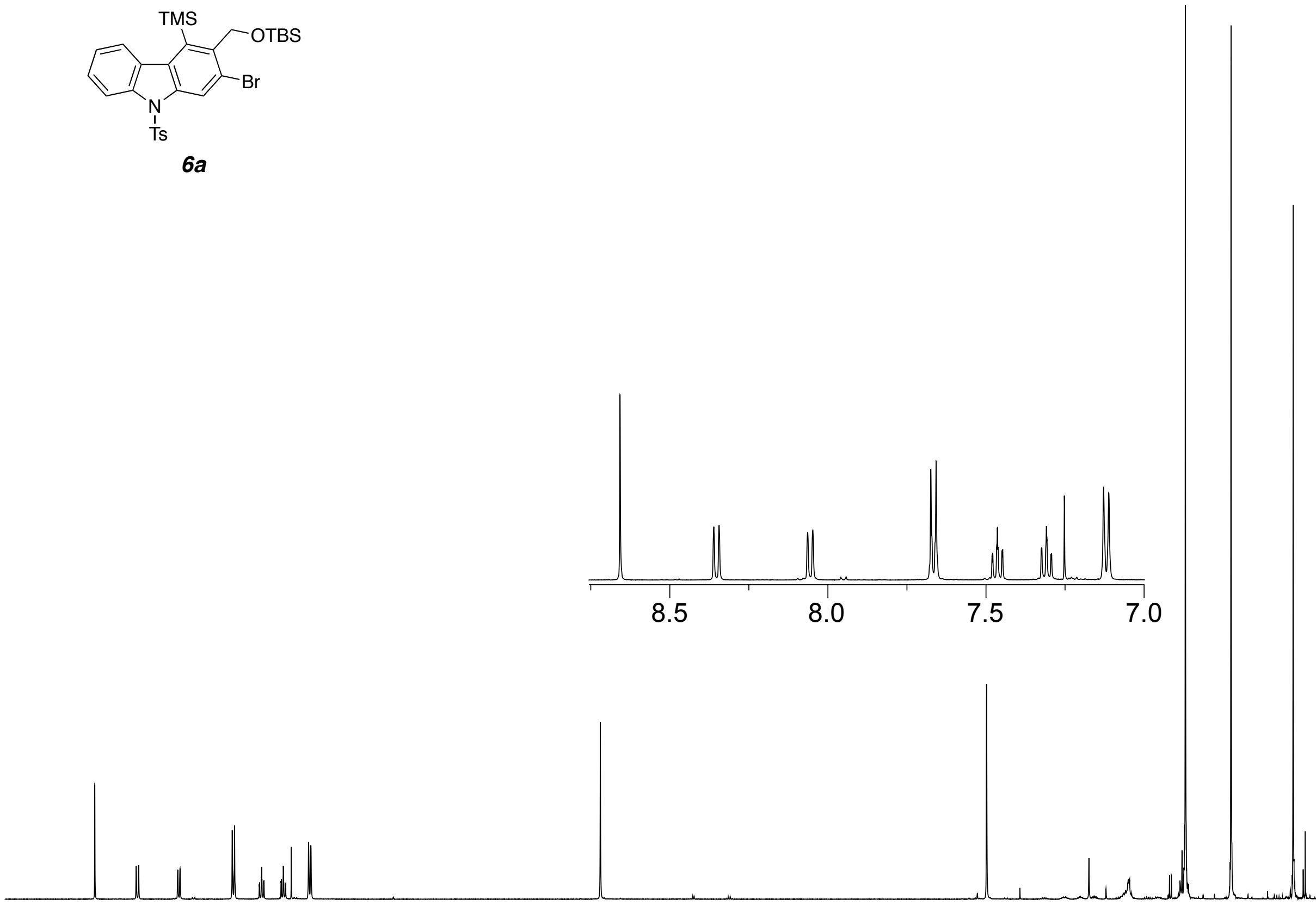

\begin{tabular}{ll|l|l|l|l|l|l|l|l|l|l|}
\hline 9 & 8 & 7 & 6 & 5 & 4 & 3 & 2 & 1 & 0
\end{tabular}



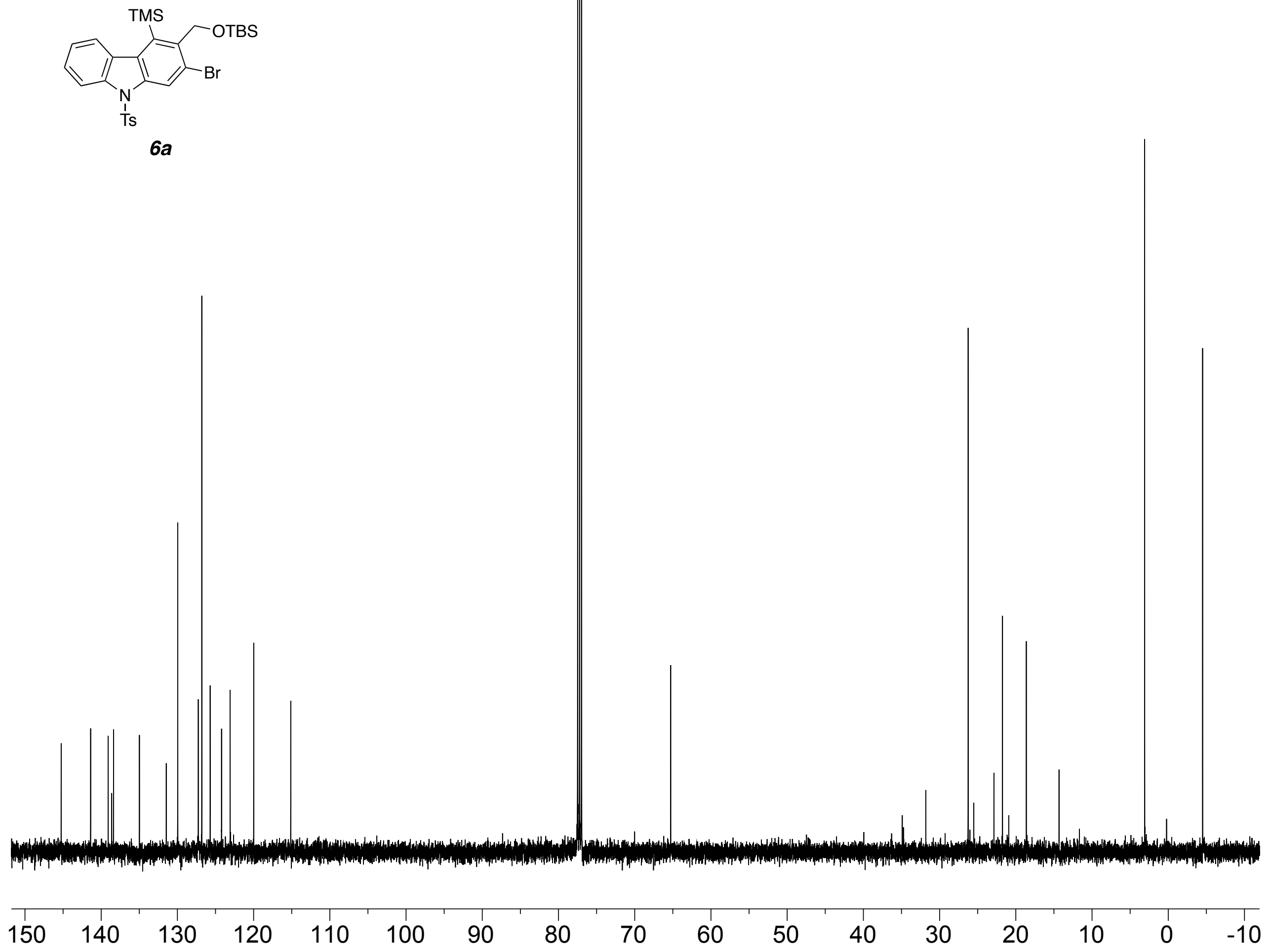


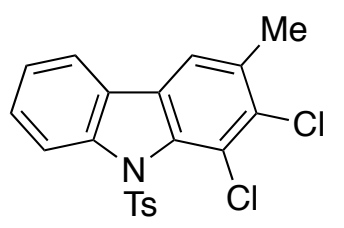

$6 b$

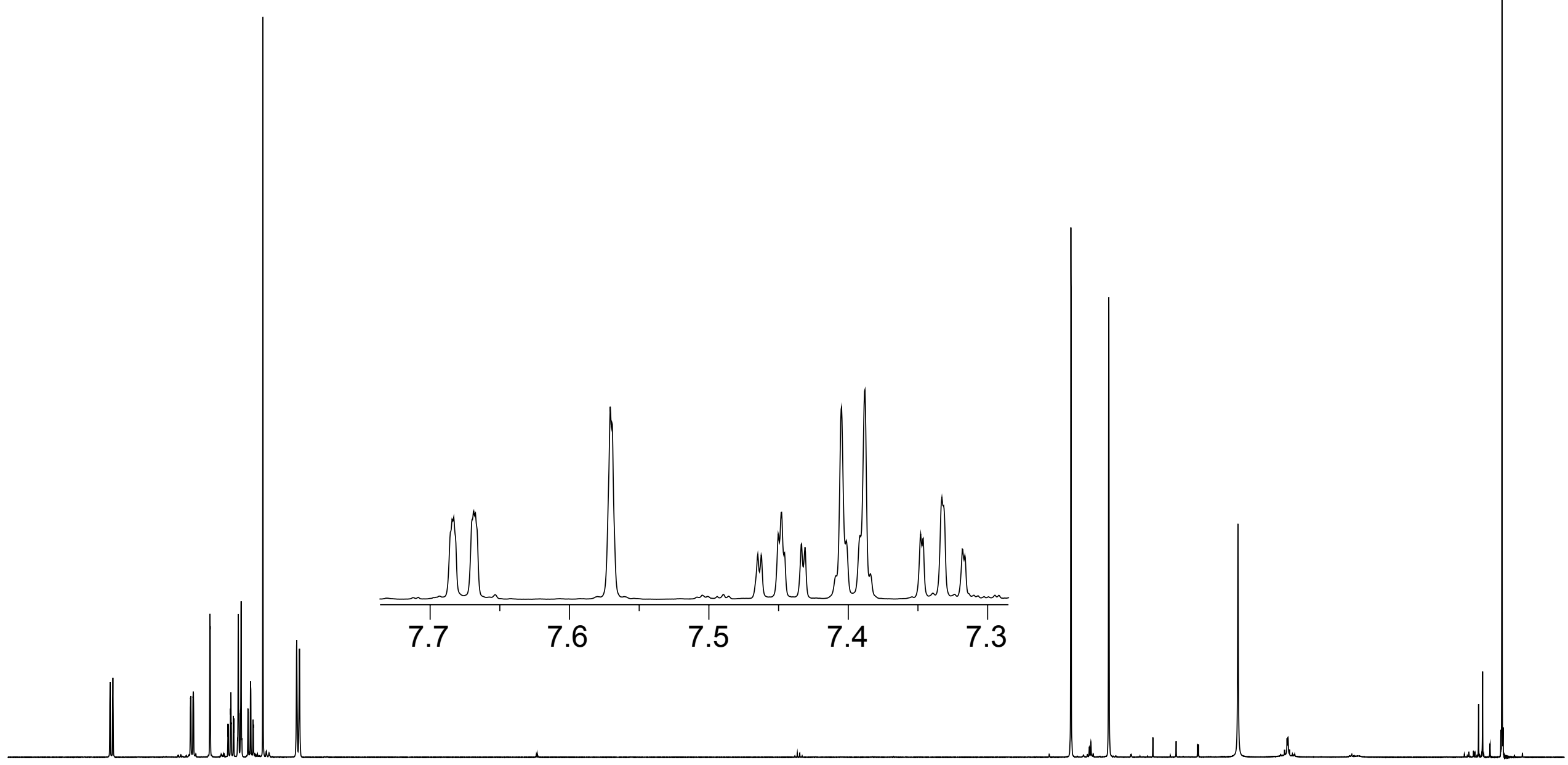

ppm 8

7

6

5

4

3

2

1

0 
Supporting Information

qarbazole

Page S58 of S82

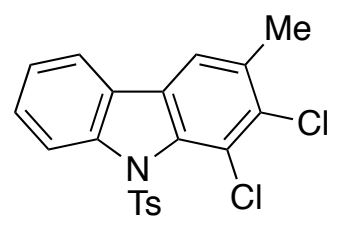

$6 b$

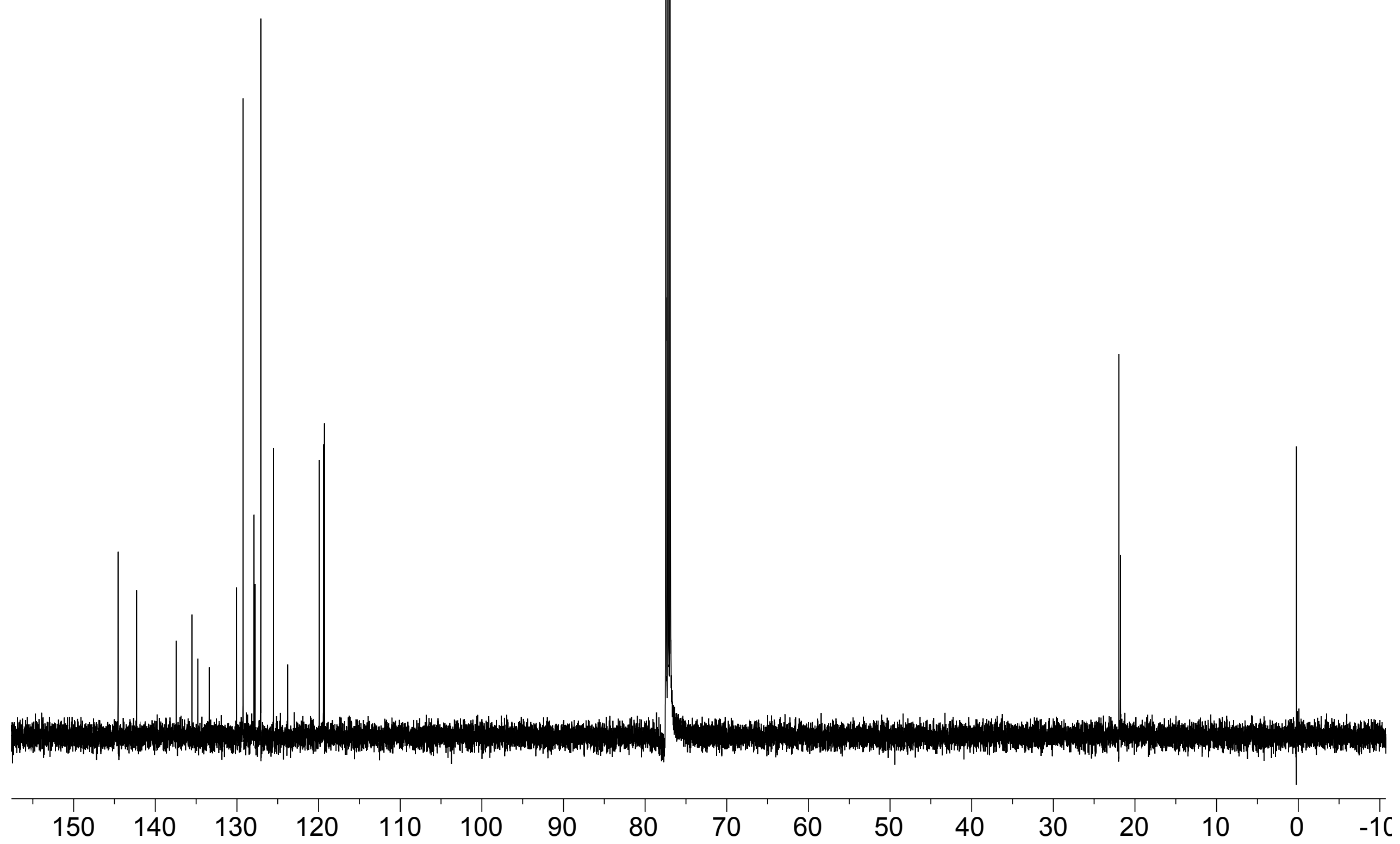




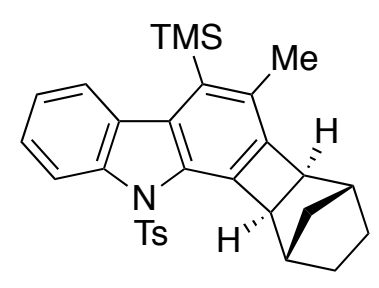

$6 c$

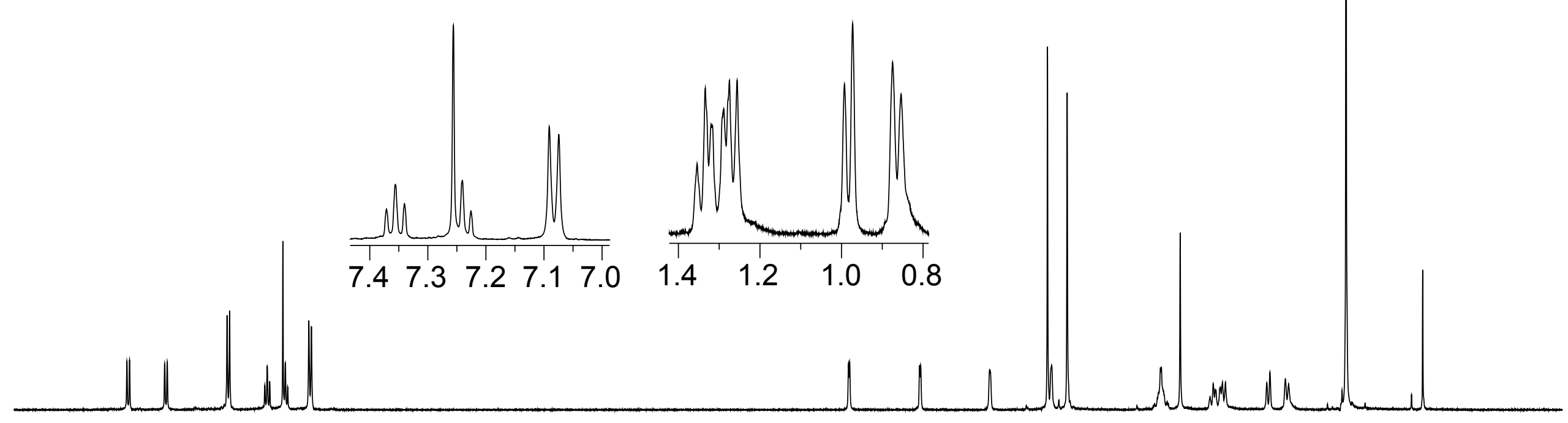




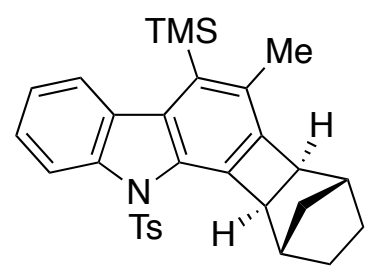

$6 c$
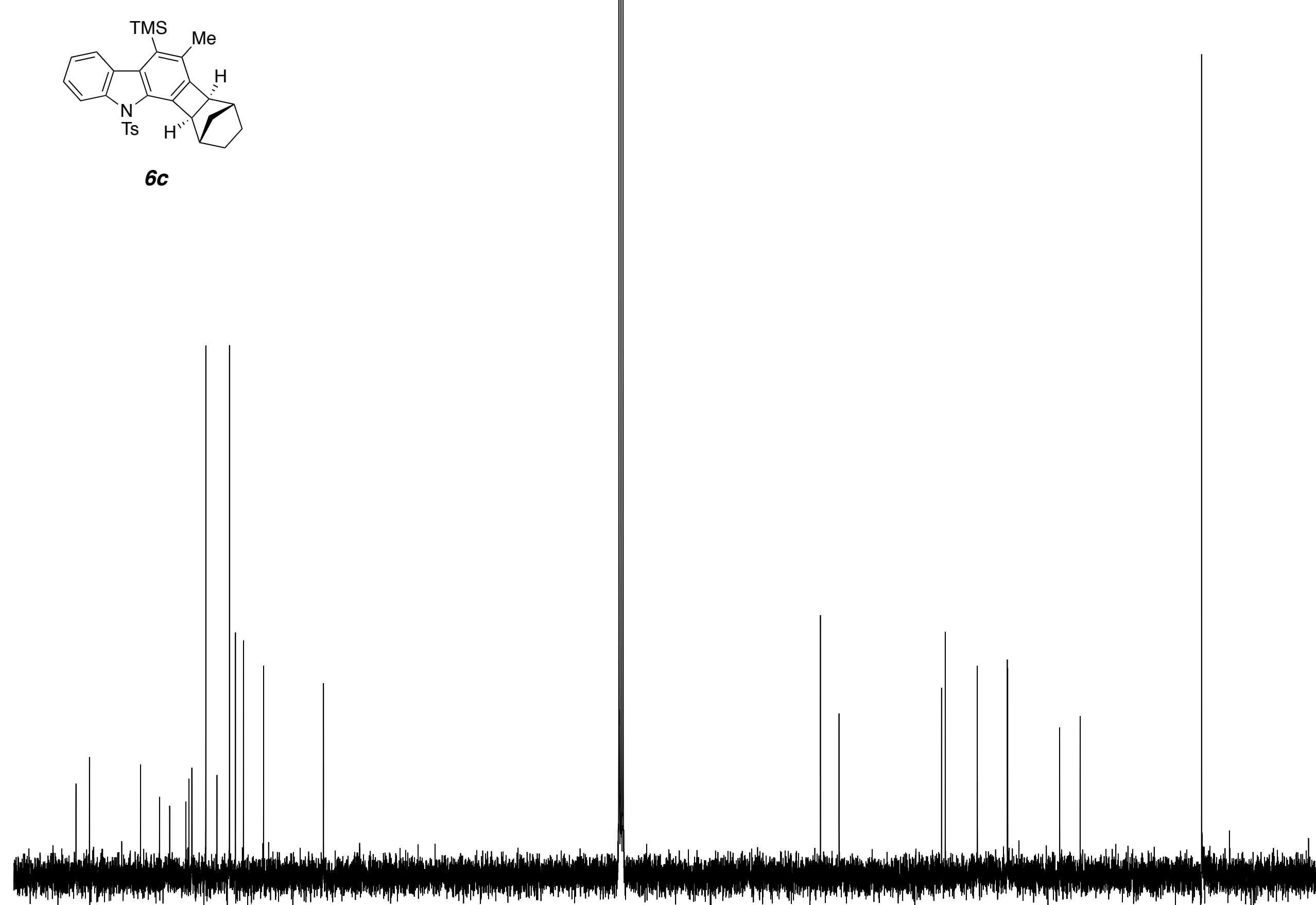


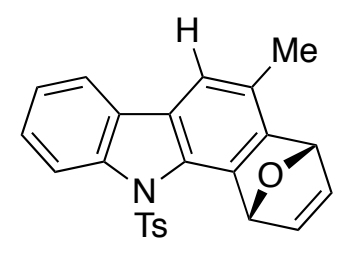

$6 d$

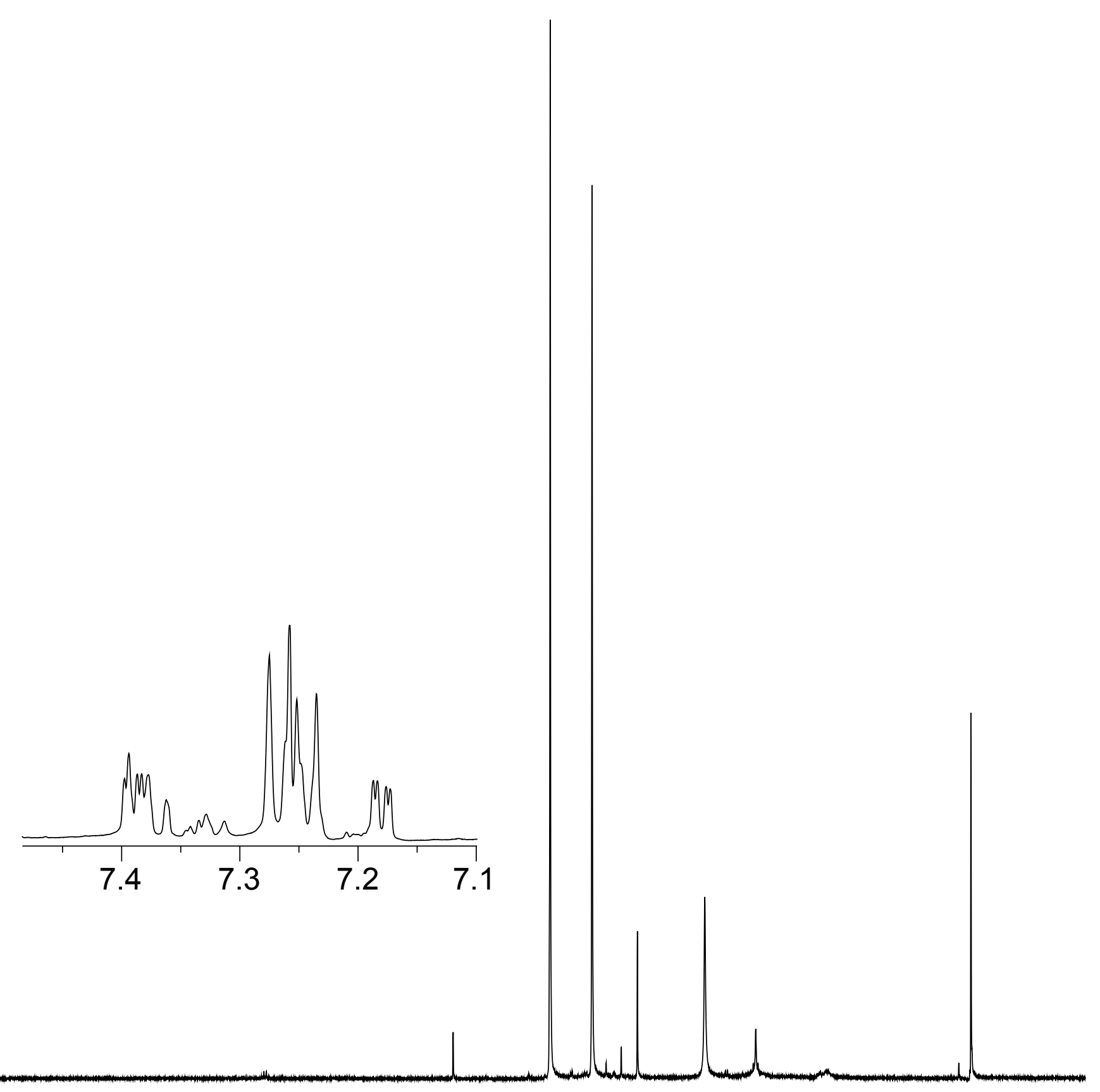

ppm 8

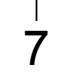

6

5

4

3

2

1 


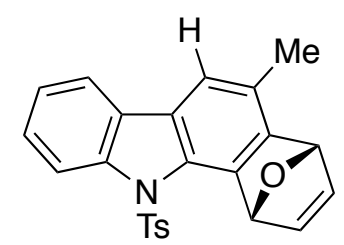

$6 d$

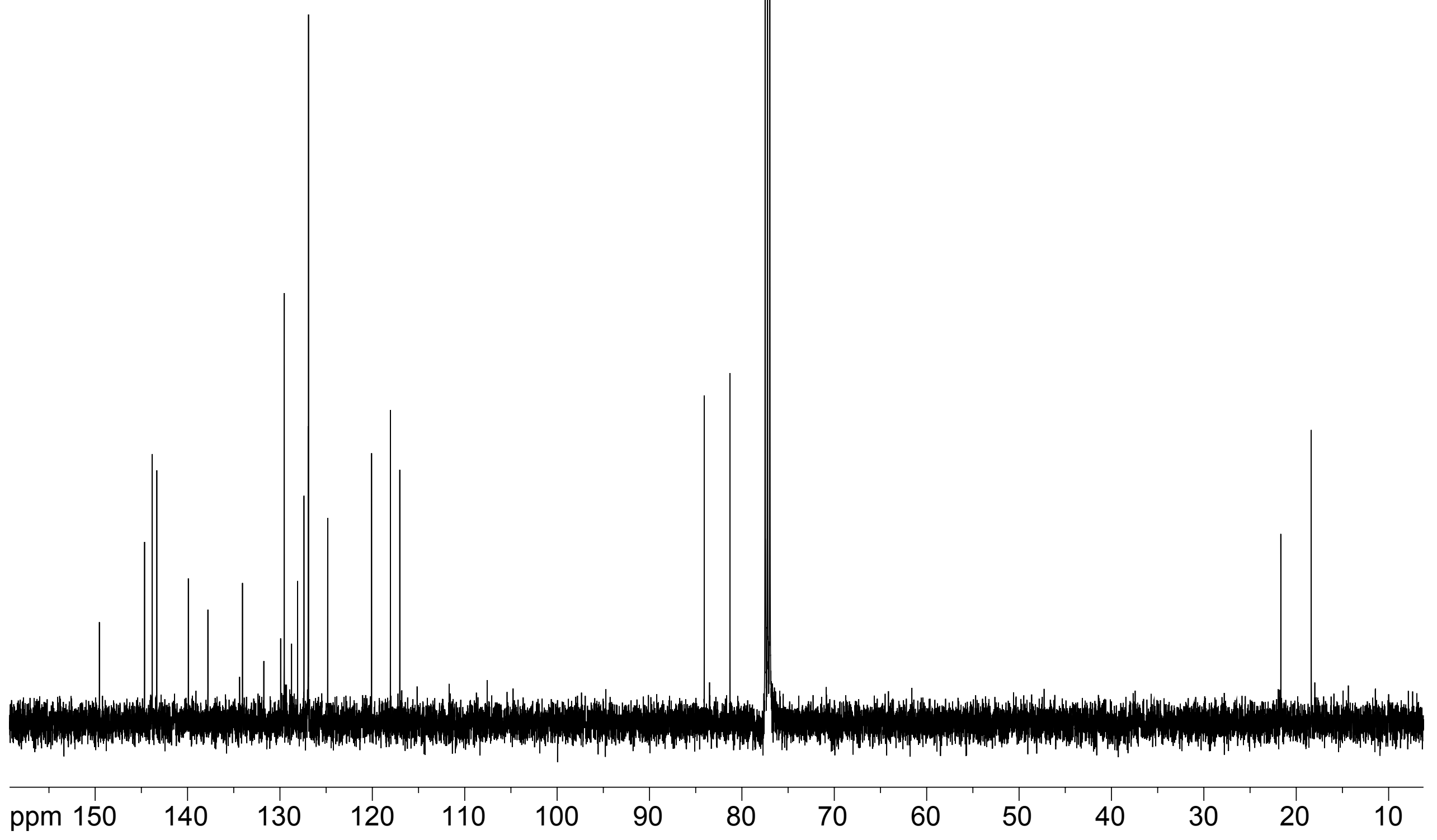




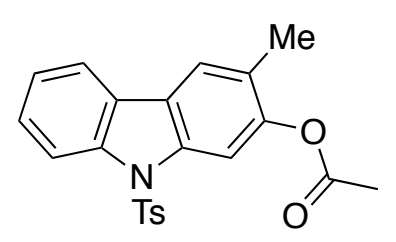

$6 e$

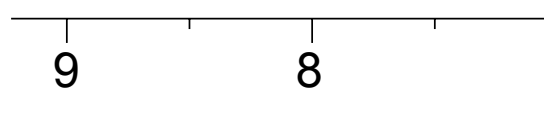

76

$6 \quad 5$

1
5

$4 \quad 3$

32

21

1 


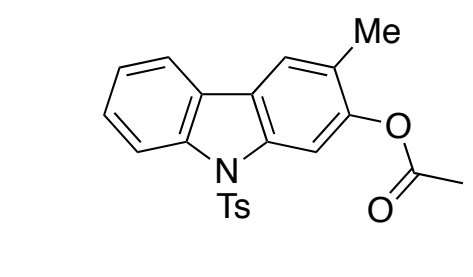

$6 e$

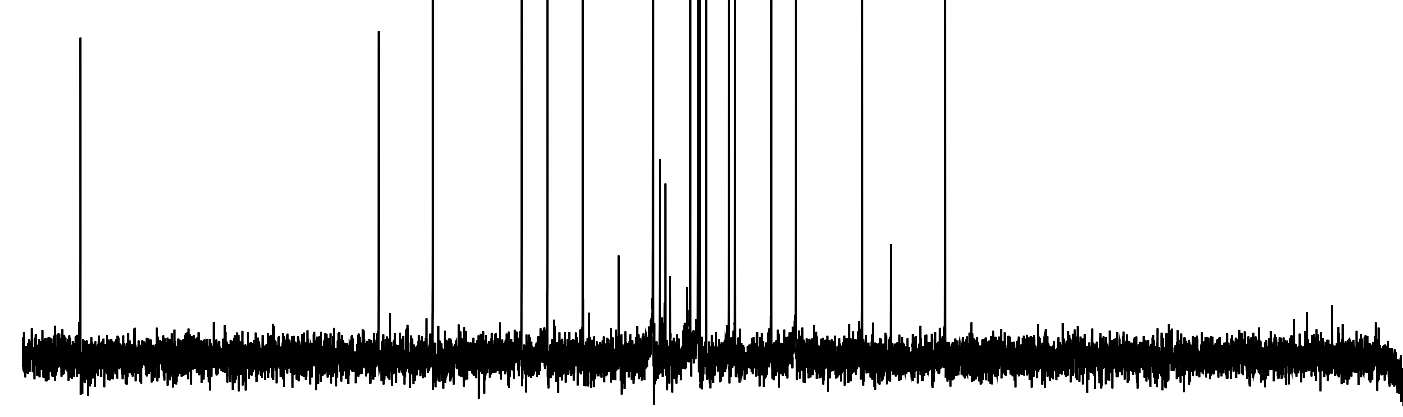



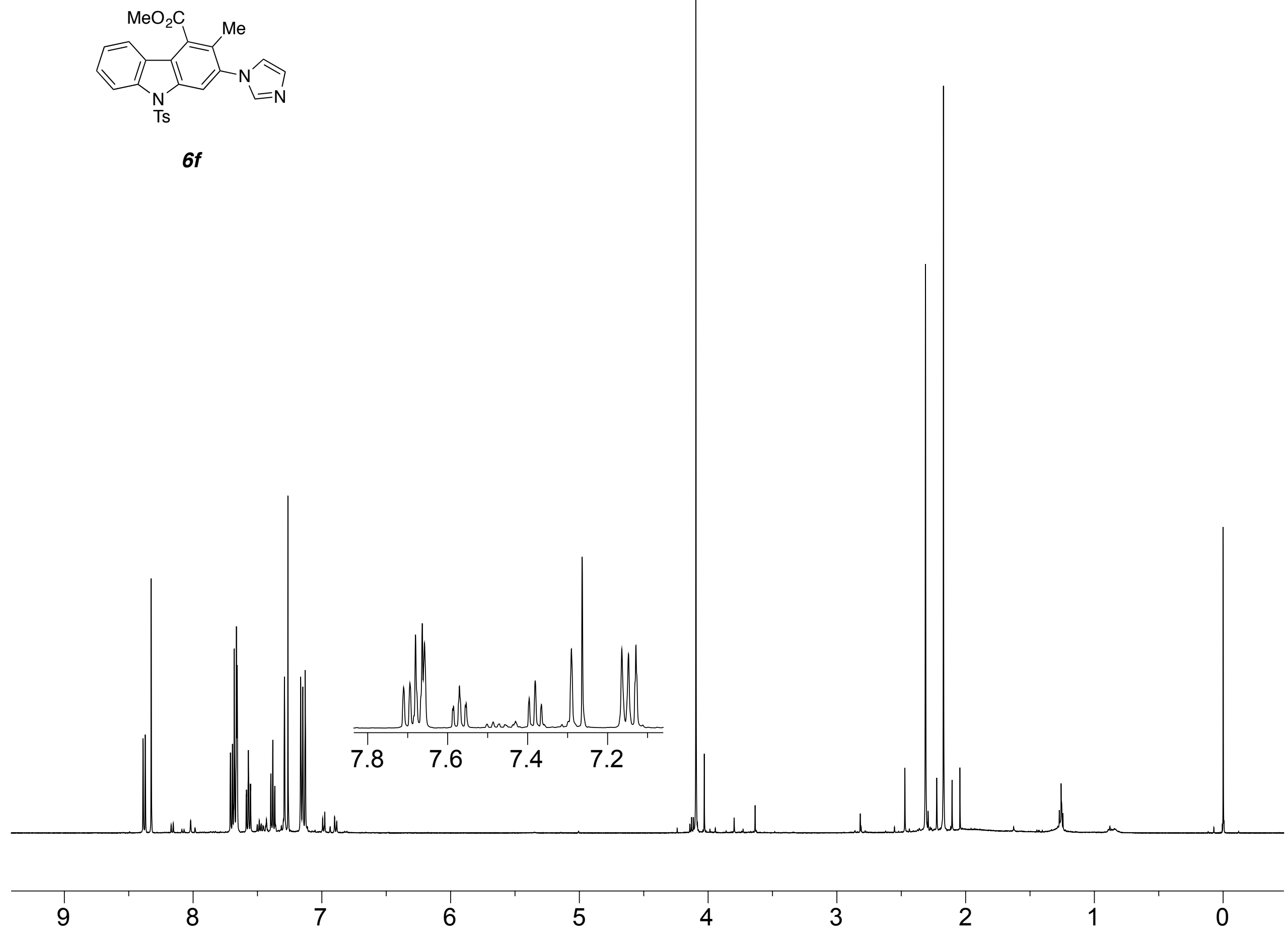


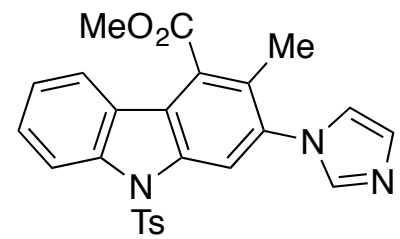

$6 f$

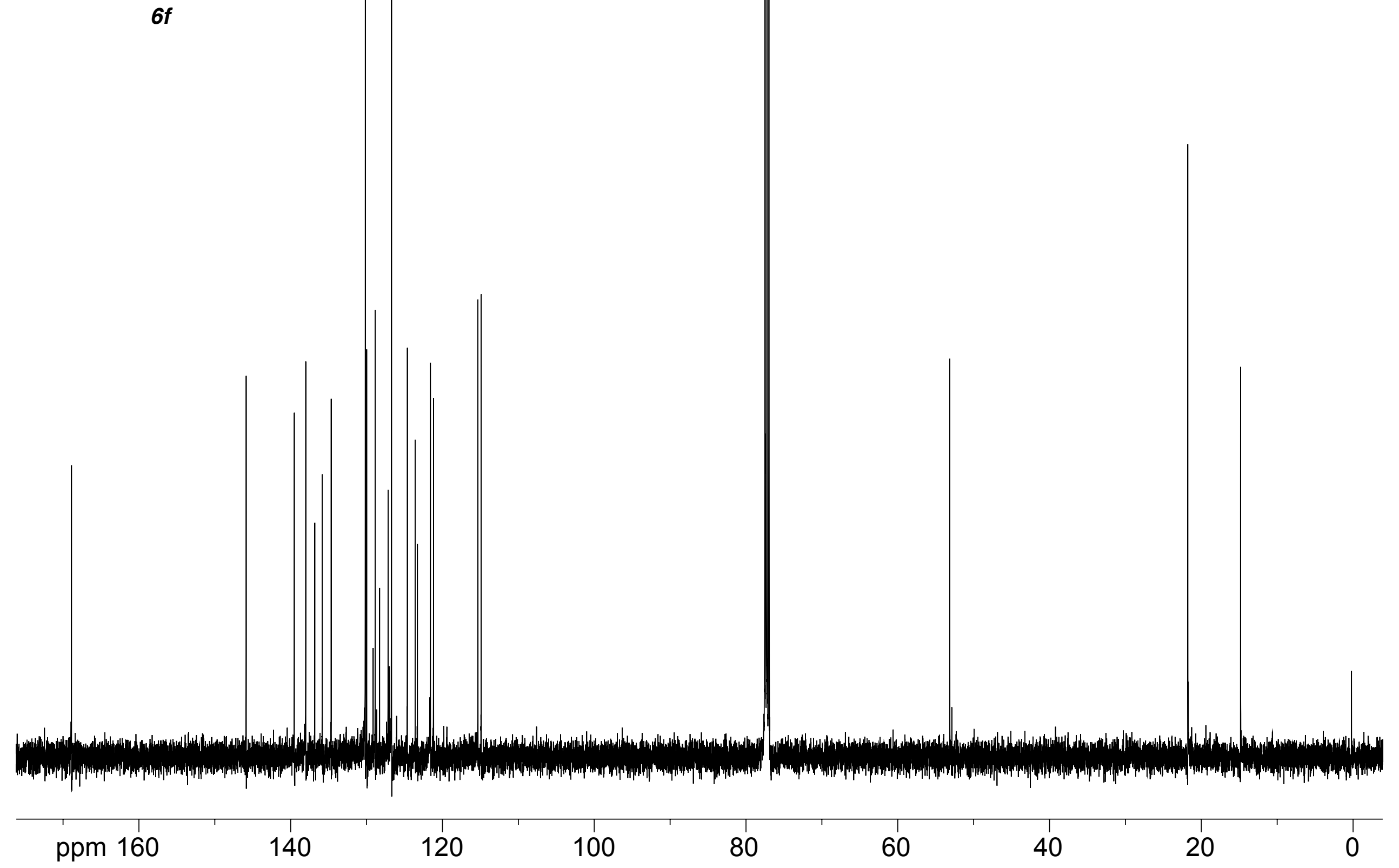



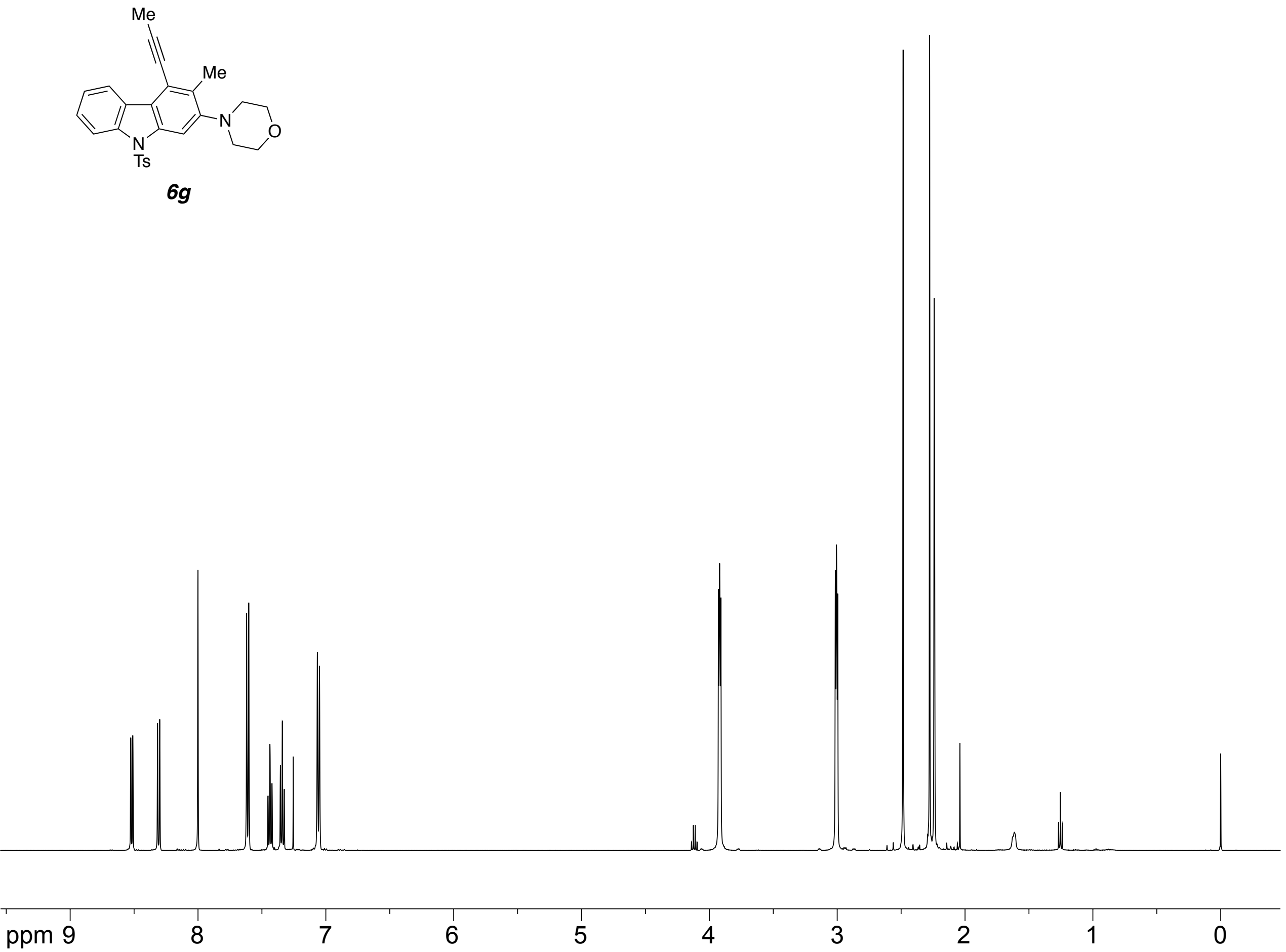

8

7

6

5

3

2

1 

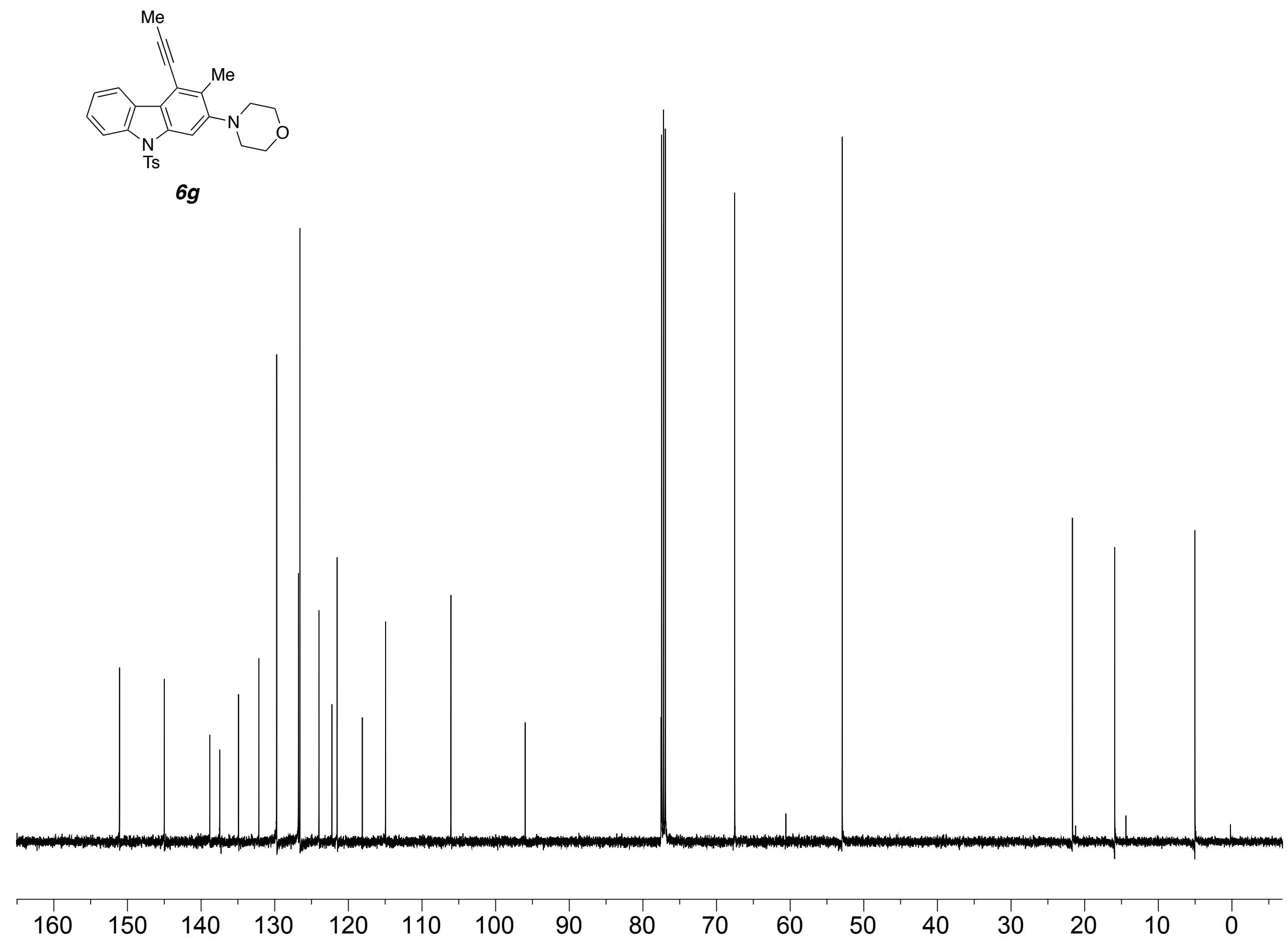


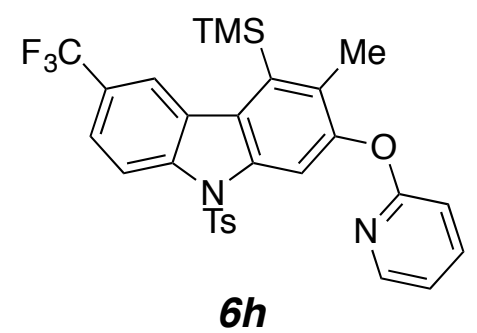

$6 h$

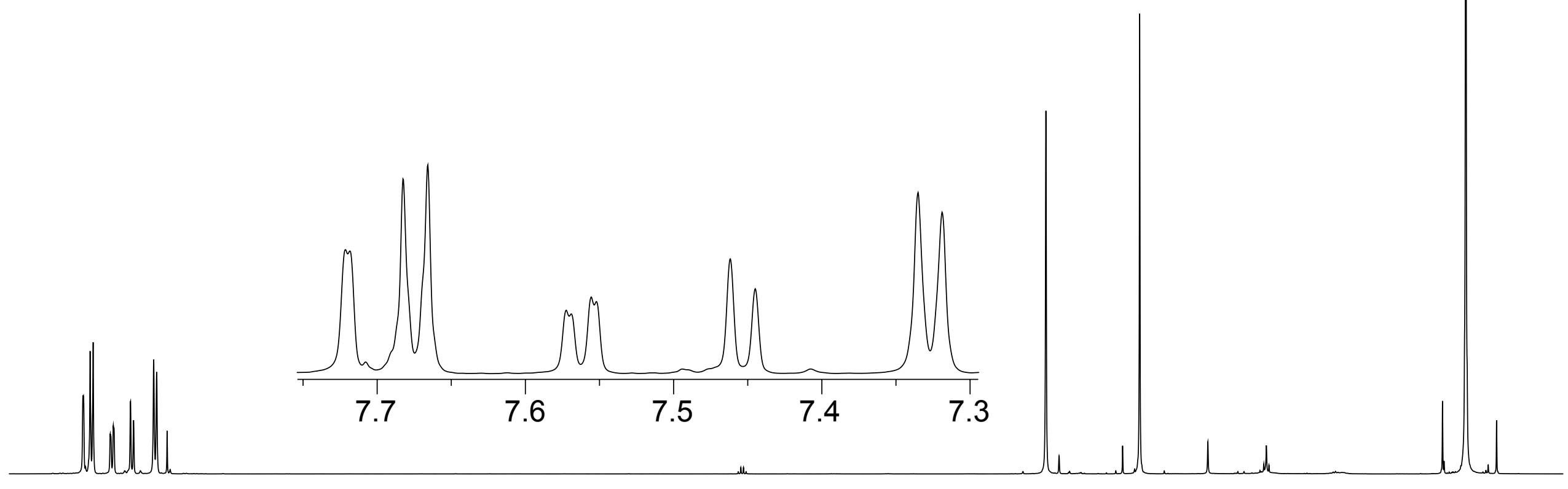

\begin{tabular}{l|l|l|l|l|l|l|l|l|}
\hline & 6 & 6 & 5 & 4 & 3 & 2 & 1 & 0
\end{tabular}



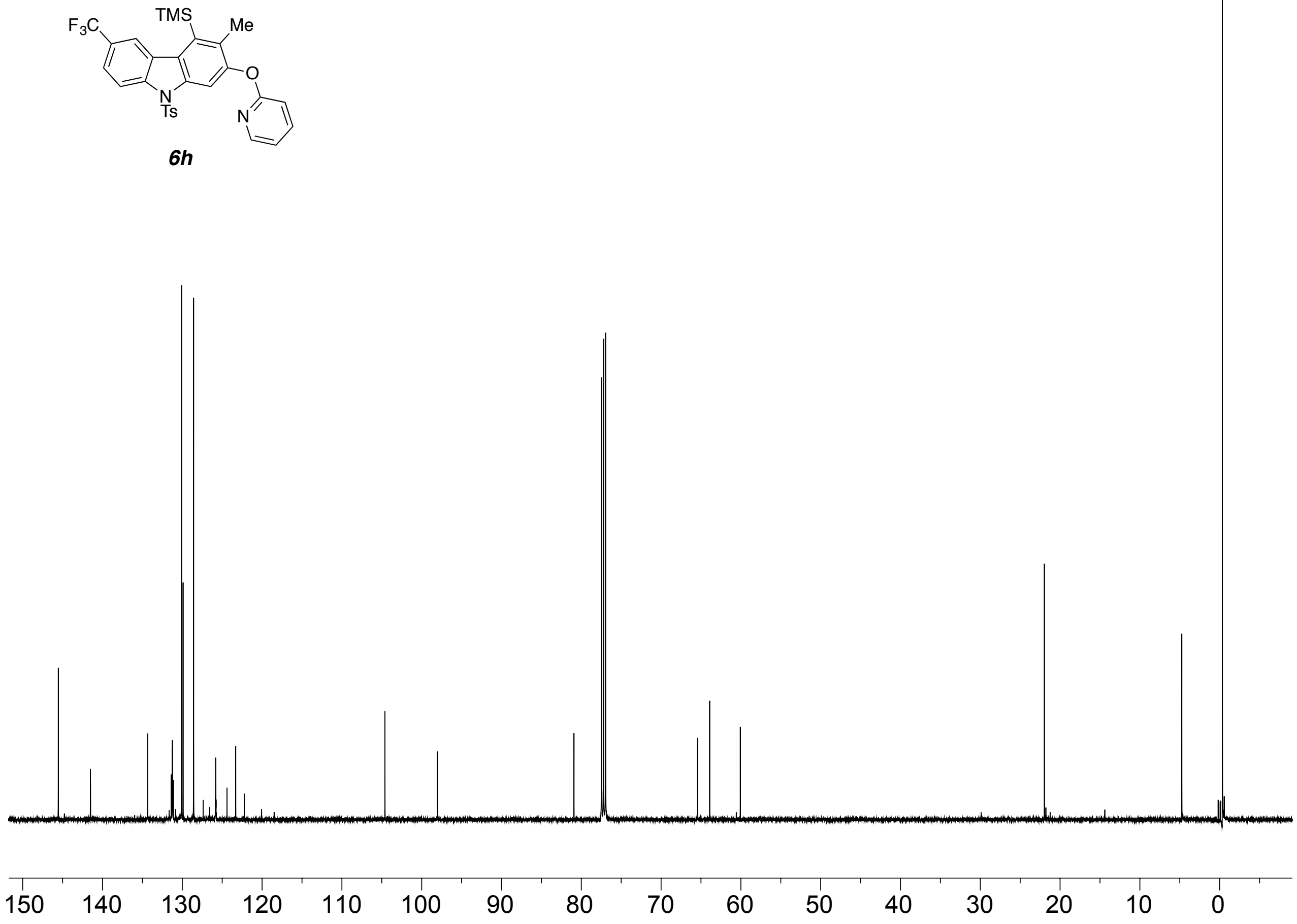

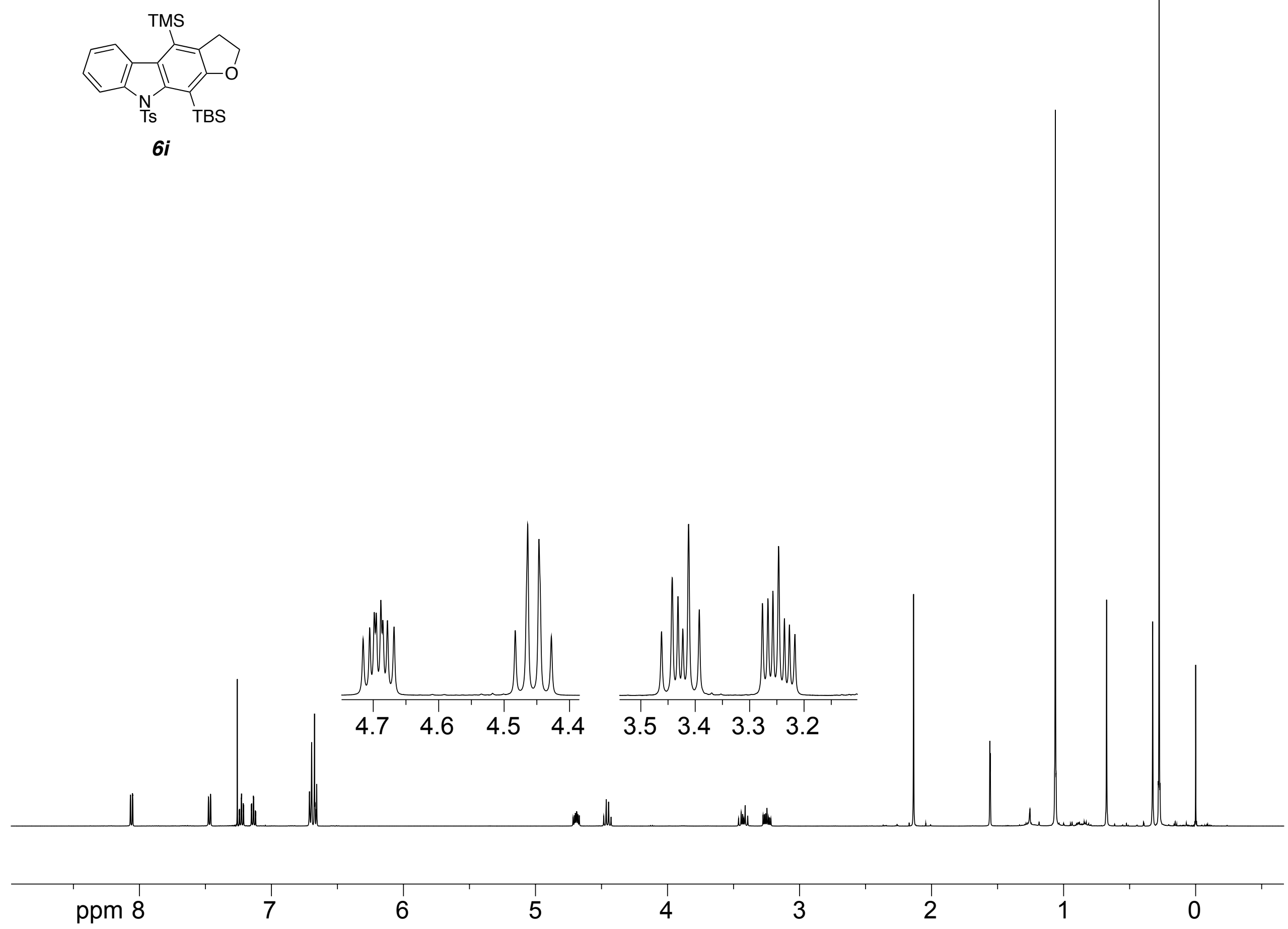

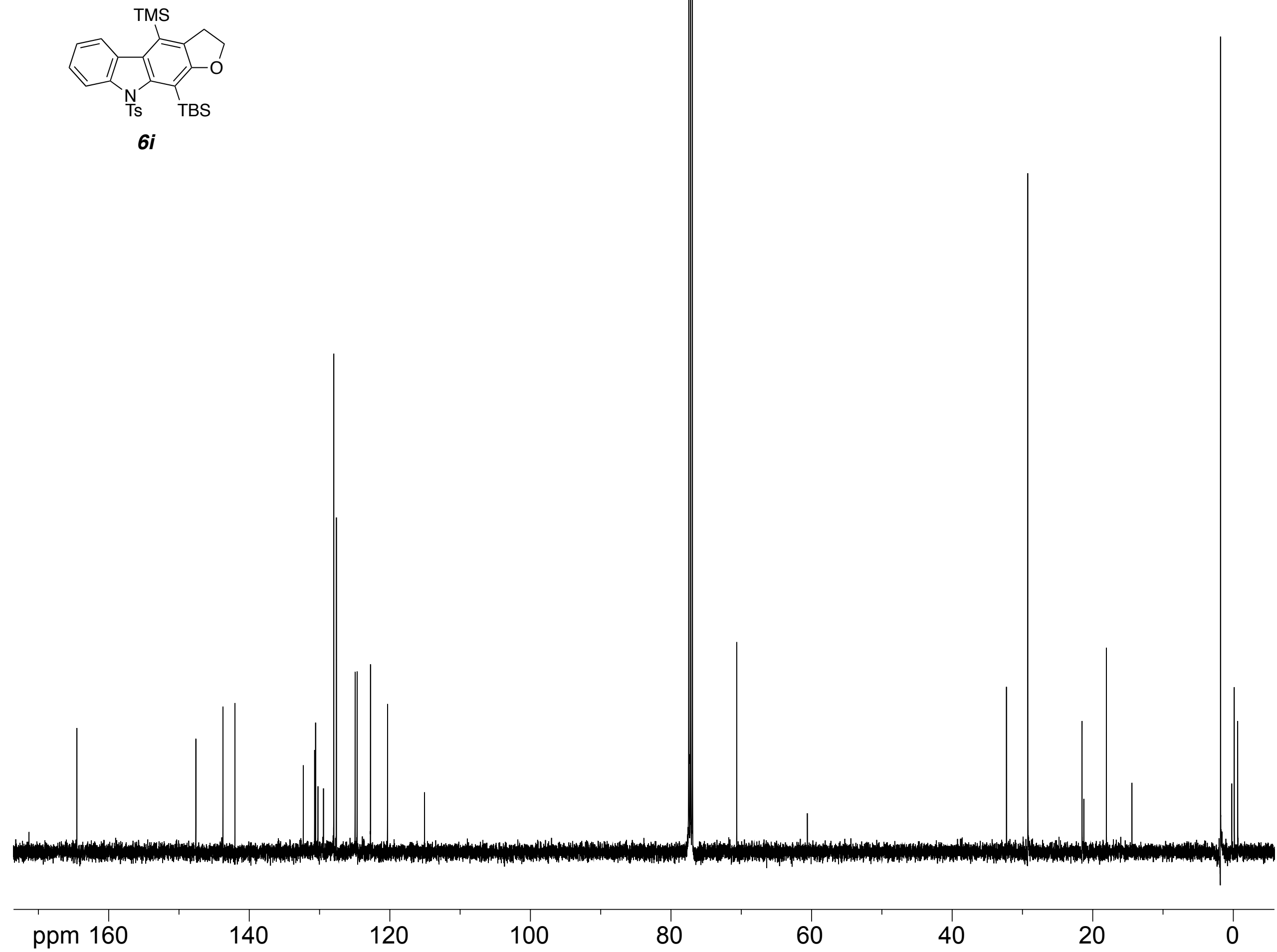

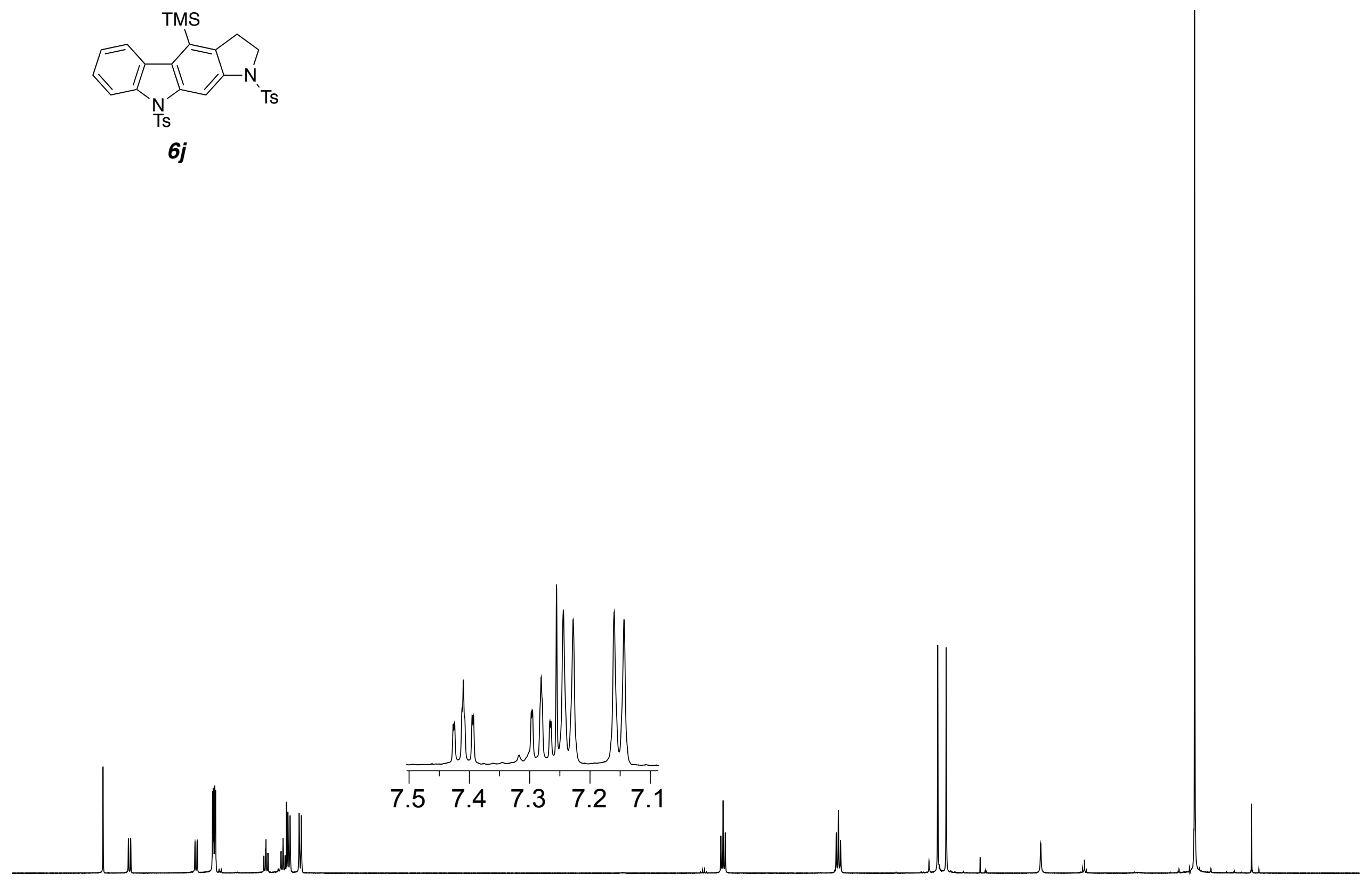

\begin{tabular}{l|ll|l|l|l|l|l|l|l|l|l|l|}
\hline & 1 & 5 & 6 & 5 & 4 & 3 & 2 & 1 & 0 & 1
\end{tabular}



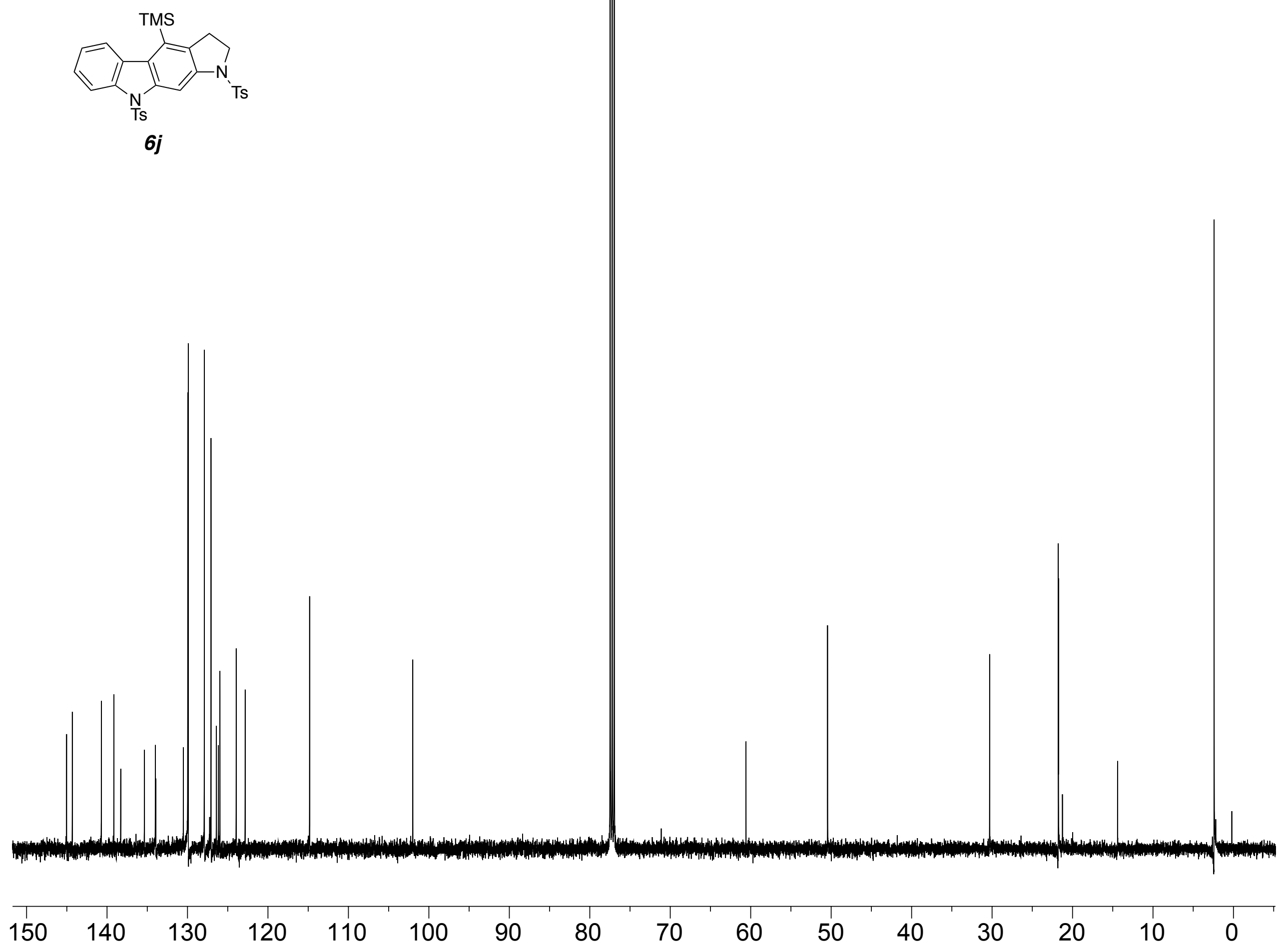

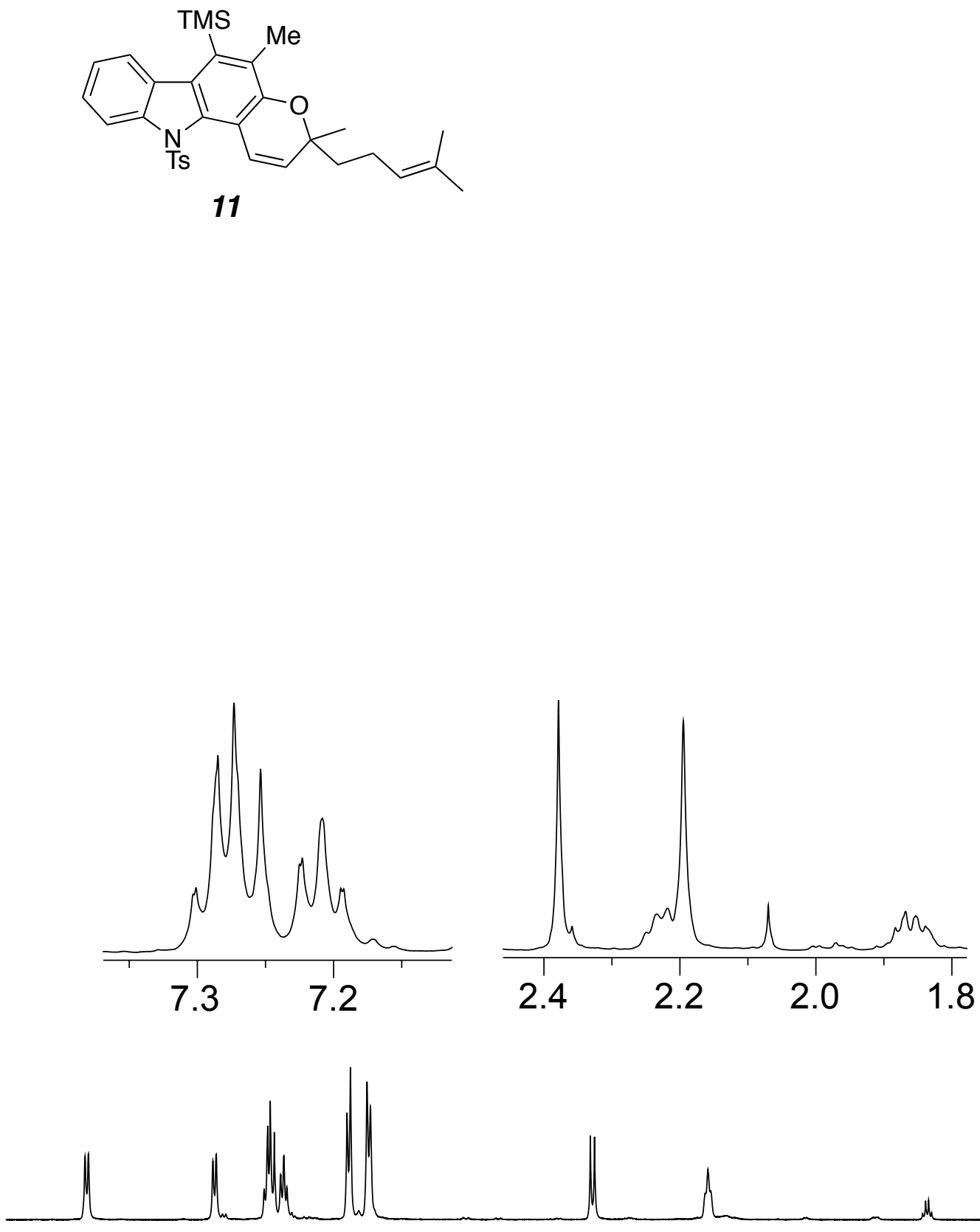
Supporting Information

Carbazole

Page S76 of S82
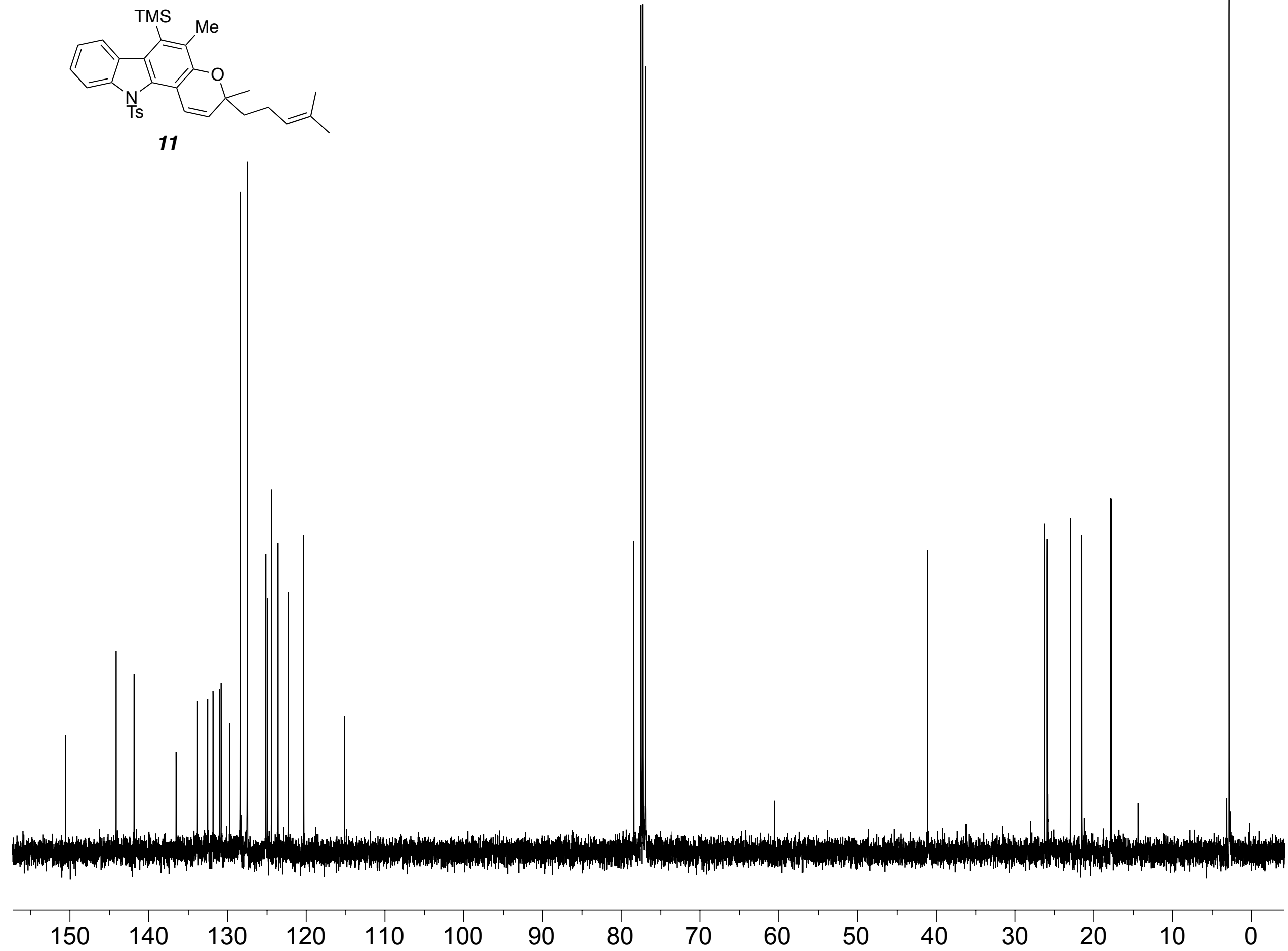

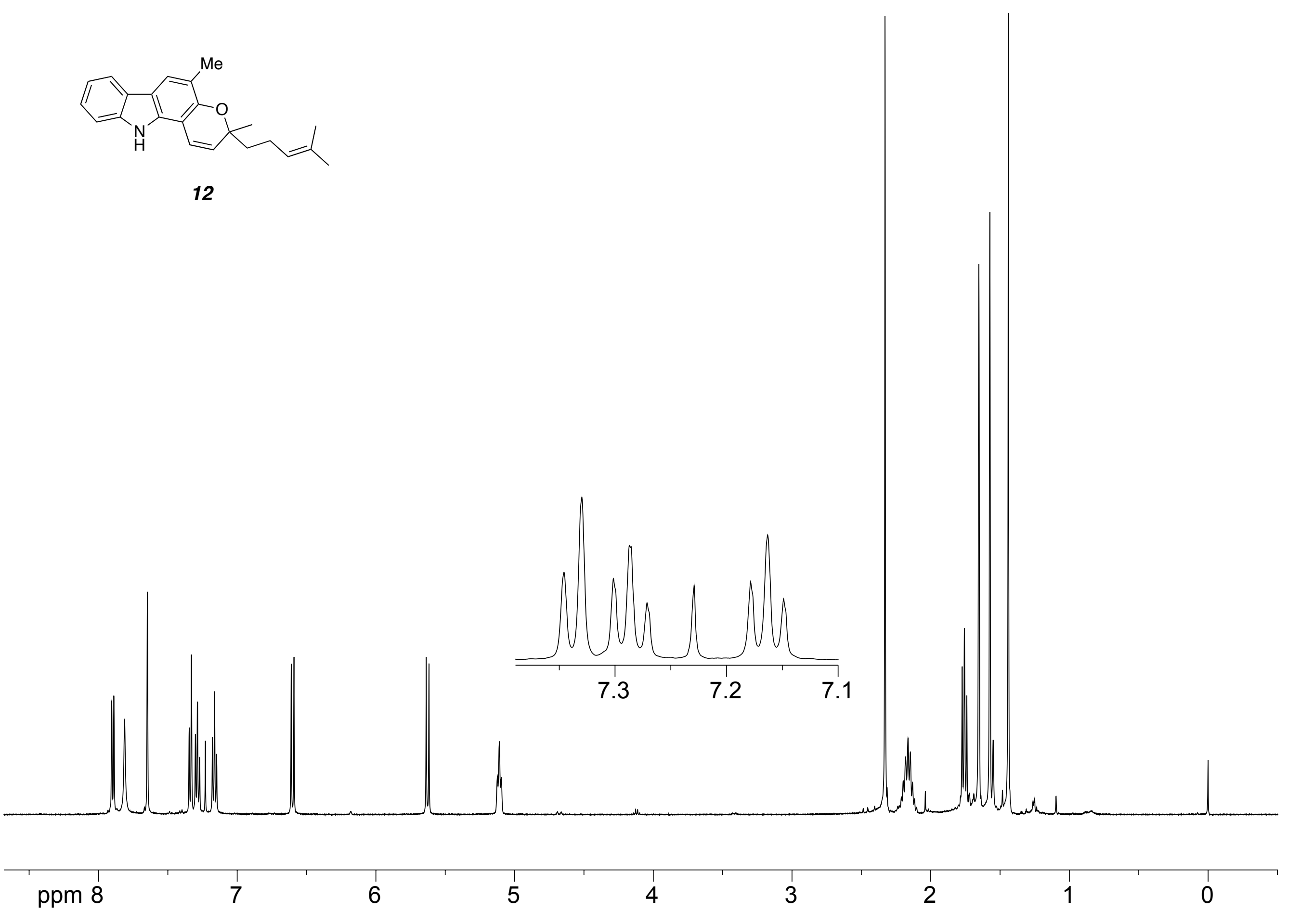
Supporting Information

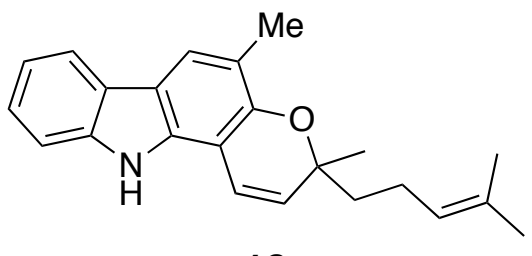

12

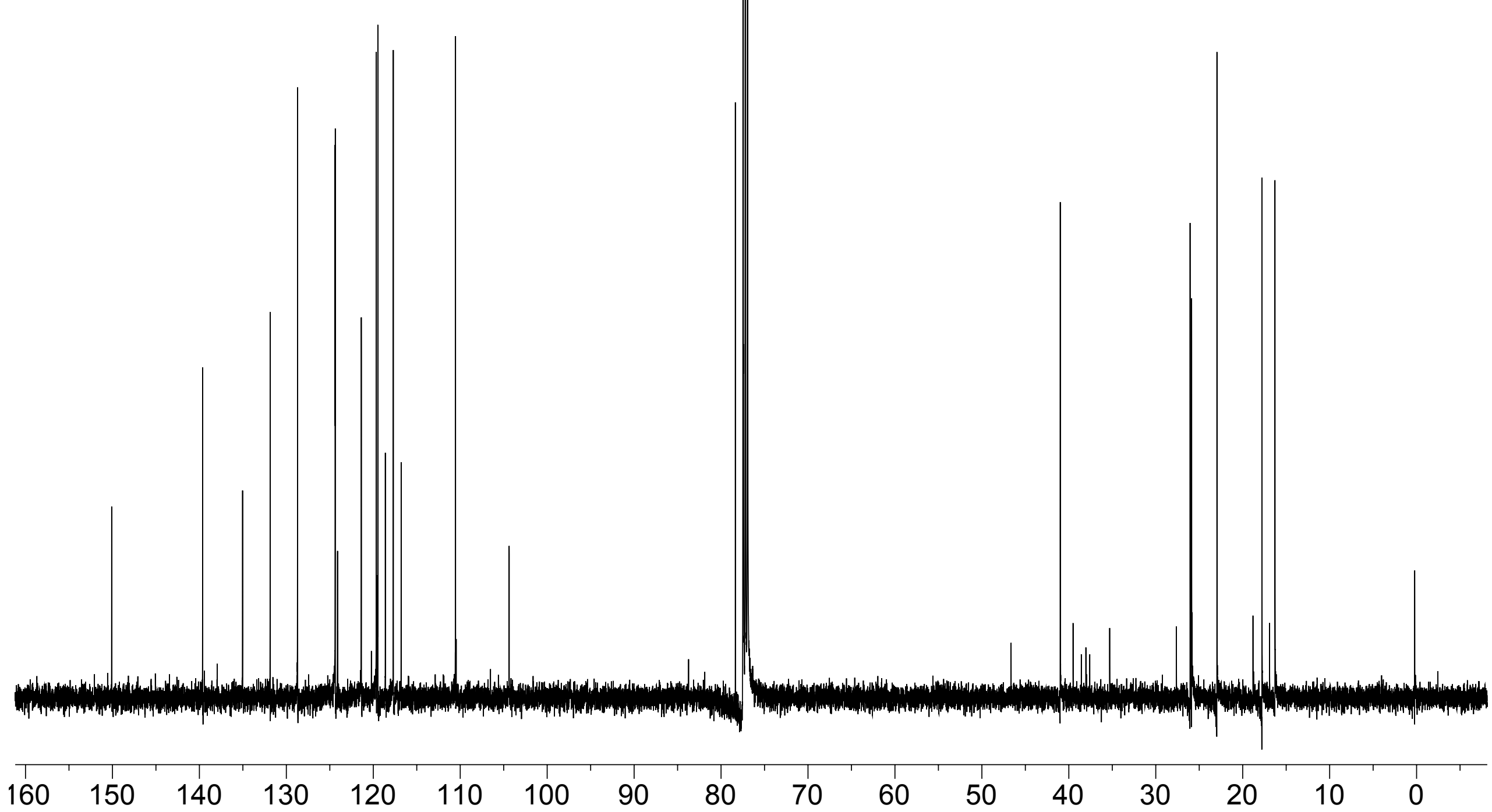




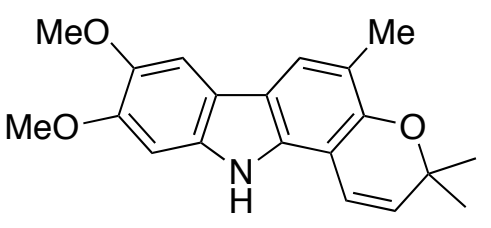

19

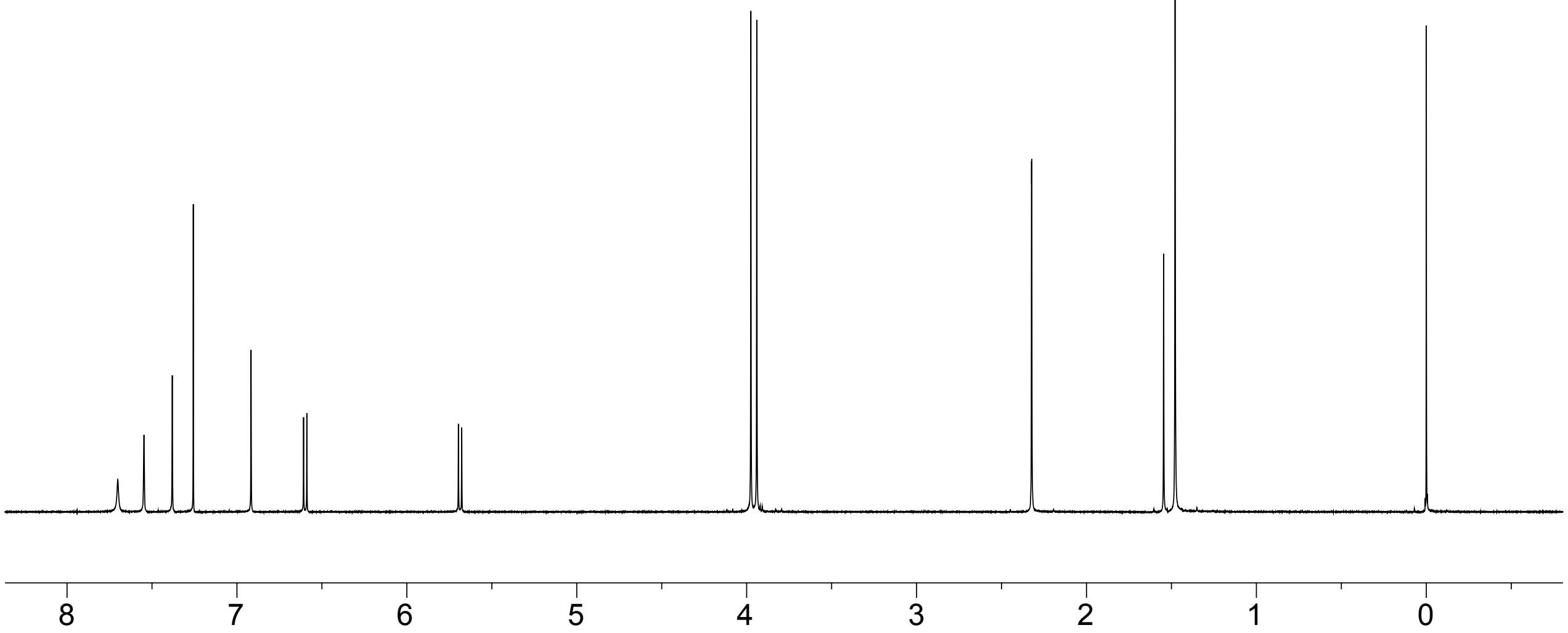




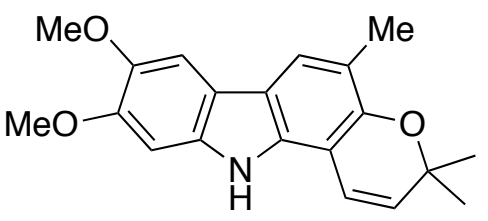

19

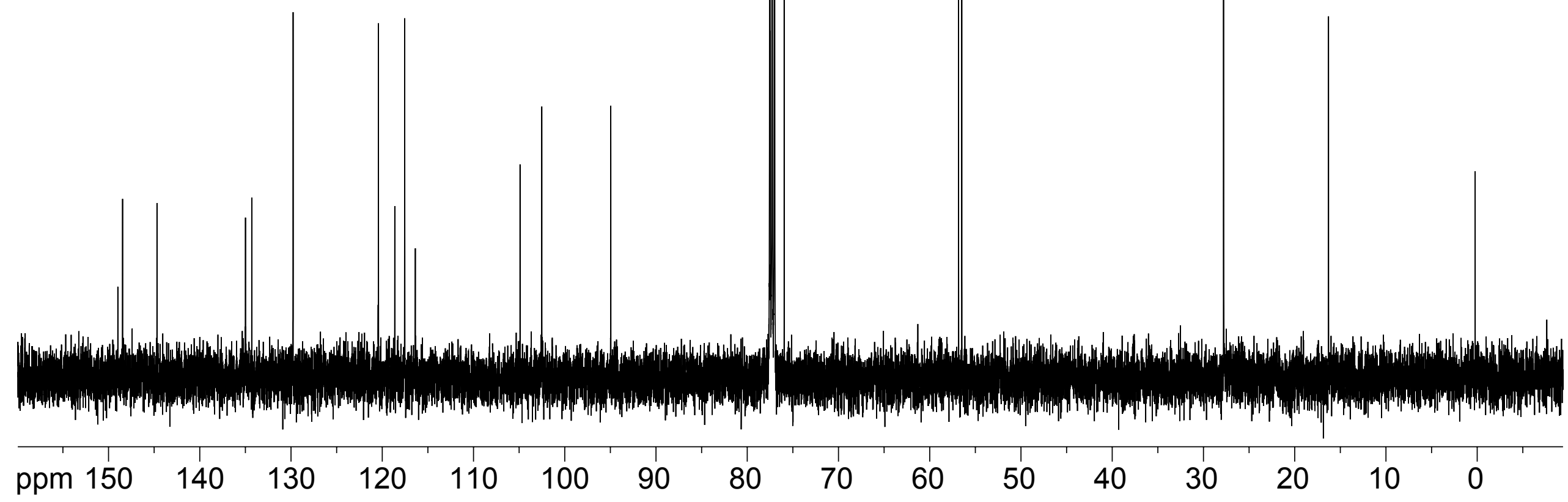



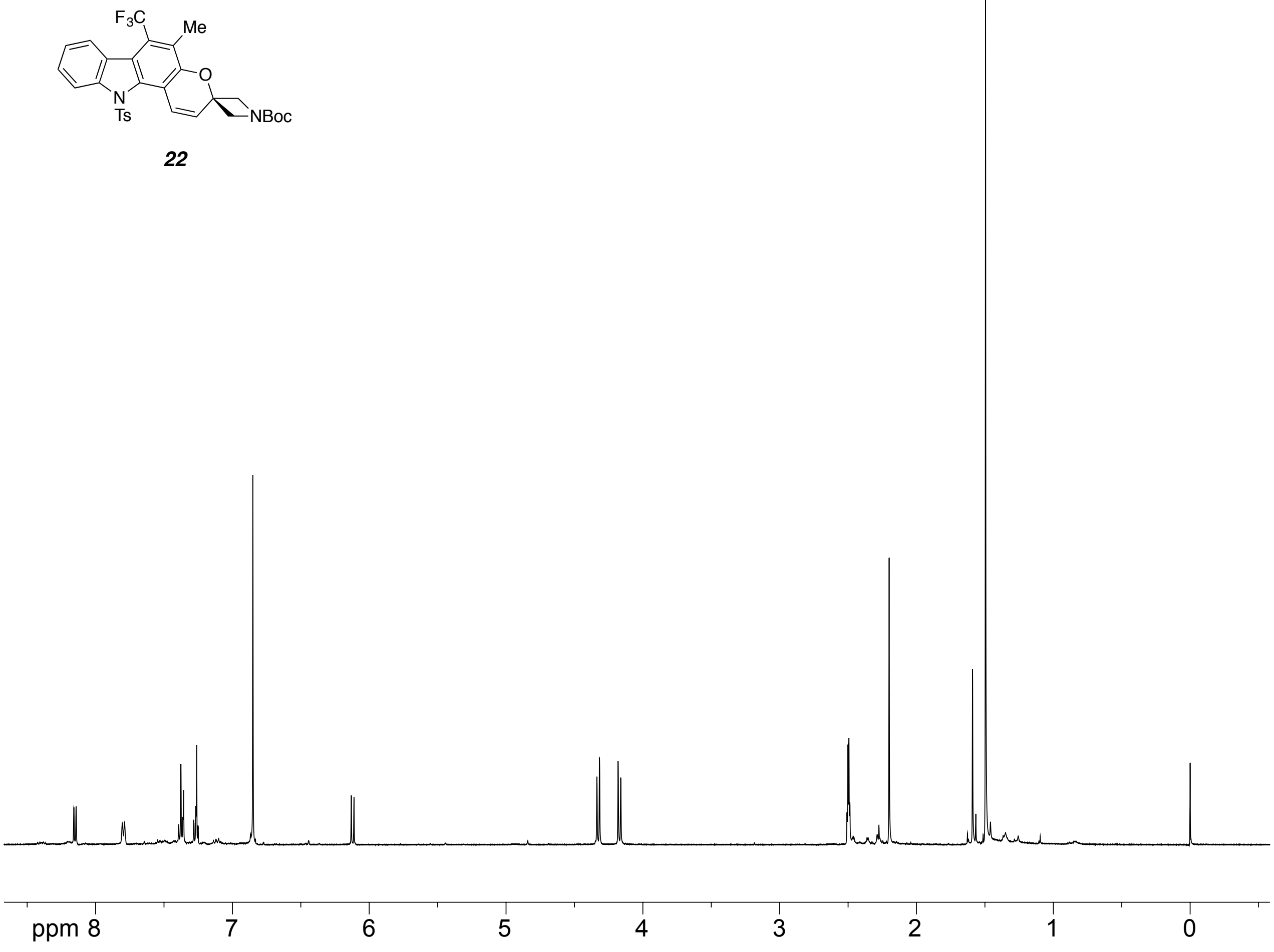


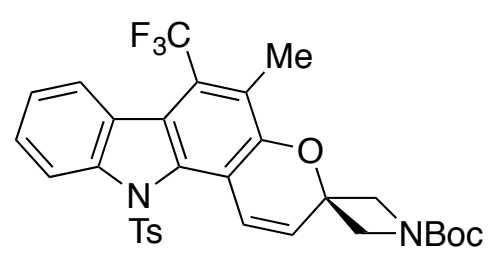

22

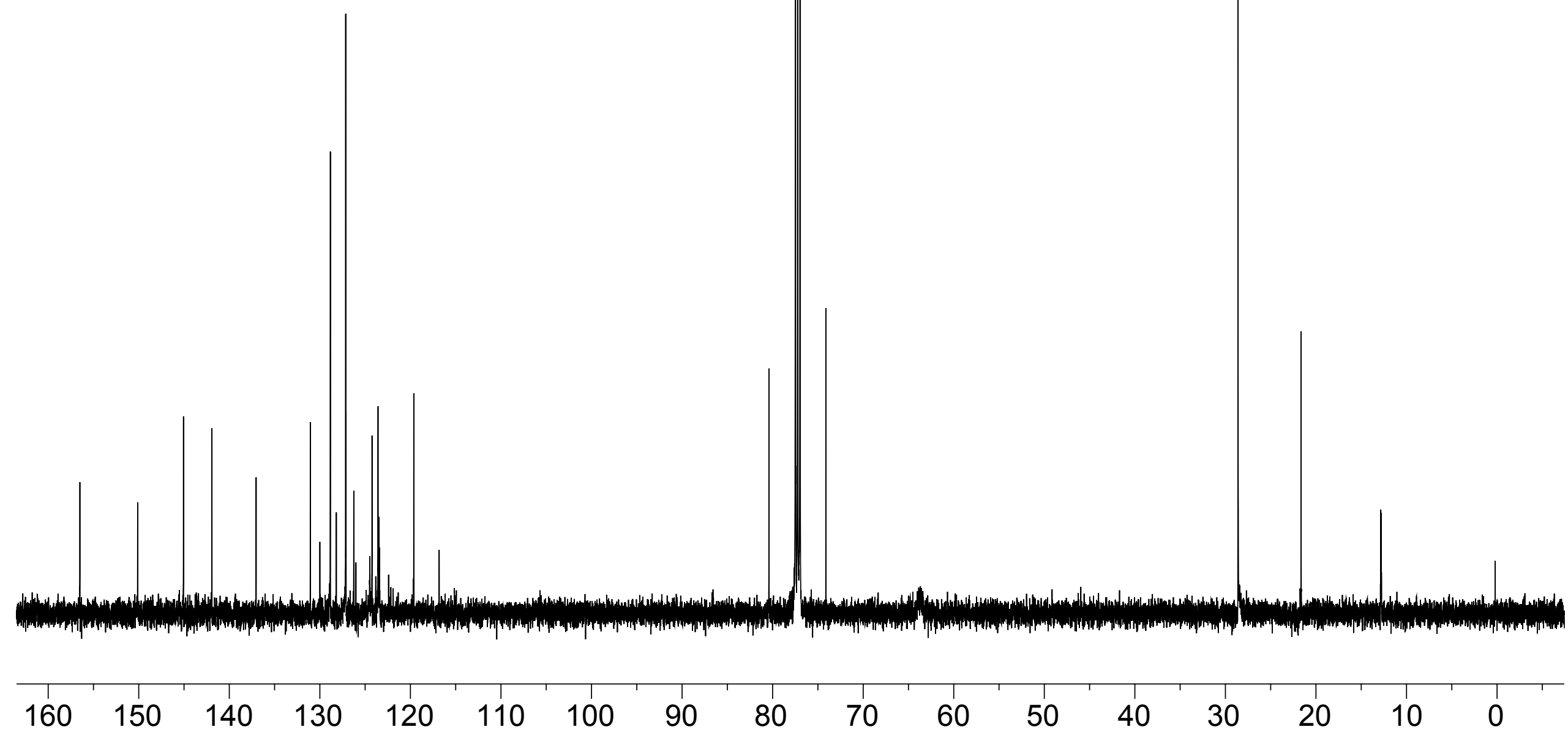

